Portland State University

PDXScholar

8-10-1992

\title{
Minimization of Exclusive Sum of Products Expressions for Multiple-Valued Input Incompletely Specified Functions
}

Ning Song

Portland State University

Follow this and additional works at: https://pdxscholar.library.pdx.edu/open_access_etds

Part of the Electrical and Computer Engineering Commons Let us know how access to this document benefits you.

Recommended Citation

Song, Ning, "Minimization of Exclusive Sum of Products Expressions for Multiple-Valued Input Incompletely Specified Functions" (1992). Dissertations and Theses. Paper 4684.

https://doi.org/10.15760/etd.6568

This Thesis is brought to you for free and open access. It has been accepted for inclusion in Dissertations and Theses by an authorized administrator of PDXScholar. Please contact us if we can make this document more accessible: pdxscholar@pdx.edu. 
AN ABSTRACT OF THE THESIS OF Ning Song for the Master of Science in Electrical and Computer Engineering presented August 10, 1992.

Title: Minimization of Exclusive Sum of Products Expressions for Multiple-Valued Input Incompletely Specified Functions

APPROVED BY THE MEMBERS OF THE THESIS COMMITTEE:

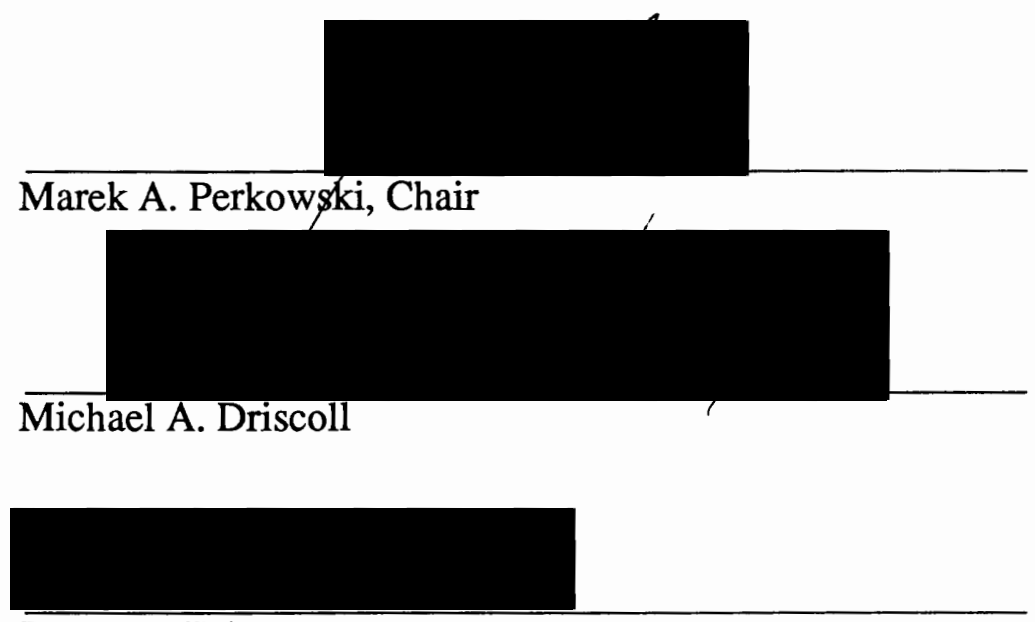

James L. Hein

In recent years, there is an increased interest in the design of logic circuits which use EXOR gates. Particular interest is in the minimization of arbitrary Exclusive Sums Of Products (ESOPs). Functions realized by such circuits can have fewer gates, fewer connections, and take up less area in VLSI and especially, FPGA realizations. They are also easily testable.

So far, the ESOPs are not as popular as their Sum of Products (SOP) counterparts. One of the main reasons it that the problem of the minimization of ESOP circuits was 
traditionally an extremely difficult one. Since exact solutions can be practically found only for functions with not more than 5 variables the interest is in approximate solutions. Two approaches to generate sub optimal solutions can be found in the literature. One approach is to minimize sub-families of ESOPs. Another approach is to minimize ESOPs using heuristic algorithms. The method we introduced in this thesis belongs to the second approach, which normally generates better results than the first approach.

In the second appraoch, two general methods are used. One method is to minimize the coefficients of Reed-Muller forms. Another method is to perform a set of cube operations iteratively on a given ESOP. So far, this method has acchieved better results than other methods.

In this method (we call it cube operation approach), the quality of the results depends on the quality of the cube operations. Different cube operations have been invented in the past a few years. All these cube operations can be applied only when some conditions are satisfied. This is due to the limitatins of the operations. These limitations reduce the opportunity to get a high quality solution and reduce the efficiency of the algorithm as well. The efferts of removing these limitatins led to the invention of our new cube operation, exorlink, which is introduced in this thesis. Exorlink can be applied on any two cubes in the array without condition. All the existing cube operations in this approach are included in it. So this is the most general operation in this approach.

Another key issue in the cube operation approach is the efficiency of the algorithm. Existing algorithms perform all possible cube operations, and give litter guide to select the operations. Our new algorithm selectively performs some of the possible operations. Experimental results show that this algorithm is more efficient than existing ones. New algorirms to minimize multiple output functions and especially incompletely specified ESOPs are also presented. The algorithms are included in the program EXORCISM-MV-2, which is a new version of EXORCISM-MV. 
EXORCISM-MV-2 was tested on many benchmark functions and compared to the results from literature. The program in most cases gives the same or better solutions on binary and 4-valued completely specified functions. More importantly, it is able to efficiently minimize arbitrary-valued and incompletely specified functions, while the programs from literature are either for completely specified functions, or for binary variables. Additionally, as in Espresso, the number of variables in our program is unlimited and the only constraint is the number of input cubes that are read, so very large functions can be minimized.

Based on our new cube operation and new algorithms, in the thesis, we present a solution to a problem that has not yet been practically solved in the literature: efficient minimization of arbitrary ESOP expressions for multiple-output multiple-valued input incompletely specified functions. 
MINIMIZATION OF EXCLUSIVE SUM OF PRODUCTS EXPRESSIONS FOR MULTIPLE-VALUED INPUT INCOMPLETELY SPECIFIED FUNCTIONS

by

NING SONG

A thesis submitted for the partial fulfillment of the requirements for the degree of

\author{
MASTER OF SCIENCE \\ in \\ ELECTRICAL AND COMPUTER ENGINEERING
}

Portland State University

1993 


\section{TO THE OFFICE OF GRADUATE STUDIES:}

The members of the Committee approve the thesis of Ning Song presented August 10, 1992.
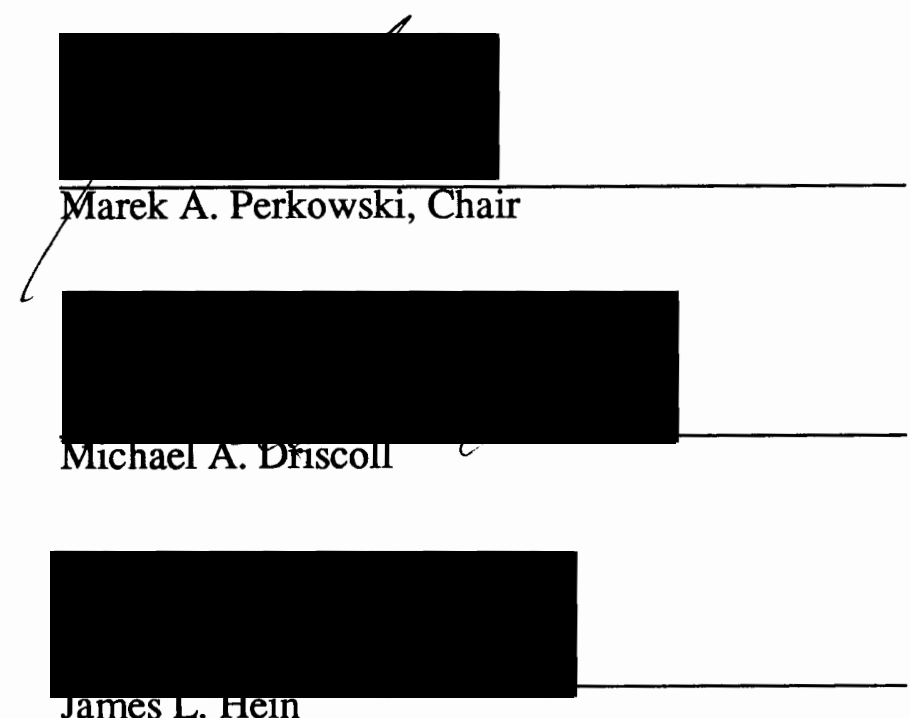

APPROVED:

Rolf Schaumann, Chalrman, Department of Electrical Engineering

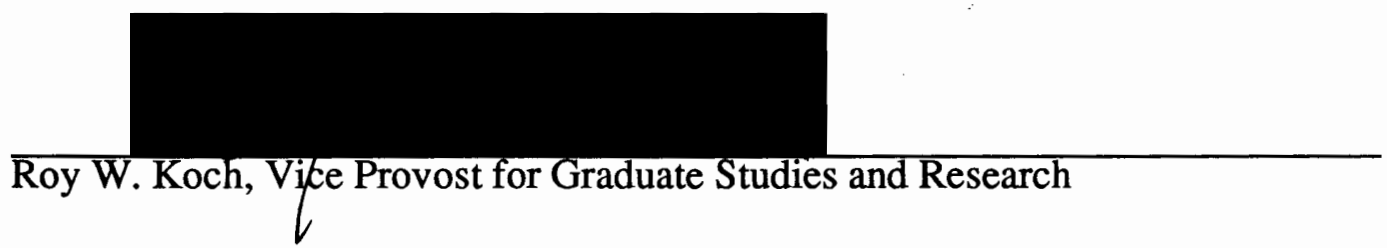




\section{ACKNOWLEDGMENT}

I would like to thank Dr. Marek A. Perkowski, my advisor, who introduced me to the area of Design Automation, guided me, and encouraged me in my research.

I would also like to thank Dr. Michael A. Driscoll, Dr. James L. Hein and Dr. W. Robert Daasch for their valuable comments which helped to improve this thesis. 
TABLE OF CONTENTS

PAGE

ACKNOWLEDGMENT $\ldots \ldots \ldots \ldots \ldots \ldots \ldots \ldots \ldots \ldots \ldots \ldots$ iii

LIST OF TABLES $\ldots \ldots \ldots \ldots \ldots \ldots \ldots \ldots \ldots \ldots \ldots \ldots \ldots \ldots \ldots \ldots \ldots \ldots$

LIST OF FIGURES $\ldots \ldots \ldots \ldots \ldots \ldots \ldots \ldots \ldots \ldots \ldots \ldots \ldots \ldots \ldots \ldots \ldots \ldots$ viii

LIST OF SPECIAL SYMBOLS $\ldots \ldots \ldots \ldots \ldots \ldots \ldots \ldots \ldots \ldots \ldots$ xi

CHAPTER

INTRODUCTION . . . . . . . . . . . . . . . .

II

BASIC CONCEPTS AND DEFINITIONS . . . . . . . . 6

II.1 Sets . . . . . . . . . . . . . 6

II.2 Functions . . . . . . . . . . . . . . . . . . $\quad 8$

II.2.1 Multiple Valued Functions

II.2.2 Cubical Representation

II.2.3 Incompletely Specified Functions

II.2.4 Multiple Output Functions

II. 3 Operations ........................ 25

II.3.1 Set Theoretic Operations

II.3.2 Cube Operations

III

MULTIPLE-VALUED INPUT EXCLUSIVE SUMS

OF PRODUCTS MINIMIZATION . . . . . . . . . . . .

III.1 The Cost Functions ................. 31

III.2 The Properties of the ESOP . . . . . . . . . . . 32

III.3 Basic Ideas to Minimize the ESOP . . . . . . . . . 33

III.3.1 Removing Two Equal cubes

III.3.2 Combining Two Cubes which Differ in One Variable

III.3.3 Reshaping Two Cubes which Differ by 2 
III.3.4 Increasing the Number of Cubes

III.4 The Operations Used in Exorcism . . . . . . . . .

III.4.1 Primary Xlinking

III.4.2 Secondary Xlinking

III.4.3 Unlinking

III.5 The Operations Used in EXMIN . . . . . . . . 40

IV

THE MULTIPLE-VALUED EXORLINKING

IV.1 The Formula ................... 45

IV.2 Difference 1 Exorlinking ............ 50

IV.2.1 Difference 1 Distance 1 Exorlinking IV.2.2 Difference 1 Distance 0 Exorlinking

IV.3 Difference 2 Exorlinking $\ldots \ldots \ldots \ldots \ldots \ldots \ldots$

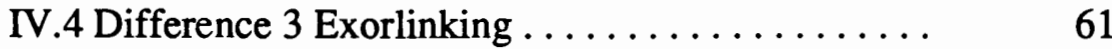

IV.5 Summary .................. 62

ALGORITHM OF EXORCISM-MV-2 PROGRAM . . . . 64

V.1 The Algorithm of EXORCISM ........... 64

V.2 The Algorithm of EXMIN ............. 64

V.3 The New Algorithm ................ 66

V.4 Minimization of Multiple Output Functions . . . . $\quad 70$

V.5 Minimization of Incompletely Specified

Functions ........................ 73

V.6 The Algorithm of EXORCISM-MV-2 ....... 81

VI

EVALUATION OF RESULTS OF EXORCISM-MV-2

85

VII

CONCLUSION ..................... 


\section{LIST OF TABLES}

TABLE

PAGE

I

An Example of Binary Input Binary Output Function . . . . . . .

II

An Example of Multiple-Valued Input Binary Output Function

III

Examples of Incompletely Specified Functions . . . . . . . .

An Example of Multiple-Valued Input Binary Output

Incompletely Specified Function

VII

Converting a Multiple Output Function to a Single

Output Function

Experimental Results of Functions with 1 Bit and 2 Bit

Decoders

XVI Comparison of EXORCISM-MV-2 with EXMIN-2 
XVII Comparison of EXORCISM-MV-2 with EXMIN-2

for the Same Execution Time $\ldots \ldots \ldots \ldots \ldots \ldots \ldots \ldots$

XVIII Comparison of Our Results with ESPRESSO . . . . . . . . . 92

XVII Comparison of Minimization of ON-Cubes with Minimization of ON- and DC-Cubes ................... 92 


\section{LIST OF FIGURES}

FIGURE

PAGE

1. ON- and OFF-arrays of function $f \ldots \ldots \ldots \ldots \ldots \ldots \ldots \ldots$

2. Karnaugh map for a binary input function of 3 variables ...... 13

3. Map for a multiple-valued input function of 2 variables ..... 13

4. Karnaugh map for binary input cubes .............. 17

5. Map for multiple-valued input cubes $\ldots \ldots \ldots \ldots \ldots \ldots . \ldots$

6. Map for a multiple-valued input incompletely specified function $\ldots \ldots \ldots \ldots \ldots \ldots \ldots \ldots \ldots \ldots \ldots \ldots \ldots \ldots, 21$

7. ON-, OFF-, and DC-array of an incompletely specified function $\ldots \ldots \ldots \ldots \ldots \ldots \ldots \ldots \ldots \ldots \ldots \ldots \ldots, 21$

8. $\quad$ ESOPs for a multiple-valued input incompletely specified function $\ldots \ldots \ldots \ldots \ldots \ldots \ldots \ldots \ldots \ldots \ldots \ldots$

9. Map for a multiple output function $\ldots \ldots \ldots \ldots \ldots \ldots \ldots \ldots$

10. Map and circuit for a multiple output function ........... 26

11. Example of a disjoint sharp operation ............. 30

12. Combining two cubes into one cube $\ldots \ldots \ldots \ldots \ldots \ldots . \ldots \ldots$

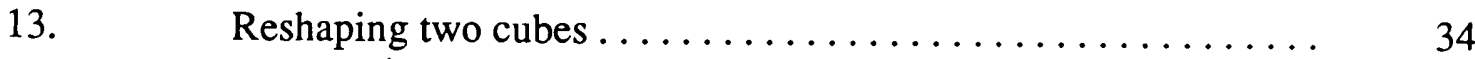

14. Procedure of distance 1 primary xlinking $\ldots \ldots \ldots \ldots \ldots . \ldots 36$

15. Procedure of distance 2 primary xlinking ............. 37

16. Procedure of distance 1 secondary xlinking ............ 39

17. Procedure of distance 2 secondary xlinking $\ldots \ldots \ldots \ldots \ldots .40$

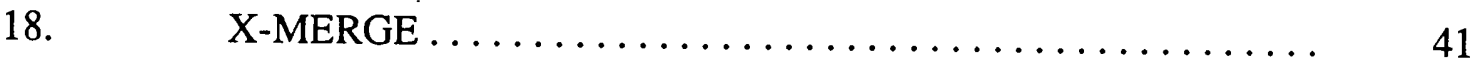


An example of exorlinking two terms .

26. An example of exorlinking two cubes .

Difference 1 distance 1 exorlinking

28. An example of difference 1 distance 0 exorlinking $\left(S_{i} \supset R_{i}\right) \ldots$

Difference 2 exorlinking $\left(S_{i} \cap R_{i}=\varnothing, S_{j} \backslash R_{j}\right) \ldots \ldots \ldots \ldots$

38. An example of ESOP minimization by difference 2 exorlinking

39. An example of ESOP minimization by difference 3 exorlinking

41. An example of performing all possible difference 2 operations .

43. Conditionally performing difference 2 exorlinking ......... 
45. Example of minimizing an incompletely specified function ....

46. $\quad$ Linking DC-cubes $\ldots \ldots \ldots \ldots \ldots \ldots \ldots \ldots \ldots \ldots \ldots$

47. The position of DC-cubes $\ldots \ldots \ldots \ldots \ldots \ldots \ldots \ldots \ldots$

48. The size of DC-cubes $\ldots \ldots \ldots \ldots \ldots \ldots \ldots \ldots \ldots \ldots$

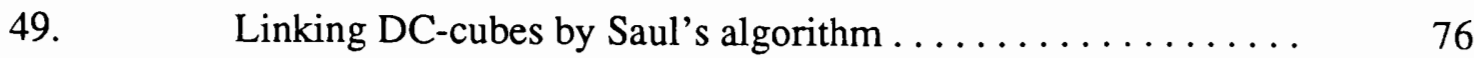

50. An ON-cube is equal to a DC-cube $\ldots \ldots \ldots \ldots \ldots \ldots \ldots$

51. An ON-cube is contained by a DC-cube $\ldots \ldots \ldots \ldots \ldots \ldots$

52. Minimization of an incompletely specified function . . . . . . 80

53. Scatter plot of number of terms versus execution time $\ldots \ldots \ldots 8$

54. Scatter plot of number of variables versus execution time . . . $\quad 89$ 


\section{LIST OF SPECIAL SYMBOLS}

Page number refers to the page on which the symbol is defined or first used.

SYMBOL

PAGE

Set Relations

$\epsilon$

Is a member of

6

$\subseteq$

Is a subset of

6

$\subset$

Is a proper subset of 7

$\varnothing$

Empty set

$\times$

Cartesian product

Two sets are overlapping

Is not a subset of

Set Operations

u

Union of two sets

$\cap$

Intersection of two sets

Difference of two sets

$\oplus$

Exclusive-OR of two sets

Complement of a set

Logic Operations

$\oplus$

Exclusive-OR operator

Cube Operations

$\cup$

Supercube of two cubes 


\section{CHAPTER I}

\section{INTRODUCTION}

In recent years, there is an increased interest in the design of logic circuits which use EXOR gates [Hell 88, Sasa 90b]. Particular interest is in the minimization of arbitrary Exclusive Sums Of Products (ESOPs) and their various canonical sub-families such as Consistent Generalized Reed-Muller canonical forms (CGRMs) [Lui 92, Sara 92], Kronecker Reed-Muller forms [Gree 91, Gill 91], Canonical Restricted Mixed Polarity forms (CRMPs) [Csan 92], and other. Functions realized by such circuits can have fewer gates, fewer connections, and take up less area in VLSI and especially, FPGA realizations. They are also easily testable [Fuji 86, Prad 87]. It was shown, both theoretically and experimentally [Sasa 90b, Sasa 91a, Sasa 91b, Sara 92, Salm 89] that ESOPs have on average smaller numbers of terms for both "worst case" and "average" Boolean functions. It was also shown that ESOPs and all their sub-families have their counterparts in logic with multiple-valued inputs: Multiple-valued Input ESOPs (MIESOPs) [Perk 89, Sasa 90a], Multiple-valued Input Generalized Reed-Muller forms (MIGRMs) [Scha 91], Multiple-valued Input Kronecker Reed-Muller forms (MIKRMs) [Scha 92], Multiplevalued Input Generalized Reed-Muller Trees (MIGRMTs) [Perk 91], and others [Perk 92]. Logic with multiple-valued inputs (mv logic, for short) generalizes the classical Boolean logic and finds many important applications in logic design [Sasa 78, Sasa 81, Sasa 87, Rude 85]. MIESOPs are never worse than ESOPs, and they were shown to be superior on several classes of functions [Sasa 90b, Sasa 91a, Sasa 91b]. Minimized ESOP and MIESOP expressions are the starting points to technology mapping for new EXOR-based low granularity FPGAs such as CLi 6000 series from Concurrent Logic 
Inc. [CLi 91]. ESOPs are also a starting point to ESOP factorization procedures which produce multi-level AND-EXOR circuits [Saul 91]. Finally, ESOPs are used as the internal function representation in GATEMAP [Salm 89] and POLO [Perk 92] design automation systems.

So far, the ESOPs are not as popular as their Sum of Products (SOP) counterparts. One of the main reasons is that the problem of the minimization of ESOP circuits was traditionally an extremely difficult one. Papakonstantinou [Papa 79] gave an exact algorithm for 4 input functions. The algorithm from [Perk 90] has theoretically no limits on the number of variables but 4 is its practical limit. Since exact solutions can be practically found only for functions with not more than 5 variables the interest is in approximate solutions. Two approaches to generate sub optimal solutions can be found in the literature. One approach is to minimize sub-families of ESOPs. Another approach is to minimize ESOPs using heuristic algorithms. Efficient programs for sub-families of ESOPs were given in [Bess 83, Csan 92, Scha 91]. Heuristic computer programs have been presented in [Bess 91, Flei 83, Flei 87, Hell 88, Perk 89, Robi 82, Saul 90]. In these programs, two general methods are used. One method is to minimize the coefficients of Reed-Muller forms [Wu 82, Bess 83, Robi 82]. Another method is to perform a set of cube operations iteratively on minterms or disjoint cubes. Fleisher, et. al. [Flei 87] presented an algorithm which starts from positive Reed-Muller forms and performs three cube operations iteratively. The paper [Hell 88] introduced a new cube operation crosslink, and presented an algorithm based on this new operations. The algorithm from [Hell 88] was next improved in [Perk 89], and also extended for the case of logic with multiple-valued inputs. The unlink operation has also been added. The unlink operation was efficiently implemented in [Saul 90]. A few more cube operations were also included in an independent realization by Sasao [Sasa 90a], which is the only other author that published on the most general ESOP minimization algorithms for the 
multiple-valued input logic. Contrary to [Sasa 90a], however, the program described in [Perk 89] assumed that the number of truth values for each variable can be different. Although the theory presented by Sasao in [Sasa 90a] is general, the EXMIN algorithm implemented by him, for efficiency reasons, accepts only 2 -valued and 4 -valued variables. It also can not deal with incompletely specified functions.

In this thesis, we present a solution to a problem that has not yet been practically solved in the literature: efficient minimization of arbitrary ESOP expressions for multiple-output multiple-valued input incompletely specified functions. This thesis describes an approximate method that yields especially good results for the minimization of strongly unspecified multi-output logic functions with multiple-valued inputs and binary-valued outputs. Our program, EXORCISM-MV-2 is a new version of EXORCISM-MV presented in [Perk 89]. The algorithm currently used in EXORCISMMV-2 was created after much experimentation with previous variants. A new cube operation - exorlinking is also introduced to support the new algorithm.

Our method and program are applicable to any type of mu'tiple-valued input functions, and each input variable can have an arbitrary number of logic values. For simplicity, 4-valued logic will be presented in most of the multiple valued logic examples. This variant finds, among others, applications in the minimization of PLAs in which pairs of inputs are implemented via 2-by-4 decoders ( 2 inputs, 4 outputs).

AND/EXOR circuits with various kinds of function generators on inputs to the AND plane are used to realize the MIESOP expressions minimized here. Such circuits can have smaller complexity than both the circuits which implement mixed-polarity ESOP expressions [Perk 89], AND/XOR-Fields [Froe 91] , PLAs with decoders [Sasa 84], and networks with two variable function generators [Sasa 86]. The most important advantage of the presented approach is that it can produce FPGA circuits that are superior (in terms of both speed and area) to those obtained using other methods for the logic 
with multiple-valued inputs and are also very easily testable. There are several ways to realize MIESOP circuits in modern technologies: XOR PLAs (XPLAs, AND/XORFields), standard cells, optical and Josephson junction AND/EXOR PLAs, and especially various types of FPGAs (like those from Xilinx, Actel, CLi, Algotronix, and Texas Instruments) [FPGA 92]. A particularly well-suited FPGA is the recently announced CLi 6000 series from Concurrent Logic Inc. [CLi 91], since one of the most efficient uses of its basic logic cell is the 3-input AND/EXOR function [Wu 92].

In Chapter II, the basic concepts and definitions related to this thesis are presented. The main concepts in this chapter are the multiple-valued input binary output incompletely specified functions, the exclusive-OR sum-of-products expressions (ESOPs), and the corresponding cube notations.

In Chapter III, we first introduce the basic ideas of ESOP minimization. Two major programs for ESOP minimization, EXORCISM and EXMIN are then presented. The operations used in these two programs are discussed in detail with examples.

Our new cube operation, exorlinking, is presented in Chapter IV. Exorlinking generalizes all cube operations such as xlinking, unlinking, or $\mathrm{X}$-merge introduced in EXORCISM [Hell 88, Perk 89] and EXMIN [Sasa 90a]. Contrary to the operations in EXORCISM and EXMIN, which can be applied in certain conditions, we proved in this chapter that exorlinking can be applied in any two cubes in arbitrary distance. The procedure of the exorlinking operation is discussed, different examples are given, and comparisons with operations in EXORCISM and EXMIN are shown.

In Chapter V, we first introduce the algorithms used in EXORCISM and EXMIN. Then our new algorithm used in EXORCISM-MV-2 is discussed. The major advantage of our new algorithm is that it gives priority to those difference 2 operations which will directly reduce the number of cubes in the array. By this way, our program can achieve better results in shorter time as compared to the former algorithms. Our algorithms to 
handle multiple output functions and incompletely specified functions are also discussed in this chapter.

Chapter VI shows the experimental results. Then in the last chapter, Chapter VII, we give the conclusion. The "man page" about how to use our program is presented in the appendix with examples. 


\section{CHAPTER II}

\section{BASIC CONCEPTS AND DEFINITIONS}

In this chapter, we give some basic definitions and discuss some basic concepts. These concepts and definitions are necessary for the following chapters. More detailed discussion on these topics can be found in [Stol 79, Grat 79, Brow 90].

\section{II.1. SETS}

A set is a collection of objects; the objects in the collection are called elements of the set. Here we consider only finite sets, i.e., sets possessing a finite number of elements. We write

$$
x \in P
$$

to denote that $x$ is an element of a set named $P$.

If all the elements of set $P$ are also elements of set $Q$, then we say that $P$ is included in (or is a subset of) $Q$ and we represent the relation between $P$ and $Q$ by writing

$$
P \subseteq Q
$$

If $P \subseteq Q$ and $Q \subseteq P$, we write

$$
P=Q
$$

$P=Q$ indicates that sets $P$ and $Q$ contain exactly the same elements.

If $P=Q$ does not hold, we write

$$
P \neq Q \text {. }
$$

$P \neq Q$ indicates that sets $P$ and $Q$ do not contain exactly the same elements. 
If $P$ is a subset of $Q$, and $Q$ contains at least one element that does not belong to $P$, then $P$ is called a proper subset of $Q$, and we write

$$
P \subset Q
$$

to indicate the relation between $P$ and $Q$. By definition, $P \subset Q$ means $P \subseteq Q$ and $P \neq Q$

The empty set is denoted by $\varnothing$, and is the set comprising no elements at all. The empty set is included in every set, i.e.,

$$
\varnothing \subseteq P
$$

for any set $P$.

We write $P(k)$ to indicate that $P$ contains $k$ elements. For instance, $P(2)$ denotes that $P$ contains two elements.

The Cartesian (direct, cross) product of sets $P$ and $Q$, written $P \times Q$, is the set defined by

$$
P \times Q=\{(p, q) \mid p \in P \text { and } q \in Q\}
$$

Thus

$$
\{p, q\} \times\{p, q, r\}=\{(p, p),(p, q),(p, r),(q, p),(q, q),(q, r)\}
$$

We write $P^{n}(k)$ to signify the $n$-fold Cartesian product of $P(k)$ with itself, i.e.,

$$
P^{n}(k)=P(k) \times P(k) \times \cdots \times P(k) .
$$

In more general cases, we write $P^{n}$ to denote $P_{1}\left(k_{1}\right) \times P_{2}\left(k_{2}\right) \times \cdots \times P_{n}\left(k_{n}\right)$, where $P_{1}\left(k_{1}\right), P_{2}\left(k_{2}\right), \ldots, P_{n}\left(k_{n}\right)$ are sets, and they may or may not contain the same elements. If $P_{1}\left(k_{1}\right)=P_{2}\left(k_{2}\right)=\ldots=P_{n}\left(k_{n}\right)$, then $P^{n}(k)=P^{n}$. So, $P^{n}(k)$ is a special case of $P^{n}$. 


\section{II.2. FUNCTIONS}

\section{II.2.1. Multiple Valued Functions}

A completely specified function (function for short) $f$ from set $P^{n}$ to set $Q$ is a subset of $P^{n} \times Q$, such that for each $p \in P^{n}$, there is a $q \in Q$, and $(p, q) \in f$. We write

$$
f: P^{n} \rightarrow Q
$$

to denote that $f$ takes elements from the sets $P_{1}, P_{2}, \ldots, P_{n}$ to yield elements in the set $Q$.

Given a function $f: P^{n} \rightarrow Q, P^{n}$ is called the domain of the function; $Q$ is called the co-domain of the function. If all of the sets $P_{i}(i=1, \ldots, n)$ in the domain $P$ contain two elements, the function is a binary input function. For example, $f: P_{1}(2) \times P_{2}(2) \rightarrow Q$ is a binary input function. If some of the sets in the domain contain more than two elements, the function is a multiple-valued input function. For example, $f: P_{1}(4) \times P_{2}(4) \rightarrow Q$ and $f: P_{1}(2) \times P_{2}(4) \rightarrow Q$ are multiple-valued input functions. If the co-domain of the function contains two elements only, it is a binary output function. A binary output function is also called a switching function. If the co-domain contains more than two elements, it is a multiple-valued output function. Only the binary output functions will be discussed in this thesis.

A symbol that may represent any one of the elements of set $P_{i}$ or $Q$ is called a variable.

Example II.1: Given a set $P_{i}=\{0,1,2,3\}$ which contains 4 elements. If $X$ can assume values $0,1,2$, or 3 , then $X$ is a 4 -valued variable.

A variable corresponding to a set in the domain is called an input variable. A variable corresponding to a set in the co-domain is called an output variable.

If the domain of a function is $P^{n}$, we call the function an $n$-input function. If $n=$ 1 , the function is a single input function. If the domain of the function is $P^{n}(2)$, it is a $n$ - 
binary-input function. On the other hand, if one or more sets in the domain have more than two elements, it is a multiple-valued multiple-input function. If the domain is $P^{n}(k)$, its radix is fixed. If the domain is $P_{1}\left(k_{1}\right) \times P_{2}\left(k_{2}\right) \times, \ldots, \times P_{n}\left(k_{n}\right)$, and $\exists(i, j)$ such that $k_{i} \neq k_{j}$, the radix is mixed.

If $p_{i}$ is a positive integer, $P_{i}=\left\{0,1, \ldots, p_{i}-1\right\}$ is a set in the domain, and $X_{i}$ is a variable corresponding to $P_{i}$, then for any subset $S_{i} \subseteq P_{i}, X_{i} S_{i}$ is a literal of variable $X_{i}$ representing a function such that

$$
X_{i} S_{i}=\left\{\begin{array}{ll}
1 & \text { if } X_{i} \in S_{i} \\
0 & \text { if } X_{i} \notin S_{i}
\end{array} .\right.
$$

A literal is a function that maps from domain $P_{i}$ to co-domain $Q(2)$. The variable $X_{i}$ can take one of the values of 0 through $p_{i}-1$. We write $X=0$ to indicate that $X$ takes value of $0, X=1$ to indicate that $X$ takes value of 1 , etc. $S_{i}$ specifies the set of values of $X_{i}$ for which $X_{i}^{S_{i}}$ is true. For instance, for a binary logic, $P_{i}=\{0,1\}$ :

$$
\begin{aligned}
& X_{i}^{\varnothing} \text { is false for both } X_{i}=0 \text { and } X_{i}=1, \\
& X_{i}^{1} \text { is true only for } X_{i}=1, \\
& X_{i}^{0} \text { is true only for } X_{i}=0, \\
& X_{i}^{0,1} \text { is true for both } X_{i}=0 \text { and } X_{i}=1 .
\end{aligned}
$$

For a binary logic, we also write $X_{i}^{1}$ as $X_{i}$ and $X_{i}^{0}$ as $\bar{X}_{i}$. So, in the case of binary logic, a literal can be represented by a variable or its complement.

For a 4-valued logic, $p_{i}=\{0,1,2,3\}$

$$
\begin{aligned}
& X_{i}^{0,1,2,3} \text { is true for any value of } X_{i}, \\
& X_{i}^{0,2} \text { is true for } X_{i}=0 \text { or } X_{i}=2, \\
& X_{i}^{0,2} \text { is false for } X_{i}=1 \text { or } X_{i}=3 .
\end{aligned}
$$

A product of literals, $X_{1}{ }_{1}^{S_{1}} X_{2}^{S_{2}} \ldots X_{n}{ }^{S_{n}}$, is referred to as a product term (also called term or product for short). A product term that includes literals for all function 
variables $X_{1}, X_{2}, \ldots, X_{n}$ is called a full term. A sum of products is denoted as a sum-ofproducts (SOP) expression while a product of sums is called a product-of-sums (POS) expression. An Exclusive-Or of products will be called an ESOP. The name MultipleValued Input Exclusive Sum of Products Form (MIESOP for short ) will be used if we want to emphasize that the ESOP takes multiple-valued inputs.

Example II.2: Given a function $f$ as specified by Table I.

\section{TABLE I}

AN EXAMPLE OF BINARY INPUT BINARY OUTPUT FUNCTION

\begin{tabular}{lll|l}
$x$ & $y$ & $z$ & $f$ \\
\hline 0 & 0 & 0 & 0 \\
0 & 0 & 1 & 1 \\
0 & 1 & 0 & 0 \\
0 & 1 & 1 & 1 \\
1 & 0 & 0 & 0 \\
1 & 0 & 1 & 1 \\
1 & 1 & 0 & 1 \\
1 & 1 & 1 & 1
\end{tabular}

Table I is called a truth table. A truth table enumerates the values of functions for each of their possible combinations of the inputs. Each row of the truth table presents a possible input combination and an associated output value. The set of those input combinations, whose associated output values are 1 , is referred to as an $\mathrm{ON}$-array of the function $f$. The set of those input combinations, whose associated output values are 0 , is referred to as an OFF-array of the function $f$. In Table I, $x, y$, and $z$ are binary input variables, $f$ is a binary output variable. Figure 1 shows the ON- and OFF-arrays of $f$. Please note that either $\mathrm{ON}$-array or OFF-array of the function $f$ contain the same information as the truth table.

The function $f$ can also be specified by the following Boolean equation:

$$
f=\bar{x} \bar{y} z \oplus \bar{x} y z \oplus x \bar{y} z \oplus x y \bar{z} \oplus x y z .
$$




\begin{tabular}{cccccc}
\multicolumn{3}{c}{ ON-array } & \multicolumn{3}{c}{ OFF-array } \\
$x$ & $y$ & $z$ & $x$ & $y$ & $z$ \\
0 & 0 & 1 & 0 & 0 & 0 \\
0 & 1 & 1 & 0 & 1 & 0 \\
1 & 0 & 1 & 1 & 0 & 0 \\
1 & 1 & 0 & & & \\
1 & 1 & 1 & & &
\end{tabular}

Figure 1. ON- and OFF- arrays of function $f$.

where $\bar{x} \bar{y} z \oplus \bar{x} y z \oplus x \bar{y} z \oplus x y \bar{z} \oplus x y z$ is an ESOP expression. Please note that $x, y$ and $z$ in Table I are variables, while in the ESOP expression they are used as literals. Another way to write above equation is:

$$
f=x^{0} y^{0} z^{1} \oplus x^{0} y^{1} z^{1} \oplus x^{1} y^{0} z^{1} \oplus x^{1} y^{1} z^{0} \oplus x^{1} y^{1} z^{1}
$$

Example II.3: In Table II, $X$ and $Y$ are multiple-valued variables, $f$ is a binary output variable.

TABLE II

AN EXAMPLE OF MULTIPLE-VALUED INPUT BINARY OUTPUT FUNCTION

\begin{tabular}{ll|l}
$X$ & $Y$ & $f$ \\
\hline 0 & 0 & 0 \\
0 & 1 & 1 \\
0 & 2 & 0 \\
1 & 0 & 1 \\
1 & 1 & 0 \\
1 & 2 & 1 \\
2 & 0 & 1 \\
2 & 1 & 1 \\
2 & 2 & 0
\end{tabular}

This function can be represented by the following equation:

$$
f=X^{0} Y^{1} \oplus X^{1} Y^{02} \oplus X^{2} Y^{01}
$$

where $X^{0}, X^{1}, X^{2}, Y^{1}, Y^{02}$, and $Y^{01}$ are literals. 
The difference of two terms is the number of variables for which the corresponding literals have different sets of truth values. The distance of two terms is the number of variables for which the corresponding literals have disjoint sets of truth values.

Example II.4: Given three terms $T_{1}=X^{0} Y^{1}, T_{2}=X^{1} Y^{02}$, and $T_{3}=X^{1} Y^{01}$. The difference of $T_{1}$ and $T_{2}$ is 2 , because two literals have different sets of truth values:

$$
\begin{aligned}
& \text { for } X:\{0\} \neq\{1\}, \\
& \text { for } Y:\{1\} \neq\{02\}
\end{aligned}
$$

The distance of $T_{1}$ and $T_{2}$ is also 2 , because two literals have disjoint sets of truth values:

$$
\begin{aligned}
& \text { for } X:\{0\} \cap\{1\}=\varnothing, \\
& \text { for } Y:\{1\} \cap\{02\}=\varnothing .
\end{aligned}
$$

The difference of $T_{2}$ and $T_{3}$ is 1 , because only one literal has different sets of truth values:

$$
\begin{aligned}
& \text { for } X:\{1\}=\{1\}, \\
& \text { for } Y:\{02\} \neq\{01\}
\end{aligned}
$$

The distance of $T_{2}$ and $T_{3}$ is 0 , because none of the literals have disjoint sets of truth values:

$$
\begin{aligned}
& \text { for } X:\{1\} \cap\{1\} \neq \varnothing, \\
& \text { for } Y:\{02\} \cap\{01\} \neq \varnothing .
\end{aligned}
$$

We write difference $\left(T_{i}, T_{j}\right)=d$ to indicate that the difference of two terms $T_{i}$ and $T_{j}$ is $d$. Similarly, we write distance $\left(T_{i}, T_{j}\right)=d$ to indicate that the distance of $T_{i}$ and $T_{j}$ is $d$.

A map of an $n$-variable, $p$-valued input, two-valued output function consists of $p^{n}$ cells. Cells that contain a 1 will be called true minterms (1-cells) while cells that contain a 0 will be called false minterms (0-cells of the map). For a binary input function, our 
map is a Karnaugh map. For the case of multiple-valued input functions, the maps that we will use in this thesis generalize the concept of the Karnaugh maps. We will simply call each of them a map.

In Example II.2, the function has 3 binary variables. So, a corresponding Karnaugh map has $2^{3}=8$ cells. There are 5 true minterms and 3 false minterms as shown in Figure 2.

\begin{tabular}{|c|c|c|c|c|}
\hline $\mathrm{y}^{2}$ & 00 & 01 & 11 & 10 \\
\hline 0 & 0 & 1 & 1 & 0 \\
\hline 1 & 0 & 1 & 1 & 1 \\
\hline
\end{tabular}

Figure 2. Karnaugh map for a binary input function of 3 variables.

In Example II.3, the function has 2 variables, and both variables have three values. So, a corresponding map has $3^{2}=9$ cells. There are 5 true minterms and 4 false minterms as shown in Figure 3.

\begin{tabular}{|c|c|c|c|}
\hline & 0 & 1 & 2 \\
\hline 0 & 0 & 1 & 0 \\
\hline 1 & 1 & 0 & 1 \\
\hline 2 & 1 & 1 & 0 \\
\hline
\end{tabular}

Figure 3. Map for a multiple-valued input function of 2 variables. 


\section{II.2.2. Cubical Representation}

A binary vector (vector for short) is a series of symbols, where each symbol is either a 0 or a 1 . We call each 0 or 1 a bit. An $m$-valued switching function $f$ of $n$ variables $X_{1}, X_{2}, \ldots, X_{n}$ can be represented by binary vectors. If the variable $X_{i}$ is $m$-valued, the literal $X_{i}^{S_{i}}$ can be represented by a binary vector of $m$ bits:

$$
c_{i}^{0} c_{i}^{1} \cdots c_{i}^{m-1}
$$

where $c_{i}^{j}=0$ if $j \notin S_{i}$ and $c_{j}^{j}=1$ if $j \in S_{i}$. For example, for a binary logic, each literal can be represented by a vector of two bits as follows:

$X_{i}^{\varnothing}$ is represented as 00,

$X_{i}{ }^{1}$ is represented as 01 ,

$X_{i}^{0}$ is represented as 10 ,

$X_{i}^{0,1}$ is represented as 11.

For a 4-valued logic, each literal is represented by a vector of 4 bits:

$X_{i}^{0,1,2,3}$ is represented as 1111 ,

$X_{i}^{0,2}$ is represented as 1010 ,

$X_{i}^{1,3}$ is represented as 0101.

A product term of $n$ literals can be represented by $n$ such vectors. A symbol "-" is used for separating each vector. For instance, $X P, 1 X_{2}^{1}, X_{3}^{1,3}$ in cubical representation is represented by three vectors:

$$
[1100-0110-0101]
$$

which is called a cube. A cube can represent:

1. a product of literals, 
2. a sum of literals,

3. an exclusive sum of literals.

There is no difference in the representation of these forms as a cube. For example, both $X X^{0,1}+X \frac{1}{2}, 2+X \frac{1}{3}, 3$ and $X \rho, 1 \oplus X_{2}^{1,2} \oplus X_{\frac{1}{3}, 3}$ are presented as

$$
\text { [1100-0110-0101]. }
$$

Where using a cube, we should specify which form the cube used to represent. We can represent a POS, a SOP or a ESOP by an array of cubes. This way of representation is called positional notation of cube calculus (cube notation for short). In this thesis, without other specification, we use a cube to represent a product term, and use an array of cubes to represent an ESOP.

Example II.5: For binary logic, an ESOP

$$
\bar{x} \bar{y} \bar{z} \oplus \bar{x} z v \oplus y z \oplus x \bar{y} z \bar{v}
$$

in cube notation can be represented as following 4 cubes:

$$
\begin{aligned}
& {[10-10-10-11]} \\
& {[10-11-01-01]} \\
& {[11-01-01-11]} \\
& {[01-10-01-10] .}
\end{aligned}
$$

For binary logic, we can simplify the cube notation as follows:

- $\quad 00$ is represented as $\varepsilon$,

- 10 is represented as 0 ,

- 01 is represented as 1 ,

- 11 is represented as $x$.

So, the above example can also be represented as

$000 x$ 
$x 11 x$

1010.

Example II.6: For 4-valued logic, an ESOP

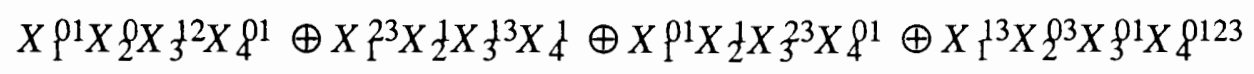

can be represented by an array of 4 cubes:

$$
\begin{aligned}
& {[1100-1000-0110-1100]} \\
& {[0011-0100-0101-0100]} \\
& {[1100-0100-0011-1100]} \\
& {[0101-1001-1100-1111] .}
\end{aligned}
$$

The $\mathrm{ON}$-array of a function can be represented by the $\mathrm{ON}$-array of cubes, and the OFF-array of a function can be represented by the OFF-array of cubes.

Example II.7: The function $f$ of Example II. 2 can be represented by the following ONarray of cubes:

001

011

101

110

111.

We can draw cubes on maps. On the map, each circle indicates one cube, as shown in Figure 4a. We can also use maps to minimize the number of cubes. For instance, 5 cubes in Figure $4 \mathrm{a}$ is reduced to 2 cubes in Figure $4 \mathrm{~b}$. In other words, we can use an ONarray of 2 cubes to express the function $f$ of Example II.2:

$x x 1$

110.

Example II.8: The function $f$ of Example II. 3 can be represented by the following ONarray of cubes: 


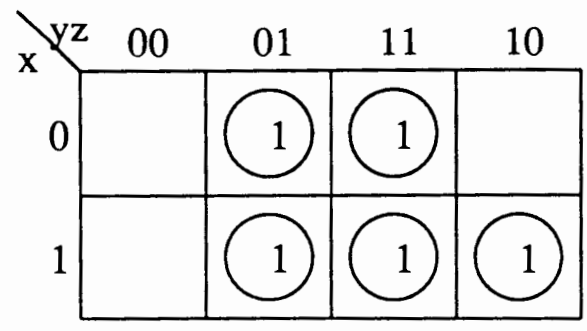

(a)

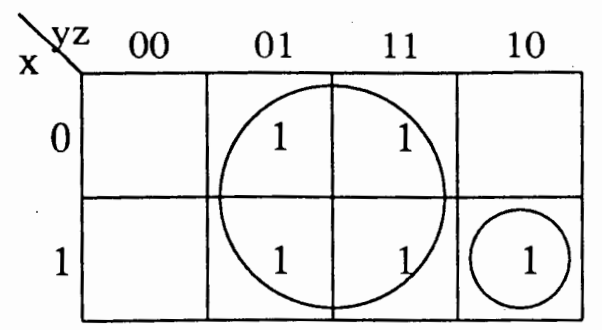

(b)

Figure 4. Karnaugh map for binary input cubes.

$$
\begin{aligned}
& {[100-010]} \\
& {[010-101]} \\
& {[001-110] .}
\end{aligned}
$$

Figure 5a shows these three cubes on the map. Please note that two circles connected by an arc denote one cube [010 - 101]. In ESOPs a true minterm can be covered by cubes an odd number of times, a false minterm can be covered an even number of times. Figure $5 b, 5 c$, and $5 d$ show other arrays of cubes representing the same function. This means that for a specific function, there are different ESOPs to represent it.

If $A$ is a cube, we write $A_{i}$ or $A$ [i] to denote the $i$-th vector of $A$. And we write $A_{i j}$ or $A[i, j]$ to denote the $j$-th bit of $A_{i}$. For instance, in Example II.8, if we denote the first cube in the array as $A$, then

$$
\begin{aligned}
& A_{1}=100, \\
& A_{2}=010, \\
& A_{11}=1, \\
& A_{12}=0, \\
& A_{13}=0, \\
& A_{21}=0, \\
& A_{22}=1,
\end{aligned}
$$




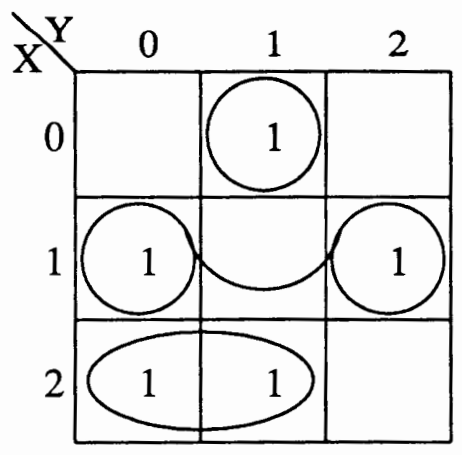

(a)

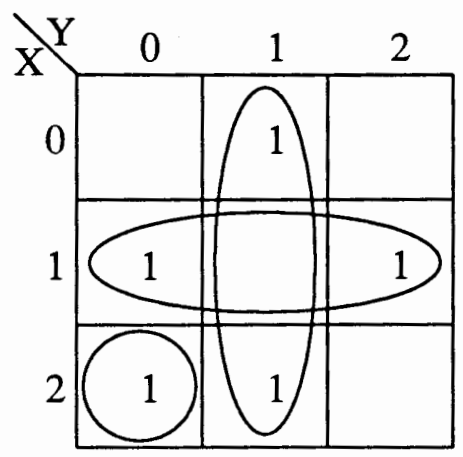

(c)

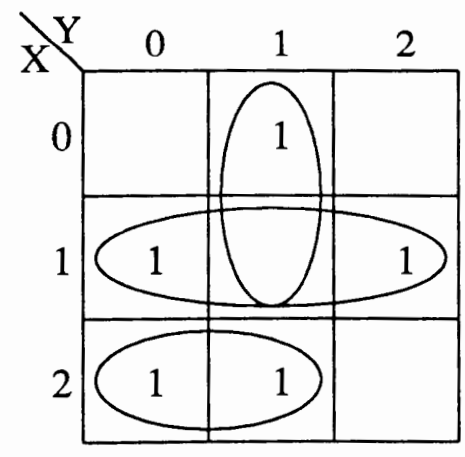

(b)

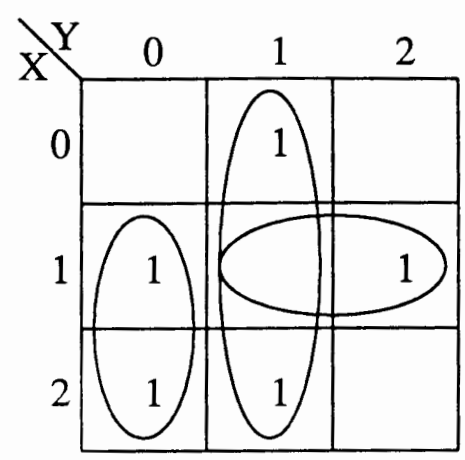

(d)

Figure 5. Map for multiple-valued input cubes.

$$
A_{23}=0 \text {. }
$$

Please note the difference between $S_{i}$ the truth set of variable $X_{i}$ and $A_{i}$ the vector in a cube corresponding to literal $X_{i}^{S_{i}}$. For a 4-valued logic, for instance, if $S_{i}=\{0,1,2\}$, the corresponding $A_{i}$ is 1110 . They represent the same literal, but in two different ways.

We define the difference of two cubes as the difference of the corresponding two terms, and the distance of two cubes as the distance of the corresponding two terms. In other words, the difference of two cubes is the number of their vectors that are different; the distance of two cubes is the number of their vectors that are disjoint. For instance, given two cubes 


$$
A=[1100-1010-0111]
$$

and

$$
B=[0110-0100-0101],
$$

the difference of two cubes is 3 , because $A_{1} \neq B_{1}, A_{2} \neq B_{2}$, and $A_{3} \neq B_{3}$. The distance of two cubes is 1 , because only $A_{2}$ and $B_{2}$ are disjoint $\left(A_{2} \cap B_{2}=\varnothing\right)$. If $A$ and $B$ are cubes, we write difference $(A, B)=r$ to indicate that their difference is $r$, and distance $(A, B)=r$ to indicate that their distance is $r$.

Given an array of cubes, if the distance of any two cubes in the array is greater than 0 , then the array of cubes is called an array of disjoint cubes. Both the arrays showed in Figures $4 \mathrm{a}$ and $4 \mathrm{~b}$ are arrays of disjoint cubes. The array showed in Figure 5a are also array of disjoint cubes. But the arrays showed in Figures $5 \mathrm{~b}, 5 \mathrm{c}$, and $5 \mathrm{~d}$ are not arrays of disjoint cubes. If an array of cubes is disjoint, its corresponding SOP expression and ESOP expression represent the same function. For instance, the disjoint cubes showed in Figure $4 \mathrm{~b}$ can be expressed as $z+x y \bar{z}$ or $z \oplus x y \bar{z}$.

\section{II.2.3. Incompletely Specified Functions}

Given a function $f: P^{n} \rightarrow Q$, if for some $p \in P^{n}$, there exists more than one $q$ in $Q$ such that $(p, q)$ is in $f$, then $f$ is an incompletely specified function.

For a binary output function, the possible output values are 0 and 1 . If the function is incompletely specified, then for some input combinations, its output value can be either 0 or 1 . In this case, the output value is a don't care value.

When we realize an incompletely specified function, we have to fix its don't care values to either 0 or 1 .

In Table III, $f$ is an incompletely specified function. $f_{1}$ and $f_{2}$ are two examples to realize this function. Function $g$ and $h$ in Table III are two extreme ways to realize the function $f$. If we fix all the don't care values in $f$ to 0 , we get $g$. On the other hand, 


\section{TABLE III}

\section{EXAMPLES OF INCOMPLETELY SPECIFIED FUNCTIONS}

\begin{tabular}{|c|c|c|c|c|c|c|}
\hline$x_{1}$ & $x_{2}$ & $g$ & $h$ & $f$ & $f_{1}$ & $f_{2}$ \\
\hline \hline 0 & 0 & 0 & 0 & 0 & 0 & 0 \\
0 & 1 & 0 & 1 & $\{0,1\}$ & 0 & 1 \\
\hline 1 & 0 & 0 & 1 & $\{0,1\}$ & 1 & 0 \\
\hline 1 & 1 & 1 & 1 & 1 & 1 & 1 \\
\hline
\end{tabular}

if we fix all the don't care values in $f$ to 1 , we get $h$. If $g$ and $h$ are so defined, then for any $p \in P_{n}$, the following relation is true:

$$
g(p) \leq f(p) \leq h(p) .
$$

This relation shows us anther way to define or explain an incompletely specified function:

Given $g: P^{n} \rightarrow Q$ and $h: P^{n} \rightarrow Q$ are two completely specified functions, such that $g(p) \leq h(p)$ for all $p \in P^{n}$. If a function $f$ is defined by the interval of $g$ and $h$,

$$
f(p)=[g(p), h(p)] \forall p \in P^{n},
$$

then $f$ is an incompletely specified function.

A multiple-valued input, binary output, incompletely specified function $f$ ( multiple-valued function, for short) is a mapping $f\left(X_{1}, X_{2}, \cdots, X_{n}\right)$ : $P_{1} \times P_{2} \times \cdots P_{n} \rightarrow Q$, where $X_{i}$ is a multiple-valued variable, $P_{i}=\left\{0,1, \ldots, p_{i}-1\right\}$ is a set of truth values that this variable may assume, and $Q=0,1, d$ ( $d$ denotes a don't care value). This is a generalization of an ordinary $\mathrm{n}$-input switching function $f: P^{n}(2) \rightarrow P(2)$.

Example II.9: Table IV is an example of a multiple-valued input binary output incompletely specified function. Figure 6 is the corresponding map.

Given an incompletely specified function, the set of those input combinations, whose associated output values are don't cares, is referred to as a $D C$-array of the func- 
TABLE IV

AN EXAMPLE OF MULTIPLE-VALUED INPUT BINARY OUTPUT INCOMPLETELY SPECIFIED FUNCTION

\begin{tabular}{cc|c}
$X$ & $Y$ & $f$ \\
\hline 0 & 0 & 0 \\
0 & 1 & 1 \\
0 & 2 & 0 \\
1 & 0 & $\{0,1\}$ \\
1 & 1 & $\{0,1\}$ \\
1 & 2 & 1 \\
2 & 0 & 1 \\
2 & 1 & 1 \\
2 & 2 & 0
\end{tabular}

\begin{tabular}{|l|l|l|l|}
$X Y$ & 0 & 1 & 2 \\
\cline { 1 - 3 } 0 & 0 & 1 & 0 \\
\hline 1 & $d$ & $d$ & 1 \\
\hline 1 & 1 & 0 \\
\hline
\end{tabular}

Figure 6. Map for a multiple-valued input incompletely specified function.

tion. The ON-array and OFF-array are defined as for completely specified functions. Figure 7 shows the ON- OFF- and DC-array of the function specified in Table IV.

\begin{tabular}{cccccc}
\multicolumn{2}{c}{ ON-array } & \multicolumn{2}{c}{ OFF-array } & \multicolumn{2}{c}{ DC-array } \\
$X$ & $Y$ & $X$ & $Y$ & $X$ & $Y$ \\
0 & 1 & 0 & 0 & 1 & 0 \\
1 & 2 & 0 & 2 & 1 & 1 \\
2 & 0 & 2 & 2 & & \\
2 & 1 & & & &
\end{tabular}

Figure 7. ON-, OFF- and DC-array of an incompletely specified function. 
The cubes in the ON-array will be called ON-cubes. Similarly, the cubes in the OFF-array and DC-array will be called $O F F$-cubes and $D C$-cubes respectively. We write $O N(f)$ to indicate an array of cubes for which $f=1, O F F(f)$ and $D C(f)$ to indicate arrays of cubes for which $f=0$ and $f=$ don't care, respectively. Figure 8 shows the different ESOPs representing the function given in Example II.9. Please note that the don't care minterms can be covered by cubes an arbitrary number of times.

Example II.10: The corresponding ESOP expressions for Figure 8 are:

Figure 8a. $\quad X^{0} Y^{1} \oplus X^{1} Y^{2} \oplus X^{2} Y^{01}$,

Figure 8b. $X^{12} Y^{01} \oplus X^{01} Y^{1} \oplus X^{1} Y^{12}$,

Figure 8c. $\quad Y^{01} \oplus X^{0} Y^{0} \oplus X^{1}$,

Figure 8d. $1 \oplus X^{01} Y^{0} \oplus X^{02} Y^{2}$.

\section{II.2.4. Multiple Output Functions}

A multiple output function is a mapping $f: P^{n} \rightarrow Q^{m}$, where $Q=\{0,1, d\}$. If $m=1$, the function is a single output function. If $m>1$, it is a multiple-output function.

One way of dealing with multiple-output functions is to represent and minimize them as a function with a single two-valued output. Let us consider a Boolean function $F$ with multiple outputs, that is, $F\left(X_{1}, \cdots, X_{n}\right)=\left(f_{0}, \ldots, f_{m-1}\right)$. We define a singleoutput switching function $F$ on $n+1$ variables where the variable $X_{n+1}$ takes the values $\{0, \ldots, m-1\} . F\left(X_{1}, \cdots, X_{n}, X_{n+1}\right)=F_{X n+1}\left(X_{1}, \cdots, X_{n}\right)$ where $F_{i}\left(X_{1}, \cdots, X_{n}\right)$ denotes the $i$-th projection of $F\left(X_{1}, \cdots, X_{n}\right)$, that is, $f_{i}$. Therefore, only single-output switching functions will be considered and $n+1$ will denote the number of input variables.

Example II.11: Given is a 3-input 2-output binary function $F(x, y, z)=\left(f_{0}, f_{1}\right)$ with binary inputs specified by Table $\mathrm{V}$. 


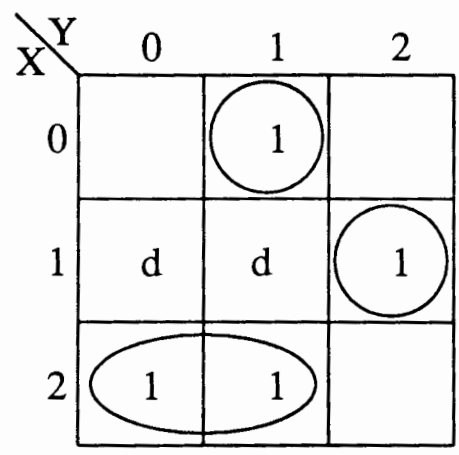

(a)

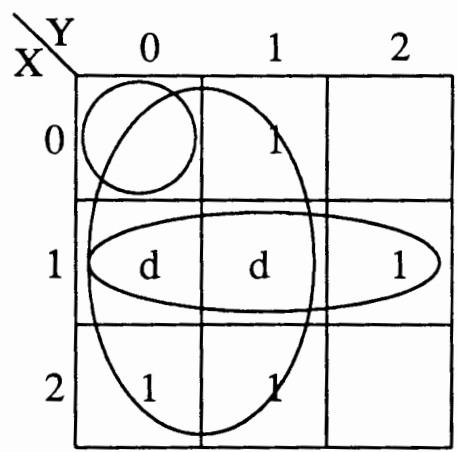

(c)

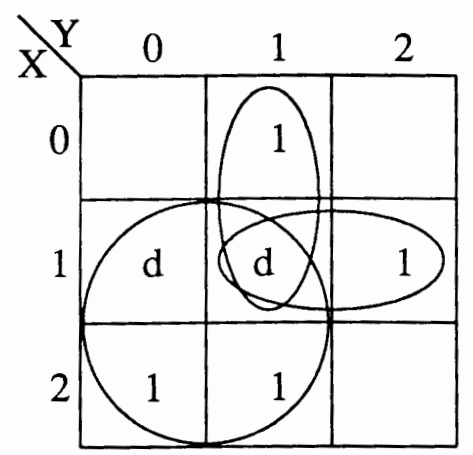

(b)

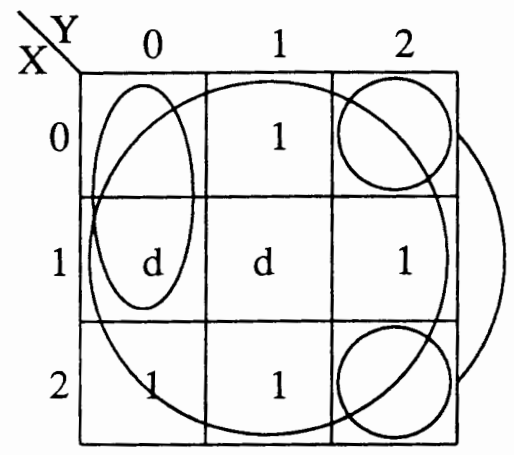

(d)

Figure 8. ESOPs for a multiple-valued input incompletely specified function.

TABLE V

AN EXAMPLE OF MULTIPLE OUTPUT FUNCTION

\begin{tabular}{ccc|cc}
$x$ & $y$ & $z$ & $f_{0}$ & $f_{1}$ \\
\hline 0 & 0 & 0 & 0 & 0 \\
0 & 0 & 1 & 1 & 0 \\
0 & 1 & 0 & 1 & 1 \\
0 & 1 & 1 & 0 & 0 \\
1 & 0 & 0 & 0 & 0 \\
1 & 0 & 1 & 1 & 0 \\
1 & 1 & 0 & 0 & 0 \\
1 & 1 & 1 & 1 & 1
\end{tabular}


This function is described by an ON-array in Table VI.

TABLE VI

ON-ARRAY OF THE MULTIPLE OUTPUT FUNCTION

\begin{tabular}{ccc|cc}
$x$ & $y$ & $z$ & $f_{0}$ & $f_{1}$ \\
\hline 0 & 0 & 1 & 1 & 0 \\
0 & 1 & 0 & 1 & 1 \\
1 & 0 & 1 & 1 & 0 \\
1 & 1 & 1 & 1 & 1
\end{tabular}

After transforming this $\mathrm{ON}$-array of the function to the $\mathrm{ON}$-array of 4-input function $F(x, y, z, v)$ with 2-valued input $v$, the $\mathrm{ON}$-array of $F$ is shown in Table VII.

TABLE VII

CONVERTING A MULTIPLE OUTPUT FUNCTION

TO A SINGLE OUTPUT FUNCTION

\begin{tabular}{llll}
$x$ & $y$ & $z$ & $v$ \\
\hline 0 & 0 & 1 & 0 \\
0 & 1 & 0 & 0 \\
0 & 1 & 0 & 1 \\
1 & 0 & 1 & 0 \\
1 & 1 & 1 & 0 \\
1 & 1 & 1 & 1
\end{tabular}

Figure $9 \mathrm{a}$ and $9 \mathrm{~b}$ are maps corresponding to Table VI. Figure $9 \mathrm{c}$ is a map corresponding to Table VII.

In Figure 10a, the ON-array of the function $F$ is covered by an array of three cubes. The corresponding ESOP expression of the function $F$ is

$$
\bar{x} y \oplus y z v \oplus z \bar{v} .
$$

We call this ESOP expression a solution of $F$. Remember that although we transform the multiple output function to a binary output function for easier handling, we have to convert it back to a multiple output function as the final result. Substituting $v=0$ to the 


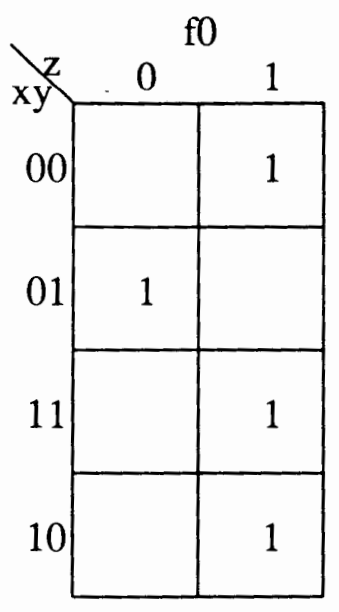

(a)

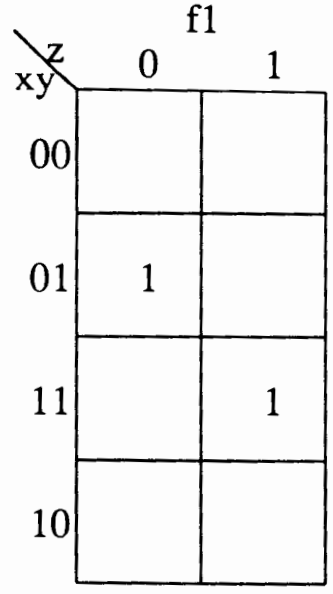

(b)

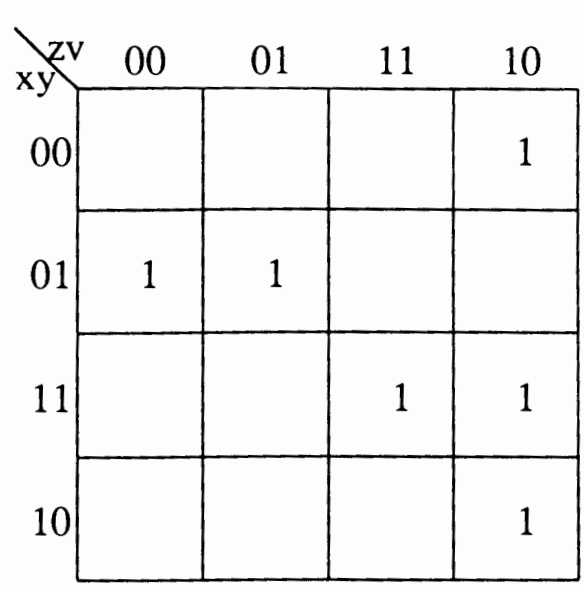

(c)

Figure 9. Map for a multiple output function.

solution of $F$, we obtain function $f_{0}(x, y, z)=\bar{x} y \oplus z$. Substituting $v=1$ we obtain $f_{1}(x, y, z)=\bar{x} y \oplus y z$. The corresponding circuit is presented in Figure 10b.

\section{II.3. OPERATIONS}

An operation is a mapping $f: P^{n} \rightarrow P$. Note that an operation is a special case of a function. For $n=1,2$, the operations are called unary, and binary respectively. For example, $f: P \rightarrow P$ is a unary operation, and $f: P^{2} \rightarrow P$ is a binary operation.

\section{II.3.1. Set Theoretic Operations}

If $P$ and $Q$ are sets, we define the union of $P$ and $Q$ as

$$
\{x \mid x \in P \text { or } x \in Q \text { or both }\} \text {. }
$$

The union of $P$ and $Q$ is denoted by $P \cup Q$. For example: $\{0,1\} \cup\{1,2\}=\{0,1,2\}$; $\{0,1\} \cup\{2,3\}=\{0,1,2,3\}$.

If $P$ and $Q$ are sets, the intersection of $P$ and $Q$, denoted by $P \cap Q$, is defined as

$$
\{x \mid x \in P \text { and } x \in Q\} \text {. }
$$




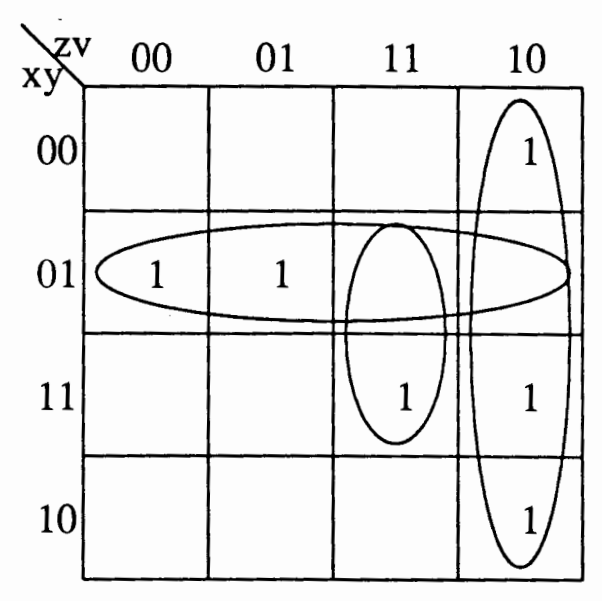

(a)

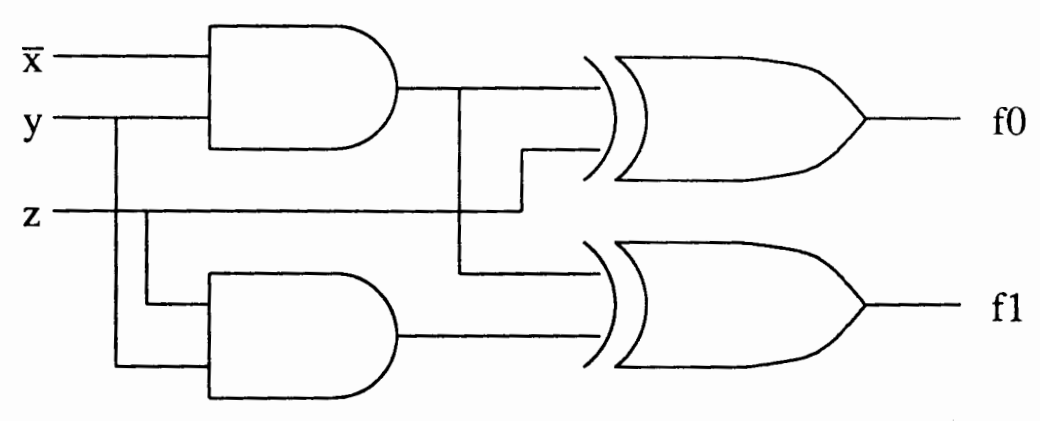

(b)

Figure 10. Map and circuit for a multiple output function.

The difference of sets $P$ and $Q$, denoted by $P-Q$ is defined as

$$
\{x \mid x \in P \text { but } x \notin Q\} \text {. }
$$

For example: $\{0,1,2\}-\{1\}=\{0,2\} ;\{0,1\}-\{2\}=\{0,1\}$.

Please do not confuse the difference of two sets and the difference of two cubes. The difference of two sets is a set operation. The result of the operation is a set which contains the elements in set $A$ but not in set $B$. The difference of two cubes (see page 18) is a function which indicates how many vectors in the cubes are different. 
If $P \cap Q=\varnothing$, we say that $P$ and $Q$ are disjoint, otherwise, they are non-disjoint. For example: sets $\{a, b\}$ and $\{c, d\}$ are disjoint; sets $\{a, b\}$ and $\{b, c\}$ are non-disjoint. If $P \not \subset Q, Q \not \subset P$, and $P \cap Q \neq \varnothing$, we say that $P$ and $Q$ are overlapping. Note that overlapping is a special case of non-disjoint. For instance, sets $\{p, q\}$ and $\{q$, $r\}$ are overlapping, sets $\{p, q, r\}$ and $\{q, r\}$ are non-disjoint, but are not overlapping. We write

$$
P \nmid Q
$$

to indicate the sets $P$ and $Q$ are overlapping.

The exclusive -or (exor for short) of sets $P$ and $Q$, denoted as $P \oplus Q$ is defined as $\{x \mid$ either $x \in P$ or $x \in Q$ but not both $\}$

By definition, $P \oplus Q=(P-Q) \cup(Q-P)$. For example: $\{1,2\} \oplus\{2,3\}=\{1,3\}$. In the case of sets $P$ and $Q$ being disjoint, $P \oplus Q=P \cup Q$.

\section{II.3.2. Cube Operations}

If $A$ is a cube, then the mapping $A^{n} \rightarrow A$ is a cube operation. We will only discuss unary and binary cube operations in this thesis.

The supercube operation of cubes $A$ and $B$ is defined as follows:

$$
A \cup B=\left[A_{1} \cup B_{1}, \ldots, A_{n} \cup B_{n}\right]
$$

where $A_{i} \cup B_{i}$ is a set union.

The intersection operation of cubes $A$ and $B$ is defined as follows:

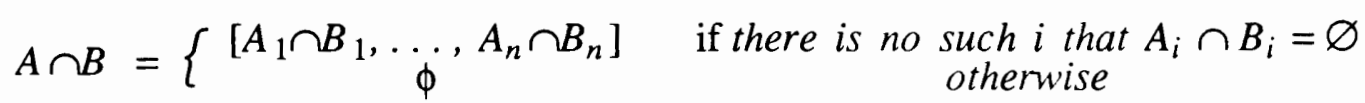

where $A_{i} \cap B_{i}$ is a set intersection, and $\phi$ is an empty cube.

Since we use a cube to represent a term, the operation of terms can be represented as the operation of cubes. For instance, if two cubes $A=\left[A_{1}, \ldots, A_{n}\right]$ and $B=$ $\left[B_{1}, \ldots, B_{n}\right]$ represent two terms $T_{S}=X_{1}^{S_{1}}, \ldots, X_{n}^{S_{n}}$ and $T_{R}=X_{1}^{R_{1}}, \ldots, X_{n}^{R_{n}}$ respectively, 
then the supercube operation of two cubes $A$ and $B$

$$
A \cup B=\left[A_{1} \cup B_{1}, \ldots, A_{i-1} \cup B_{i-1}, A_{i} \cup B_{i}, A_{i+1} \cup B_{i+1}, \ldots, A_{n} \cup B_{n}\right]
$$

is equivalent to the supercube operation of two terms $T_{S}$ and $T_{R}$

$$
T_{S} \cup T_{R}=X_{1} S_{i} \cup R_{1} \cdots X_{i-1} S_{i-1} \cup R_{i-1} X_{i} S_{i} \cup R_{i} X_{i+1} S_{i+1} \cup R_{i+1} \cdots X_{n} S_{n} \cup R_{n} .
$$

In cube notation, an operation between two variables is a local operation, and an operation between two cubes is a global operation. A local operation is a set operation, and a global operation is a cube operation. For example, the supercube operation $A \cup B$ is a global operation, and $A_{i} \cup B_{i}$ is a local operation.

Sometimes, different local or global operations may be performed on two cubes depending on a relation between them. In cube notation, a relation between two vectors (corresponding to a variable) is a local relation and a relation between two cubes (corresponding to two terms) is a global relation For instance, given two cubes $A=$ $\left[A_{1}, A_{2}, \cdots A_{n}\right]$ and $B=\left[B_{1}, B_{2}, \cdots B_{n}\right]$, then $A \subseteq B$ is a global relation, and $A_{i} \subseteq B_{i}$ is a local relation. A global relation is satisfied if all the local relations are satisfied. For example, given:

$$
A=[0011-1100-1110]
$$

and

$$
B=[1011-0110-1111]
$$

we can see that $A_{1} \subseteq B_{1}$ and $A_{3} \subseteq B_{3}$, but $A_{2} \not \subset B_{2}$. So, $A \not \subset B$.

The disjoint sharp operation on cubes $A$ and $B$ is defined as follows:

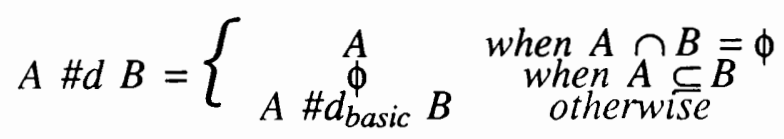

where $\mathrm{A} \# d_{\text {basic }} \mathrm{B}$ is defined as follows:

$$
\begin{array}{r}
A \# d_{\text {basic }} B=\left\{A_{1}, \cdots A_{i-1},-B_{i} \cap A_{i}, A_{i+1} \cap B_{i+1}, \cdots A_{n} \cap B_{n} \mid\right. \\
\text { for such } \left.i=1, \ldots, n, \text { that } A_{i} \not \subset B_{i}\right\}
\end{array}
$$


In this formula, $A \cap B$ and $A \subseteq B$ are global relations. If the relation $A \cap B=\phi$ is true (satisfied), the resultant cube is $A$. If the relation $A \subseteq B$ is true, an empty cube is generated. Otherwise the global operation $A \#_{d} B$ is performed. $A_{i} \not \subset B_{i}$ is a local relation, and both $A_{i} \cap B_{i}$ and $\neg B_{i} \cap A_{i}$ are local operations.

Example II.12: Given two terms $T_{1}=y v$ and $T_{2}=x z v$. They can be expressed as two cubes:

$$
\begin{aligned}
& A=[11-01-11-01] \\
& B=[01-11-01-01] .
\end{aligned}
$$

These two cubes are shown in Figure 11a. Figure 11b shows that $A \cap B \neq \phi$ (none of the vectors in resultant cube are empty sets). Next we check if the relation $A \subseteq B$ is true. Please note that $A \subseteq B$ is equivalent to $\neg A \cup B \neq \varnothing$. We can also check if the relation $A \subseteq B$ is false. This is equivalent to check if $\exists i$, such that $\neg B_{i} \cap A_{i} \neq \varnothing$. Figure $11 \mathrm{c}$ converts $B$ to $\neg B$. Here $\neg B=\left[\neg B_{1}, \cdots, \neg B_{n}\right]$ is a global operation. Figure $11 d$ intersects $A$ and $\neg B$ and shows that two of the local relations are true $\left(\neg B_{1} \cap A_{1} \neq \varnothing\right.$ and $\left.\neg B_{3} \cap A_{3} \neq \varnothing\right)$. These two local relations are indicated by two arrows. Since the relation $A \subseteq B$ is false, the operation $A \# d_{\text {basic }} B$ should be performed. Two resultant cubes are generated in Figure 11e and Figure 11f, respectively. Figure 11g shows the final results in the Karnaugh map. Note that the disjoint sharp operation of cubes $A$ and $B$ generates an array of disjoint cubes, which cover the minterms in cube $A$ but not in cube $B$. 


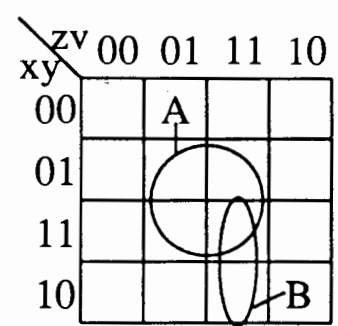

(a)

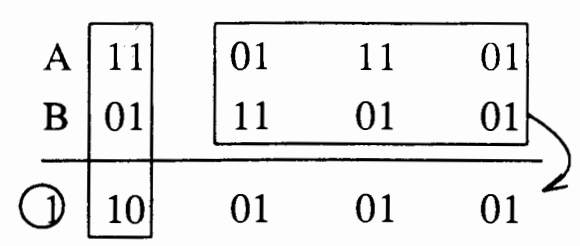

(e)

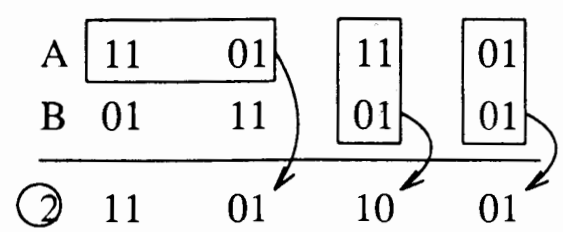

(f)

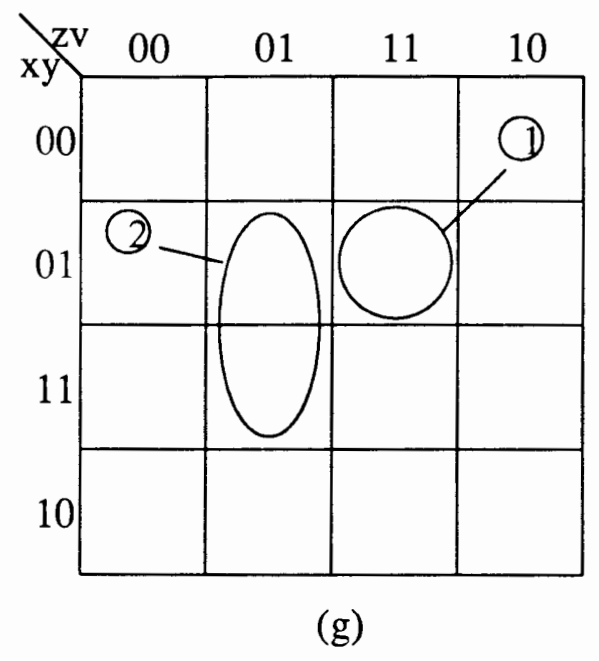

\begin{tabular}{ccccc}
$\mathrm{B}$ & 01 & 11 & 01 & 01 \\
\hline$\neg \mathrm{B}$ & 10 & 00 & 10 & 10
\end{tabular}

(c)

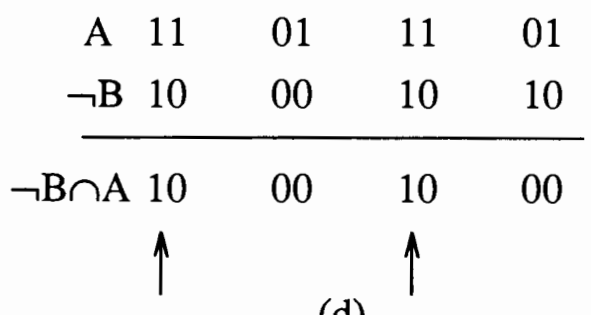

(d) (b)

\begin{tabular}{ccccc} 
A & 11 & 01 & 11 & 01 \\
B & 01 & 11 & 01 & 01 \\
\hline$A \cap B$ & 01 & 01 & 01 & 01
\end{tabular}

Figure 11. Example of a disjoint sharp operation. 


\section{CHAPTER III}

\section{MULTIPLE-VALUED INPUT EXCLUSIVE SUMS OF PRODUCTS MINIMIZATION}

\section{III.1. THE COST FUNCTIONS}

The object of logic minimization is to reduce the cost function. Our primary goal of MIESOP synthesis is to minimize the number of terms. For the circuit with the minimum number of terms our secondary goal is to minimize the total number of connections (wires). To combine these two goals together, the cost function $C$ to be used in our program is:

$$
C=N T+\frac{N I}{N I_{\text {in }}}
$$

where:

- NT is the total number of terms in the solution,

- NI is the total number of input wires to AND and EXOR gates in the solution,

- $N I_{\text {in }}$ is the total number of input wires to AND and EXOR gates in the initial function. After the function is minimized, the number of connections in the solution will be less or equal to the number of connections in the initial function. So,

$$
0<\frac{N I}{N I_{\text {in }}} \leq 1
$$

Since one term reduced in the solution will reduce the cost function by 1 , our program will selecte the solution which has minimum number of terms. Among the solutions which have the same number of terms, the program will select the one which has minimum number of connections. For instance, literal $X^{012}$ as an input to an AND gate requires a single wire for the 2-by-4 decoder realization of logic with 4-valued inputs. 
$X^{01}$ is realized as $X^{012} X^{013}$. It, therefore, requires two wires. Similarly $X^{0}=$ $X^{012} X^{013} X^{023}$ requires three wires. A product term $X^{0} Y^{1}$ requires six wires. An ESOP $X^{0} Y^{1} \oplus X^{1} Y^{0}$ contains two terms and requires 12 wires to AND gates and 2 wires to an EXOR gate. If this ESOP is our initial function, then the cost function is:

$$
C=N T+N I / N I_{\text {in }}=2+14 / 14=3 .
$$

If the ESOP is minimized as $X^{01} Y^{1} \oplus X^{1} Y^{01}$, then only 10 wires to AND gates are required. The corresponding cost function is:

$$
C=2+12 / 14=2.86 \text {. }
$$

For different technologies, different cost functions may be used. For instance, if we only concern the number of terms in the final results, then we can use the cost function $C=N T$. On the other hand, if we only concern the number of wires, then we can use the cost function $C=N I$. In chapter VI, we will try to use different cost functions to compare our results with the results from other authors.

\section{III.2. THE PROPERTIES OF THE ESOP}

Let A, B, C denote any multiple-valued input literals, or any functions on them. The following operations hold for multiple-valued input algebra:

1. Associative laws: $A \oplus(B \oplus C)=(A \oplus B) \oplus C$

2. Commutative laws: $A \oplus B=B \oplus A$

3. Identities:

3a. $A \oplus A=0$

3b. $\quad X_{i}^{S_{i}} \oplus X_{i}^{R_{i}}=X_{i}^{S_{i} \oplus R_{i}}$

3c. $X_{i} S_{i} X_{j} S_{j} \oplus X_{i}^{R_{i}} X_{j}^{R_{j}}=X_{i} S_{i} \oplus R_{i} X_{j} S_{j} \oplus X_{i}^{R_{i}} X_{j} S_{j} \oplus R_{j}$

$$
=X_{i} S_{i} \oplus R_{i} X_{j} R_{j} \oplus X_{i} S_{i} X_{j} S_{j} \oplus R_{j}
$$




\section{III.3. BASIC IDEAS TO MINIMIZE THE ESOP}

Given an ESOP, it can be represented as an array of cubes. We can do the following things to minimize the function:

\section{III.3.1. Removing Two Equal Cubes}

According to property $3 a$, if any two cubes $A$ and $B$ in the array are equal ( difference $(A, B)=0)$, they can be removed from the array.

Example III.1: The following array of four cubes

$$
\begin{aligned}
& {[0101-1111-1001-0101]} \\
& {[0101-0011-1101-1101]} \\
& {[0101-1111-1001-0101]} \\
& {[0111-1001-0001-1110]}
\end{aligned}
$$

can be reduced to an array of two cubes

$$
\begin{aligned}
& {[0101-0011-1101-1101]} \\
& {[0111-1001-0001-1110],}
\end{aligned}
$$

because the first cube and the third cube in the array are identical.

\section{III.3.2. Combining Two Cubes which Differ in One Variable}

According to property $3 b$, if any two cubes in the array differ in one variable, these two cubes can be combined to one cube.

Example III.2: Two cubes $x 101$ and $x 111$ can be combined to one cube $x 1 x 1$ as shown in Figure 12.

\section{III.3.3. Reshaping Two Cubes which Differ by 2}

If two cubes in the array differ by 2 , these two cubes can be reshaped. In other words, they can be replaced by another pair of two cubes. The number of cubes in the 


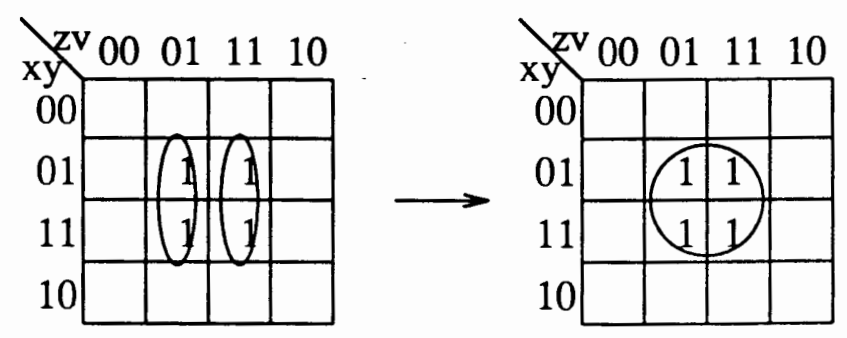

Figure 12. Combining two cubes into one cube.

array is not reduced by reshaping. However, reshaping may provide the chance to reduce the number of cubes later.

Example III.3: Given three cubes: $A=x 101, B=1111$ and $C=10 x 1$. The number of cubes can not be reduced directly, since none of them can be removed or combined. Because that the cubes $A$ and $B$ differ by two, they can be reshaped to cubes $A^{\prime}$ and $B^{\prime}$. Then cubes $B^{\prime}$ and $C$ can be combined to cube $C^{\prime}$. The number of cubes are reduced from 3 to 2. This process is shown in Figure 13.

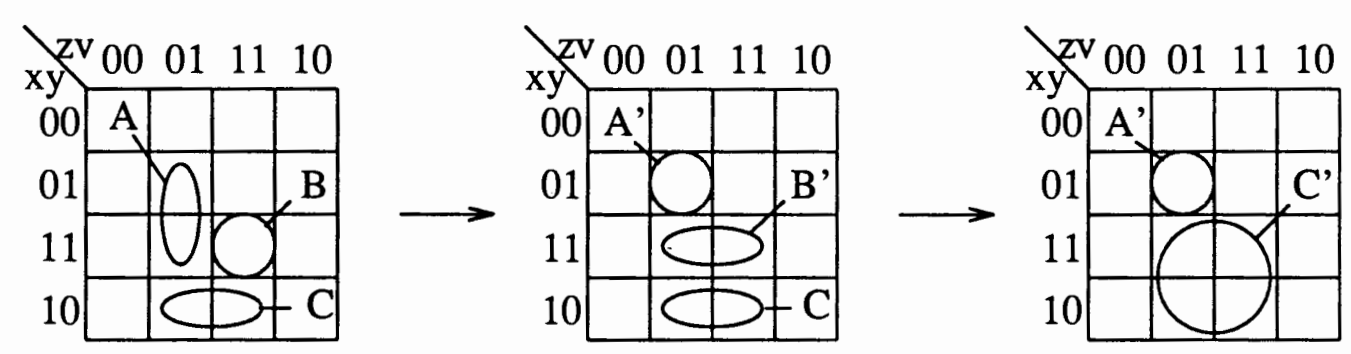

Figure 13. Reshaping two cubes.

\section{III.3.4. Increasing the Number of Cubes}

This seems contradictory to our goal: minimizing the number of cubes. However, it has been proved [Bran 91] that a set of rules (operations) can not generate a minimum form of certain ESOPs if it satisfies the following conditions:

1. Each rule changes at most two terms at a time. 
2. Each rule does not increase the number of the terms.

In other words, if we only use the first three methods (remove, combine, and reshape), our solution may be a local minimum. We will discuss how to increase the number of cubes later.

\section{III.4. THE OPERATIONS USED IN EXORCISM}

Dr. Perkowski and his former students developed an algorithm for ESOP minimization called EXORCISM [Hell 88, Perk 89]. The following operations are used in EXORCISM.

\section{III.4.1. Primary Xlinking}

Given two terms, if their truth value sets for each variable are either equal or disjoint, then these two terms are primary xlinkable. For example, terms $X^{01} Y^{0} Z^{0} U^{2} V^{13}$ and $X^{2} Y^{2} Z^{2} U^{2} V^{13}$ are primary xlinkable, because their truth value sets for each variable are either equal (variable $U$ and $V$ ) or disjoint (variable $X, Y, Z$ ). Terms $X^{01} Y^{0} Z^{0} U^{2} V^{13}$ and $X^{12} Y^{2} Z^{2} U^{2} V^{13}$ are not primary xlinkable, because their truth value sets for variable $X$ are neither equal nor disjoint.

Primary xlinking is defined by the following formula:

$$
\begin{array}{r}
T_{S} \Phi T_{R}=\oplus\left\{X_{1}^{S_{1}} \cdots X_{i-1}^{S_{i-1}} X_{i}^{S_{i} \cup R_{i}} X_{i+1}^{R_{i+1}} \cdots X_{n}^{R_{n}} \mid\right. \\
\left.\quad \text { for such } i=1, \ldots, n, \text { that } S_{i} \cap R_{i}=\varnothing\right\}
\end{array}
$$

where $T_{S}=X_{1}^{S_{1}} \ldots X_{n}^{S_{n}}$ and $T_{R}=X_{1}^{R_{1}} \ldots X_{n}^{R_{n}} \neq T_{S}$ are two terms, and all the variables have either equal or disjont truth value sets. We give the following two examples to show the primary xlinking. In both examples, 4-valued variables are assumed.

Example III.4: Given two terms $T_{1}=X^{01} Y^{1} Z^{0} V^{1}$ and $T_{2}=X^{01} Y^{0} Z^{0} V^{1}$. These two terms are primary xlinkable, since 


$$
\begin{aligned}
& \text { - for } X:\{01\}=\{01\} \\
& \text { - for } Y:\{1\} \cap\{0\}=\varnothing \\
& \text { - for } Z:\{0\}=\{0\} \\
& \text { - for } V:\{1\}=\{1\}
\end{aligned}
$$

Since the distance between two terms is 1 , only one resultant term is generated, which is:

$$
A \Phi B=X^{01} Y^{01} Z^{0} V^{1} \text {. }
$$

Figure 14 shows the procedure.

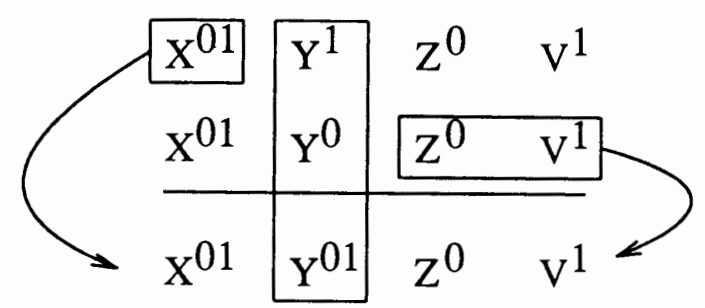

Figure 14. Procedure of distance 1 primary xlinking.

\section{Example III.5:}

Given two terms $T_{1}=X^{01} Y^{01} Z^{0} V^{1}$ and $T_{2}=X^{01} Y^{23} Z^{12} V^{1}$. These two terms are also primary xlinkable, since

$$
\begin{aligned}
& \text {-for } X:\{01\}=\{01\} \\
& \text {-for } Y:\{01\} \cap\{23\}=\varnothing \\
& \text {-for } Z:\{0\} \cap\{12\}=\varnothing \\
& \text {-for } V:\{1\}=\{1\}
\end{aligned}
$$

Since the distance between two terms is 2 , two resultant terms are generated by primary xlinking of terms $T_{1}$ and $T_{2}$. The result is:

$$
T_{1} \Phi T_{2}=X^{01} Z^{12} V^{1} \oplus X^{01} Y^{01} Z^{012} V^{1}
$$

Figure 15 shows the procedure. 


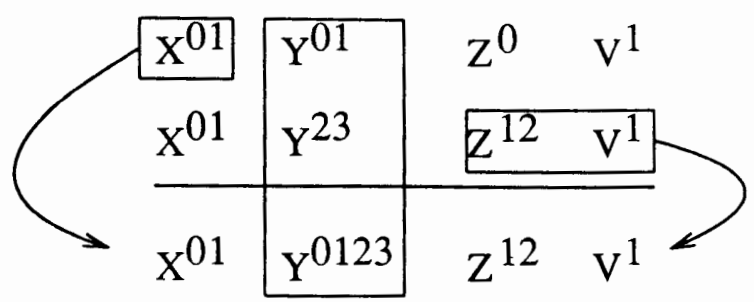

(a)

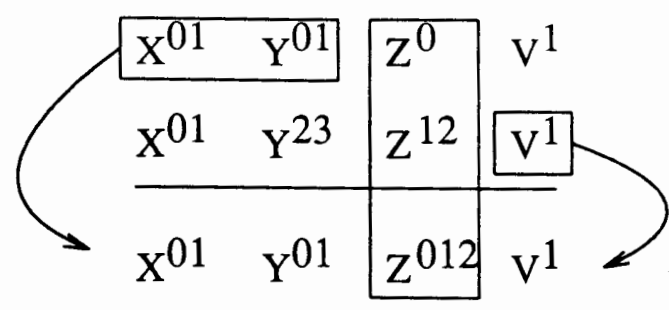

(b)

Figure 15. Procedure of distance 2 primary xlinking.

\section{III.4.2. Secondary Xlinking}

Given two terms, if there exists exactly one variable for which the truth value sets associated with one term is a sub set of the truth value set associated with the other term, and for all other variables, their truth value sets for each variable are either equal or disjoint, then these two terms are secondary xlinkable. For example, terms $X^{01} Y^{0} Z^{0} U^{2} V^{13}$ and $X^{1} Y^{2} Z^{2} U^{2} V^{13}$ are secondary xlinkable, because there is one variable $(X)$ for which one truth value set $(\{1\})$ is a sub set of another $(\{01\})$, and for all other variables, their truth value sets for each variable are either equal (variable $U$ and $V$ ) or disjoint (variable $Y, Z$ ). Terms $X^{01} Y^{0} Z^{0} U^{2} V^{13}$ and $X^{12} Y^{2} Z^{2} U^{2} V^{13}$ are not secondary xlinkable, because their truth value sets for variable $X$ are overlapping. Terms $X^{01} Y^{01} Z^{0} U^{2} V^{13}$ and $X^{1} Y^{0} Z^{2} U^{2} V^{13}$ are also not secondary xlinkable, because there are two variables $(X$ and $Y$ ) for which the truth value sets associated with one term are included in the truth value sets associated with the other term. 
Secondary xlinking is defined by the following formula:

$$
T_{S} \Theta T_{R}=T_{N} \oplus\left(T_{S} \Phi\left(T_{R} \Phi T_{N}\right)\right)
$$

where $T_{S}=X_{1}^{S_{1}} \ldots X_{n}^{S_{n}}$ and $T_{R}=X_{1}^{R_{1}} \ldots X_{n}^{R_{n}} \neq T_{S}$ are two terms; and there exists exactly one variable $X_{i}$ such that $S_{i} \supset R_{i}$ and other variables have either disjoint or equal truth value sets, and $T_{N}=X_{1}^{R_{1}} \cdots X_{i-1}^{R_{-1}} \quad X_{i}^{S_{i}-R_{i}} X_{i+1}^{R_{i+1}} \cdots X_{n}^{R_{n}}$.

We give the following two examples to show the secondary xlinking.

Example III.6: Given two terms $T_{S}=X^{01} Y^{1} Z^{0} V^{1}$ and $T_{R}=X^{1} Y^{0} Z^{0} V^{1}$. These two terms are secondary xlinkable, since

$$
\begin{aligned}
& \text { for } X:\{01\} \supset\{1\} \\
& \text { for } Y:\{1\} \cap\{0\}=\varnothing . \\
& \text { for } Z:\{0\}=\{0\} \\
& \text { for } V:\{1\}=\{1\}
\end{aligned}
$$

The distance between two cubes is 1 , but the difference between the two cubes is two, so two resultant cube are generated. The result is:

$$
T_{S} \ominus T_{R}=X^{0} Y^{0} Z^{0} V^{1} \oplus X^{01} Y^{01} Z^{0} V^{1}
$$

Figure 16 shows the procedure. In Figure $16 \mathrm{a}, T_{N}$ is generated. Figure $16 \mathrm{~b}$ shows the result of $\left(T_{R} \Phi T_{N}\right)$. Figure $16 \mathrm{c}$ shows the result of $\left(T_{S} \Phi\left(T_{R} \Phi T_{N}\right)\right)$

Example III.7: Given two terms $T_{S}=X^{01} Y^{01} Z^{0} V^{1}$ and $T_{R}=X^{1} Y^{23} Z^{12} V^{1}$. These two terms are also secondary xlinkable, since

$$
\begin{aligned}
& \text {-for } X:\{01\} \supset\{1\}, \\
& \text {-for } Y:\{01\} \cap\{23\}=\varnothing, \\
& \text {-for } Z:\{0\} \cap\{12\}=\varnothing, \\
& \text {-for } V:\{1\}=\{1\}
\end{aligned}
$$




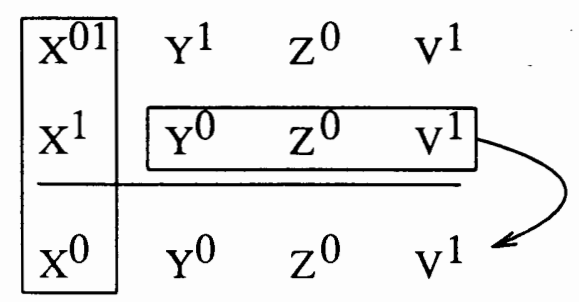

(a)

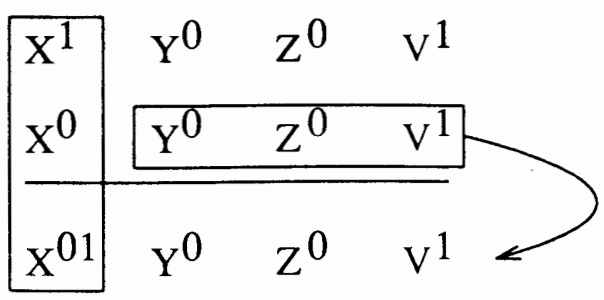

(b)

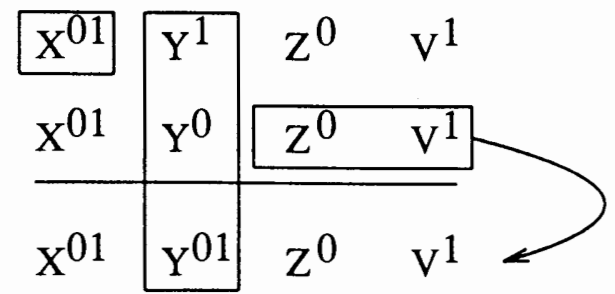

(c)

Figure 16. Procedure of distance 1 secondary xlinking.

The distance between the two terms is 2 , and the difference between the two terms is three. So, three resultant terms should be generated by secondary xlinking of terms $T_{S}$ and $T_{R}$. Figure 17 shows the procedure. In Figure 17a, $T_{N}$ is generated. In Figure 17b, $\left(T_{R} \Phi T_{N}\right)$ is generated. In figure $17 \mathrm{c}$ and figure $17 \mathrm{~d}$, two resultant cubes are generated by $\left(T_{S} \Phi\left(T_{R} \Phi T_{N}\right)\right)$. The result is:

$$
T_{S} \ominus T_{R}=X^{0} Y^{23} Z^{12} V^{1} \oplus X^{01} Z^{12} V^{1} \oplus X^{01} Y^{01} Z^{012} V^{1} .
$$

\section{III.4.3. Unlinking}

Unlinking operations are inverse to the xlinking operations. Two unlinking operations are used in EXORCISM:

1. primary unlinking,

2. secondary unlinking.

The primary unlinking is an inverse operation to the primary xlinking and the secondary unlinking is an inverse operation to the secondary xlinking. 


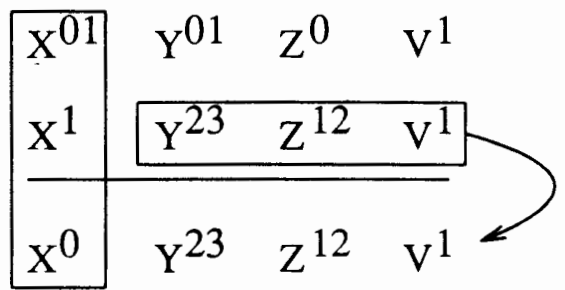

(a)

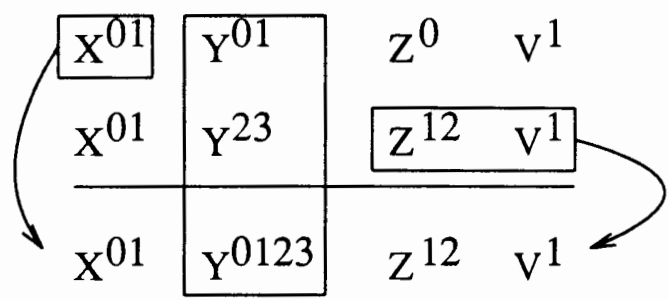

(c)

\begin{tabular}{|l|lll}
\hline$X^{1}$ & $Y^{23}$ & $Z^{12}$ & $V^{1}$ \\
$X^{0}$ & $Y^{23}$ & $Z^{12}$ & $V^{1}$ \\
\hline$X^{01}$ & $Y^{23}$ & $Z^{12}$ & $V^{1}$
\end{tabular}

(b)

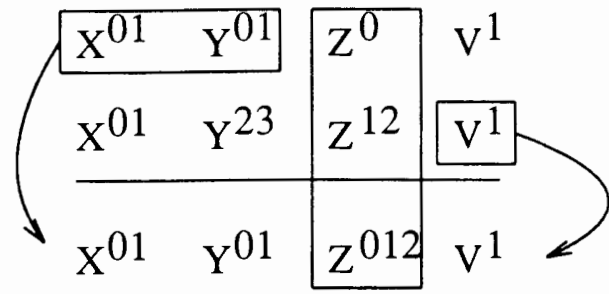

(d)

Figure 17. Procedure of distance 2 secondary xlinking.

\section{III.5. THE OPERATIONS USED IN EXMIN}

Sasao [Sasa 90a] used the following seven simplification rules in his algorithm EXMIN. The first rule, X-MERGE, can be applied if the difference of two terms is 1 . The rest of the rules can be applied if the difference of two terms is 2 and certain conditions are satisfied. In this section, we are going to show all seven rule with examples. 4valued logic is assumed for all the examples. Please note that for applying these rules, the two terms can have more than one or two literals. The number of resultant terms is equal to the difference of the two terms. Those pairs of literals which have the same truth value sets in both terms will keep the same truth value sets in the resultant terms. Those pairs of literals which have different truth value sets will generate different literals according to the corresponding rules.
(1)
X-MERGE

$$
X^{a} \oplus X^{b}=X^{(a \oplus b)}
$$


Example III.8:

$$
X^{12} Y^{01} \oplus X^{12} Y^{12}=X^{12} Y^{(01 \oplus 12)}=X^{12} Y^{02}
$$

as shown in Figure 18.

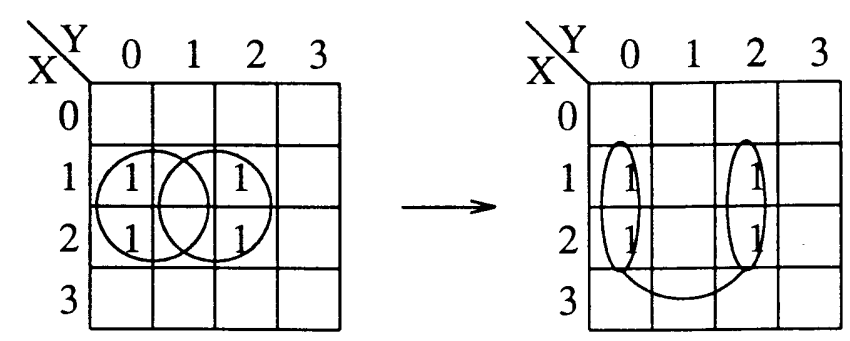

Figure 18. X-MERGE.

In example III.8, the difference of two terms is 1 . So, X-MERGE can be applied and one resultant term will be generated. The literal $X^{12}$ which is the same in both terms keeps the same truth value set in the resultant term. Another pair of literals, $Y^{01}$ and $Y^{12}$ generate the literal $Y^{02}$ in the resultant term according to the rule.

(2) RESHAPE

$$
X^{a} Y^{b} \oplus X^{c} Y^{d}=X^{a} Y^{(b \cap \bar{d})} \oplus X^{(a \cup c)} Y^{d} \quad \text { if }(a \cap c=\varnothing, b \supset d)
$$

Example III.9:

$$
X^{1} Y^{12} \oplus X^{23} Y^{1}=X^{1} Y^{2} \oplus X^{123} Y^{1}
$$

as shown in Figure 19.

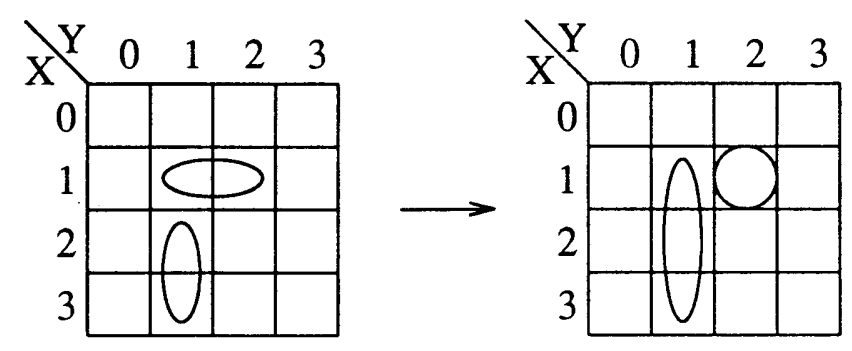

Figure 19. RESHAPE. 
(3) DUAL-COMPLEMENT

$$
X^{a} Y^{b} \oplus X^{c} Y^{d}=X^{c} Y^{(b \cap \bar{d})} \oplus X^{\overline{(a} \cap c)} Y^{b} \quad \text { if }(a \subset c, b \supset d)
$$

Example III.10:

$$
X^{1} Y^{12} \oplus X^{123} Y^{1}=X^{123} Y^{2} \oplus X^{23} Y^{12}
$$

as shown in Figure 20.

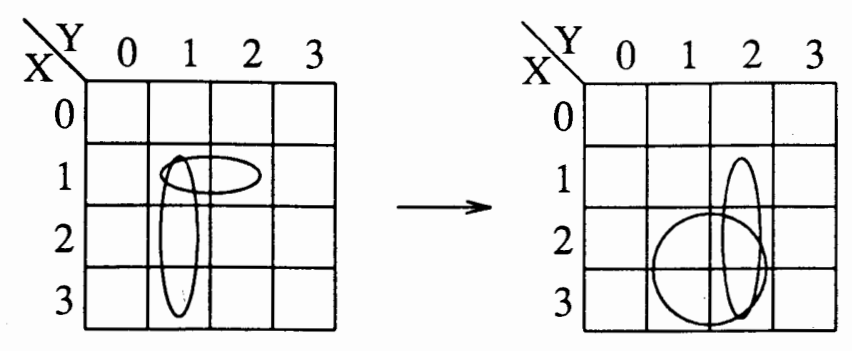

Figure 20. DUAL-COMPLEMENT.

(4) X-EXPAND-1

$$
\begin{aligned}
X^{a} Y^{b} \oplus X^{c} Y^{d}=X^{a} Y^{(b \cup d)} \oplus X^{(a \cup c)} Y^{d} & =X^{(a \cup c)} Y^{b} \oplus X^{c} Y^{(b \cup d)} \\
& \text { if }(a \cap c=\varnothing, b \cap d=\varnothing)
\end{aligned}
$$

Example III.11:

$$
X^{23} Y^{1} \oplus X^{1} Y^{2}=X^{23} Y^{12} \oplus X^{123} Y^{2}=X^{123} Y^{1} \oplus X^{1} Y^{12}
$$

as shown in Figure 21.

(5) X-EXPAND-2

$$
X^{a} Y^{b} \oplus X^{c} Y^{d}=X^{(a \cup c)} Y^{b} \oplus X^{c} Y^{(b \cap \bar{d})} \quad \text { if }(a \cap c=\varnothing, b \supset d)
$$

Example III.12:

$$
X^{1} Y^{12} \oplus X^{23} Y^{1}=X^{123} Y^{12} \oplus X^{23} Y^{2}
$$

as shown in Figure 22.

(6) X-REDUCE-1

$$
X^{a} Y^{b} \oplus X^{c} Y^{d}=X^{(a \cap \bar{c})} Y^{b} \oplus X^{c} Y^{(d \cap \bar{b})} \quad \text { if }(a \supset c, b \subset d)
$$



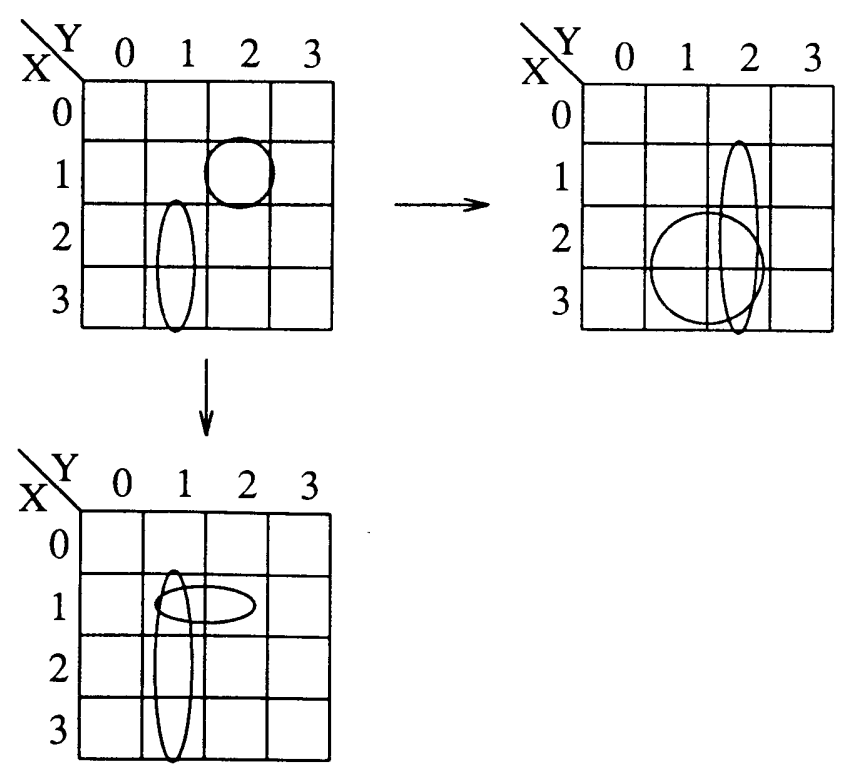

Figure 21. X-EXPAND-1.
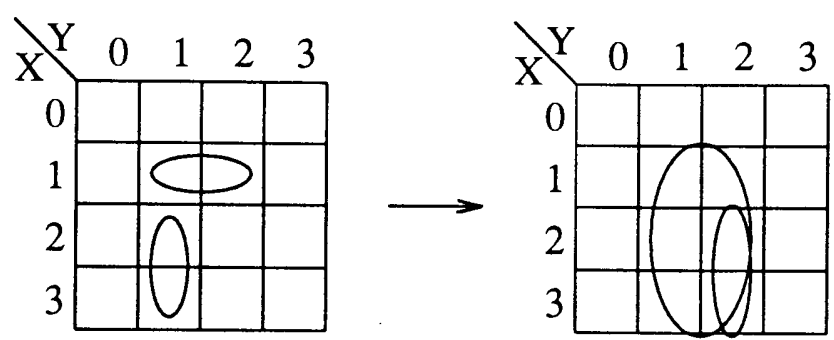

Figure 22. X-EXPAND-2.

\section{Example III.13:}

$$
X^{123} Y^{1} \oplus X^{1} Y^{12}=X^{23} Y^{1} \oplus X^{1} Y^{2}
$$

as shown in Figure 23.

(7) X-REDUCE-2

$$
\begin{array}{r}
\left.X^{a} Y^{b} \oplus X^{c} Y^{d}=X^{(a \cap \bar{c})} Y^{b} \oplus X^{c} Y^{(b \cap \bar{d})}=X^{a} Y^{(b \cap \bar{d})} \oplus X^{(a \cap \bar{c})} Y^{d}\right) \\
\text { if }(a \supset c, b \supset d)
\end{array}
$$

Example III.14:

$$
X^{123} Y^{12} \oplus X^{23} Y^{2}=X^{123} Y^{1} \oplus X^{1} Y^{2}
$$




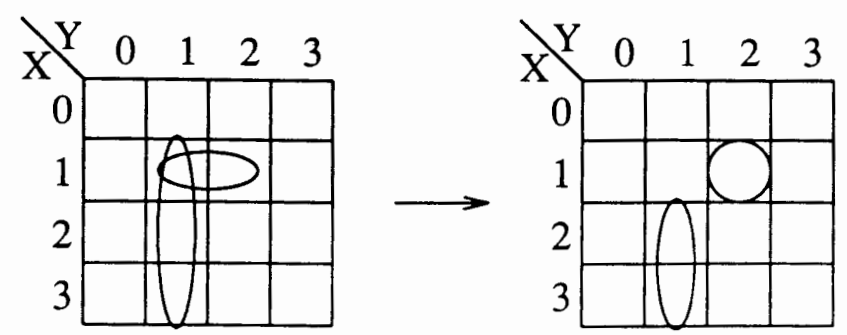

Figure 23. X-REDUCE-1.

as shown in Figure 24.
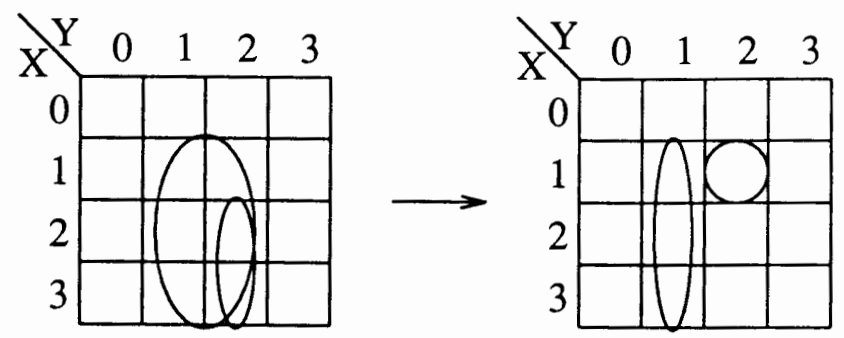

Figure 24. X-REDUCE-2. 
CHAPTER IV

\section{THE MULTIPLE-VALUED EXORLINKING OPERATION}

\section{IV.1. THE FORMULA}

Let $T_{S}=X_{1} S_{1} \ldots X_{n} S_{n}$ and $T_{R}=X_{1}{ }^{R_{1}} \ldots X_{n}{ }^{R_{n}} \neq T_{S}$ be two terms. The exorlink of terms $T_{S}$ and $T_{R}$ is defined by the following formula:

$T_{S} \otimes T_{R}=\oplus\left\{X_{1}^{S_{1}} \cdots X_{i-1}^{S_{i-1}} X_{i}^{\left(S_{i} \oplus R_{i}\right)} X_{i+1}^{R_{i+1}} \cdots X_{n}^{R_{n}} \quad\right.$ | for such $i=1, \ldots, n$, that $\left.S_{i} \neq R_{i}\right\}$

We will give a systematic procedure for finding exorlinks of full terms below. The application of this procedure will be called exorlinking. The result of the procedure will be called the exorlink of the two original full terms.

Example III.15: To find the exorlink of a pair of two full terms, $T_{S}=$ $X^{01} Y^{02} Z^{012} U^{2} V^{13}$ and $T_{R}=X^{12} Y^{12} Z^{2} U^{2} V^{13}$, in a 4-valued function, we write them vertically as shown in Figure 25a.

Each time when the polarities of the literal are different from full term to full term in the pair it is denoted by an arrow. Each arrow will give rise to one term of the exorlink. Let us now consider each arrow separately. The above initial pair of full terms can then be expanded to three resultant terms for variables $X, Y$, and $Z$ respectively, as shown in Figure 25.

1. For variable $X$, the resultant term $X^{02} Y^{12} Z^{2} U^{2} V^{13}$ is created as shown in Figure 25b. The literal $X^{02}$ in the resultant term is generated by $X^{01} \oplus X^{12}$. Other literals in the resultant term are copied from the term $T_{R}$.

2. For variable $Y$, the resultant term $X^{01} Y^{01} Z^{2} U^{2} V^{13}$ is created as shown in Figure $25 \mathrm{c}$. 


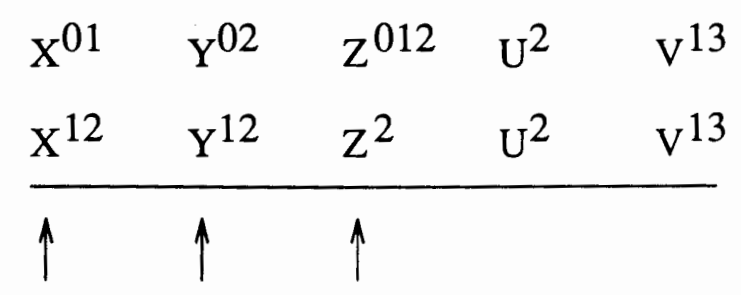

(a)

\begin{tabular}{|c|cccc}
\hline$X^{01}$ & $Y^{02}$ & $Z^{012}$ & $U^{2}$ & $V^{13}$ \\
$X^{12}$ & $Y^{12}$ & $Z^{2}$ & $U^{2}$ & $V^{13}$ \\
\hline$X^{02}$ & $Y^{12}$ & $Z^{2}$ & $U^{2}$ & $V^{13}$ \\
\hline
\end{tabular}

(b)

\begin{tabular}{|c|c|ccc|}
\hline$X^{01}$ & $Y^{02}$ & $Z^{012}$ & $U^{2}$ & $V^{13}$ \\
$X^{12}$ & $Y^{12}$ & $Z^{2}$ & $U^{2}$ & $V^{13}$ \\
\hline$X^{01}$ & $Y^{01}$ & $Z^{2}$ & $U^{2}$ & $V^{13}$
\end{tabular}

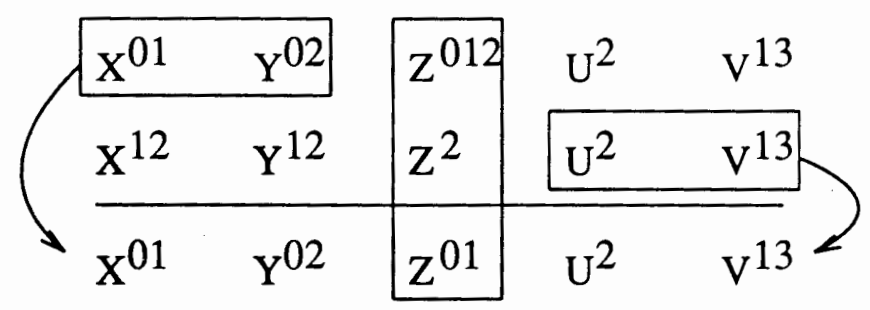

(d)

Figure 25. An example of exorlinking two terms.

3. For variable $Z$, the resultant term $X^{01} Y^{02} Z^{01} U^{2} V^{13}$ is created as shown in Figure 25d.

Under each pair of literals of different sets of values under consideration $(X$ in the first pair, $Y$ in the second, $Z$ in the third pair), we write the multiple-valued literal, with the set of truth values being the exclusive-sum of the respective sets from the literals of the terms. To create the result of exorlink for a resultant term, we copy the part of the 
term to the left of the literal from the top full term, the part to the right of the literal is copied from the bottom full term as shown. The exorlink of the initial pair of full terms is an EXOR of exorlink terms of the second order pairs for each literal of different values. Therefore

$X^{01} Y^{02} Z^{012} U^{2} V^{13} \otimes X^{12} Y^{12} Z^{2} U^{2} V^{13}=X^{02} Y^{12} Z^{2} U^{2} V^{13} \oplus X^{01} Y^{01} Z^{2} U^{2} V^{13} \oplus$ $X^{01} Y^{02} Z^{01} U^{2} V^{13}$.

This procedure can easily be further extended for any two terms in which for every two correspondingly different literals for the same variable, $X_{i} S_{i}$ and $X_{i}{ }^{R_{i}}$, the sets $S_{i}$ and $R_{i}$ are different.

Given terms $T_{S}$ and $T_{R}$, if the difference of two terms is $r$, we call $T_{S} \otimes T_{R}$ the difference r exorlinking. If the distance of two terms is $d$, we call $T_{S} \otimes T_{R}$ the distance d exorlinking.

In cube notation, a term is represented by a cube, and each literal in the term is represented by a vector. We can write the formula of exorlinking in cube notation as follows:

$A \otimes B=\oplus\left\{\left[A_{1}, \ldots, A_{i-1}, A_{i} \oplus B_{i}, B_{i+1}, \ldots, B_{n}\right] \mid\right.$ for such $i=1, \ldots, n$, that $\left.A_{i} \neq B_{i}\right\}$

The procedure of exorlinking two cubes is the same as exorlinking two terms. Figure 26 shows the exorlinking of two cubes corresponding to the two terms given in Example III.15.

Now let us prove that the exorlinking can be applied on any two cubes in the array no matter their difference.

Given two cubes $A=\left[A_{1}, A_{2}, \ldots, A_{n}\right]$ and $B=\left[B_{1}, B_{2}, \ldots, B_{n}\right]$ in the array:

1) Difference $=0$ is trival. No resultant cube will be generated according to our formula. On the other hand, if the difference of two cubes is 0 , they will be removed from the array. So, our formula is correct in this case. 


$$
\frac{1100-1010-1110-0010-0101}{0110-0110-0010-0010-0101}
$$

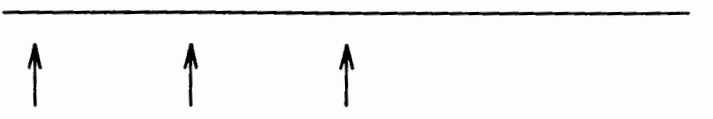

(a)

$$
\begin{aligned}
& 1100-1010-1110-0010-0101 \\
& 0110-0110-0010-0010-0101
\end{aligned}
$$

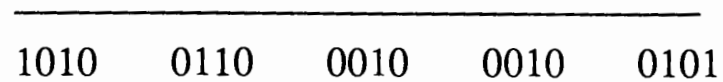

(b)

$$
\begin{aligned}
& 1100-1010-1110-0010-0101 \\
& 0110-0110-0010-0010-0101
\end{aligned}
$$

$\begin{array}{llll}1100 \quad 1100 \quad 0010 & 0010 \quad 0101\end{array}$

(c)

$$
\begin{aligned}
& 1100-1010-1110-0010-0101 \\
& 0110-0110-0010-0010-0101
\end{aligned}
$$

$\begin{array}{lllll}1100 \quad 1010 & 1100 \quad 0010 & 0101\end{array}$

(d)

Figure 26. An example of exorlinking two cubes.

2) In the case difference $=1$, without loss of generality, we can assume $A_{i} \neq B_{i}$. According to the formula,

$$
A \oplus B=\left[A_{1}, \ldots, A_{i-1}, A_{i} \oplus B_{i}, B_{i+1}, \ldots, B_{n}\right] .
$$

On the other hand,

$$
A \otimes B=A \oplus B=\left[A_{1}, A_{2}, \ldots, A_{i}, \ldots, A_{n}\right] \oplus\left[B_{1}, B_{2}, \ldots, B_{i}, \ldots, B_{n}\right] .
$$

Since $A_{j}=B_{j}$ when $i \neq j$, we can rewrite $A$ and $B$ as

$$
A=\left[A_{1}, \ldots, A_{i}, B_{i+1}, \ldots, B_{n}\right]
$$

and

$$
B=\left[A_{1}, \ldots, A_{i-1}, B_{i}, \ldots, B_{n}\right] .
$$

According to distributive law: $A B \oplus A C=A(B \oplus C)$, we have 
$A B D \oplus A C D=(A B \oplus A C) D=A(B \oplus C) D$

So,

$$
\begin{aligned}
A \oplus B & =\left[A_{1}, \ldots, A_{i}, B_{i+1}, \ldots, B_{n}\right] \oplus\left[A_{1}, \ldots, A_{i-1}, B_{i}, \ldots, B_{n}\right] \\
& =\left[A_{1}, \ldots, A_{i-1}, A_{i} \oplus B_{i} B_{i+1}, \ldots, B_{n}\right] .
\end{aligned}
$$

3) Assume our formula is correct when difference $=i, i \geq 1$. Without loss of generality, let us assume $A_{j} \neq B_{j}$ when $j \leq i$ and $A_{j}=B_{j}$ when $j>i$. Accordign to our formula, $A \otimes B$ will generate the following resultant cubes:

$$
\begin{aligned}
& {\left[A_{1} \oplus B_{1}, B_{2}, \ldots, B_{n}\right]} \\
& {\left[A_{1}, A_{2} \oplus B_{2}, B_{3}, \ldots, B_{n}\right]} \\
& \ldots \\
& {\left[A_{1}, \ldots, A_{i-1}, A_{i} \oplus B_{i}, B_{i+1}, \ldots, B_{n}\right] .}
\end{aligned}
$$

4) We will prove that when difference $=i+1$, our formula is also correct. Given two cubes $A^{\prime}$ and $B^{\prime} . A^{\prime}=\left[A_{1}, \ldots, A_{i}, \ldots, A^{\prime}{ }_{k}, \ldots, A_{n}\right]$ and $B^{\prime}=\left[B_{1}, \ldots, B_{i}, \ldots, B_{k}^{\prime}, \ldots\right.$, $B_{n}$ ]. The first $i$ literals in the two cubes are different, as in the case of cubes $A$ and $B$. Assume $A^{\prime}{ }_{k} \neq B_{k}^{\prime}$ and $i<k \leq n$. So, difference $\left(A^{\prime}, B^{\prime}\right)=i+1$. We create a cube $A^{\prime \prime}=\left[A_{1}, \ldots, A_{i}, \ldots, B_{k}^{\prime}, \ldots, A_{n}\right]$. All the literals in $A^{\prime \prime}$ are the same as in $A^{\prime}$, except that $A^{\prime}{ }_{k}$ is substituted by $B^{\prime}{ }_{k}$.

Since $A \oplus A=0$, we have

$$
A^{\prime} \oplus B^{\prime}=A^{\prime} \oplus A^{\prime \prime} \oplus A^{\prime \prime} \oplus B^{\prime} .
$$

Since difference $\left(A^{\prime \prime}, B^{\prime}\right)=i$, according to 3$), A^{\prime \prime} \oplus B^{\prime}=$

$$
\begin{aligned}
& {\left[A_{1} \oplus B_{1}, B_{2}, \ldots, B_{n}\right]} \\
& {\left[A_{1}, A_{2} \oplus B_{2}, B_{3}, \ldots, B_{n}\right]} \\
& \ldots \\
& {\left[A_{1}, \ldots, A_{i-1}, A_{i} \oplus B_{i}, B_{i+1}, \ldots, B_{n}\right] .}
\end{aligned}
$$

Since difference $\left(A^{\prime}, A^{\prime \prime}\right)=1$, according to 2$), A^{\prime} \oplus A^{\prime \prime}=$

$$
\left[A_{1}, \ldots, A_{k}^{\prime} \oplus B_{k}^{\prime}, \ldots, B_{n}\right]
$$


So, $A^{\prime \prime} \oplus B^{\prime \prime}=$

$$
\begin{aligned}
& {\left[A_{1} \oplus B_{1}, B_{2}, \ldots, B_{n}\right]} \\
& {\left[A_{1}, A_{2} \oplus B_{2}, B_{3}, \ldots, B_{n}\right]} \\
& \ldots \\
& {\left[A_{1}, \ldots, A_{i-1}, A_{i} \oplus B_{i}, B_{i+1}, \ldots, B_{n}\right]} \\
& {\left[A_{1}, \ldots, A_{k}^{\prime} \oplus B^{\prime}{ }_{k}, \ldots, B_{n}\right] .}
\end{aligned}
$$

These are exactly the same resultant cubes our formula will generate. So, our formula is correct no matter the difference of the two given cubes.

In the rest of this chapter, we will discuss difference 1, difference 2 , and difference 3 exorlinking. These operations are used in our EXORCISM-MV-2 algorithm. We will also compare exorlinking with xlinking [Hell 88, Perk 89] and the operations in EXMIN [Sasa 90a]. For convenience, we will use terms in equations, and use cube notation in maps.

\section{IV.2. DIFFERENCE 1 EXORLINKING}

Given two terms $T_{S}$ and $T_{R}$, assume $X^{S_{i}}$ and $X^{R_{i}}$ are a pair of literals in terms $T_{S}$ and $T_{R}$, respectively. Assume $X^{S_{i}} \neq X^{R_{i}}$ and other pairs of literals in the two terms are equal. Then these two terms are difference 1 exorlinkable. Difference 1 exorlinking of two terms generates one resultant term. When the difference of two terms is 1 , the distance of the two terms can be 0 or 1 .

\section{IV.2.1. Difference 1 Distance 1 Exorlinking}

Example III.16: Let $T_{S}=X^{23} Y^{23}$, and $T_{R}=X^{1} Y^{23}$.

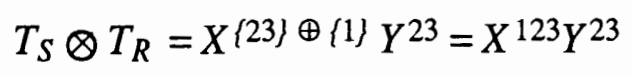

This operation is equivalent to distance 1 primary xlinking of EXORCISM [Hell 88, Perk 89], and X-MERGE of EXMIN [Sasa 90a]. 


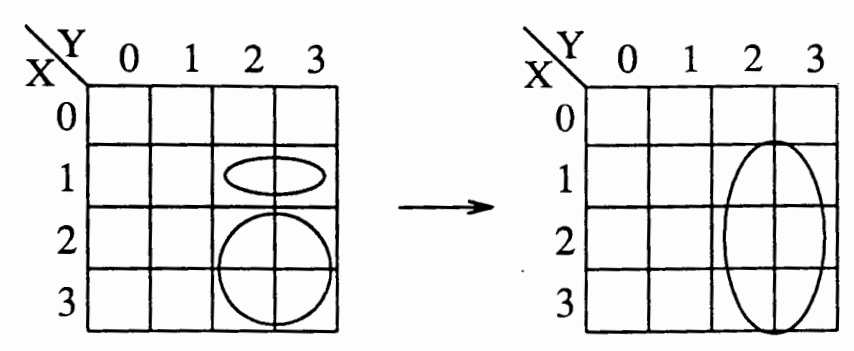

Figure 27. Difference 1 distance 1 exorlinking.

IV.2.2. Difference 1 Distance 0 Exorlinking

When the difference of two terms is 1 and the distance of two terms is 0 , there are two possible cases: the two truth sets are overlapped, or one of the truth sets is contained in the other.

Example III.17: Let $T_{S}=X^{123} Y^{23}$ and $T_{R}=X^{1} Y^{23}$.

$$
T_{S} \otimes T_{R}=X^{\{123\} \oplus\{1\}} Y^{23}=X^{23} Y^{23}
$$

The truth set of $X$ in term $T_{R}$ is contained by the truth set of $X$ in term $T_{S}$. The operation is shown in Figure 28.

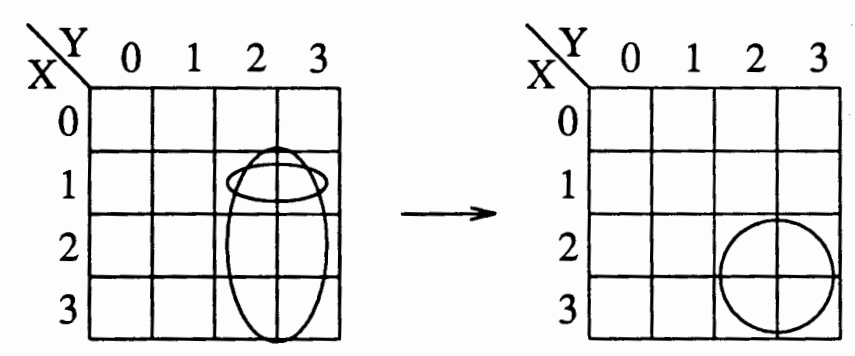

Figure 28. An example of difference 1 distance 0 exorlinking $\left(S_{i} \supset R_{i}\right)$.

Example III.18: Let $T_{S}=X^{123} Y^{23}$ and $T_{R}=X^{01} Y^{23}$.

$$
T_{S} \otimes T_{R}=X^{\{123\} \oplus\{01\} Y^{23}=X^{023} Y^{23}}
$$

The truth sets of $X$ in terms $T_{S}$ and $T_{R}$ are overlapped (denoted as $S_{i} \backslash R_{i}$ ). The opera- 
tion is shown in Figure 29.

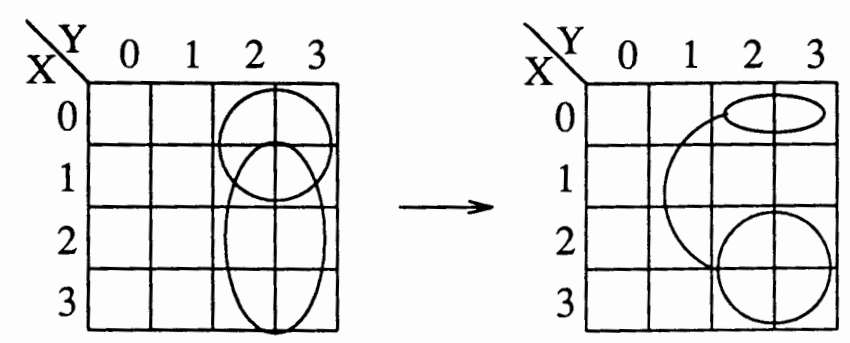

Figure 29. An example of difference 1 distance 0 exorlinking $\left(S_{i} \backslash R_{i}\right)$.

This operation is equivalent to X-MERGE of EXMIN [Sasa 90a].

\section{IV.3. DIFFERENCE 2 EXORLINKING}

Given two terms, if two pairs of their truth sets are different (assume $X^{S_{i}} \neq X^{R_{i}}$, and $Y^{S_{j}} \neq Y^{R_{j}}$ ), then difference 2 exorlink can be performed, and two resultant terms will be generated.

Let us observe that difference 2 exorlink of two terms $T_{S}$ and $T_{R}$ is different from the difference 2 exorlink of two terms $T_{R}$ and $T_{S}$.

Example III.19: Given two terms $T_{S}=X^{013} Y^{13}$ and $T_{R}=X^{23} Y^{01}$ :

$$
\begin{aligned}
& T_{S} \otimes T_{R}=X^{013} Y^{13} \otimes X^{23} Y^{01}=X^{012} Y^{01} \oplus X^{013} Y^{03} \\
& T_{R} \otimes T_{S}=X^{23} Y^{01} \otimes X^{013} Y^{13}=X^{012} Y^{13} \oplus X^{23} Y^{03}
\end{aligned}
$$

The procedures of these two operations are shown in Figure 30a and 30b.

As we discussed in section II.3.1 (see page 27), if the truth sets $S_{i}$ and $R_{i}$ are different, there are three possibilities:

$$
\begin{aligned}
& -S_{i} \cap R_{i}=\varnothing, \\
& \text { - } S_{i} \backslash R_{i}, \\
& \text { - } S_{i} \supset R_{i} \text { or } R_{i} \supset S_{i} .
\end{aligned}
$$




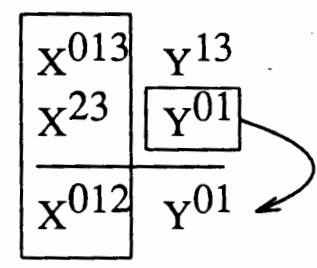

(a)
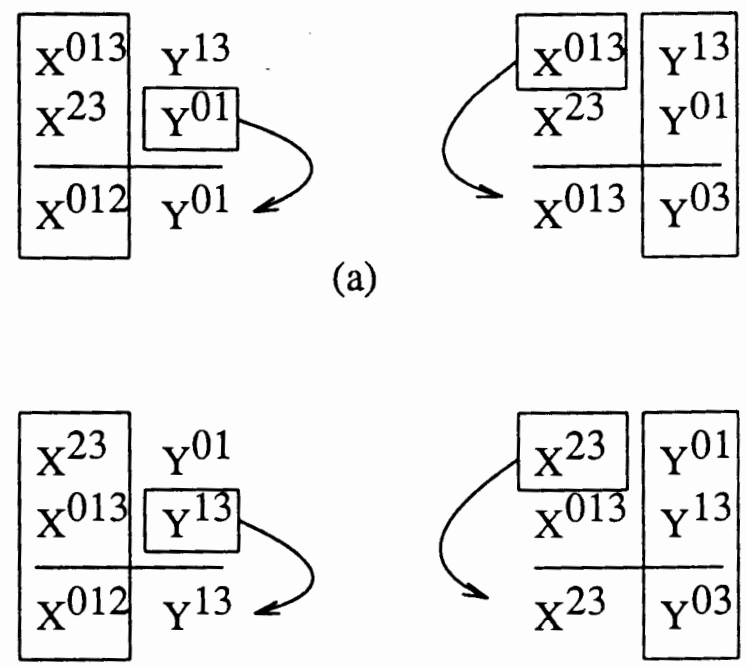

(b)

Figure 30. Difference 2 exorlinking $\left(T_{S} \otimes T_{R}\right.$ and $\left.T_{R} \otimes T_{S}\right)$.

In the case of difference 2 , there are many different combinations for relations $S_{i}, R_{i}$ and $S_{j}, R_{j}$. Next, we are going to discuss each of these cases by showing examples.

1. $S_{i} \cap R_{i}=\varnothing, S_{j} \cap R_{j}=\varnothing$

Given $T_{S}=X^{23} Y^{1}$, and $T_{R}=X^{1} Y^{2}$

$$
T_{S} \otimes T_{R}=X^{23} Y^{1} \otimes X^{1} Y^{2}=X^{123} Y^{2} \oplus X^{23} Y^{12}
$$

as shown in Figure 31a.

$$
T_{R} \otimes T_{S}=X^{1} Y^{2} \otimes X^{23} Y^{1}=X^{123} Y^{1} \oplus X^{1} Y^{12}
$$

as shown in Figure $31 \mathrm{~b}$.

This operation is equivalent to distance 2 primary xlinking of EXORCISM [Perk 89], and X-EXPAND-1 of EXMIN [Sasa 90a].

2. $S_{i} \cap R_{i}=\varnothing, S_{j} \supset R_{j}$

Given $T_{S}=X^{23} Y^{12}$, and $T_{R}=X^{1} Y^{2}$

$$
T_{S} \otimes T_{R}=X^{23} Y^{12} \otimes X^{1} Y^{2}=X^{123} Y^{2} \oplus X^{23} Y^{1}
$$

as shown in Figure 32a. 


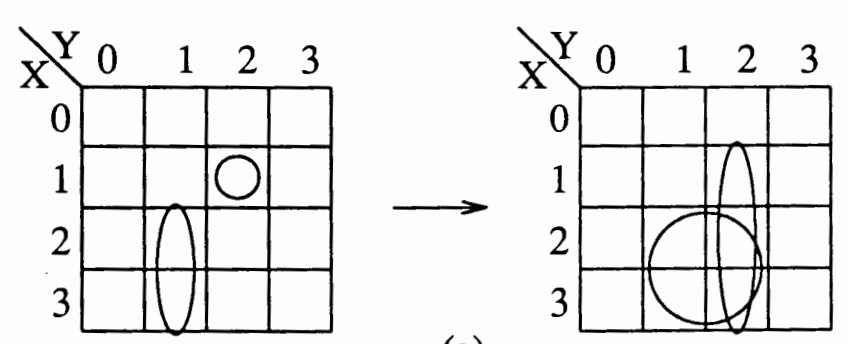

(a)
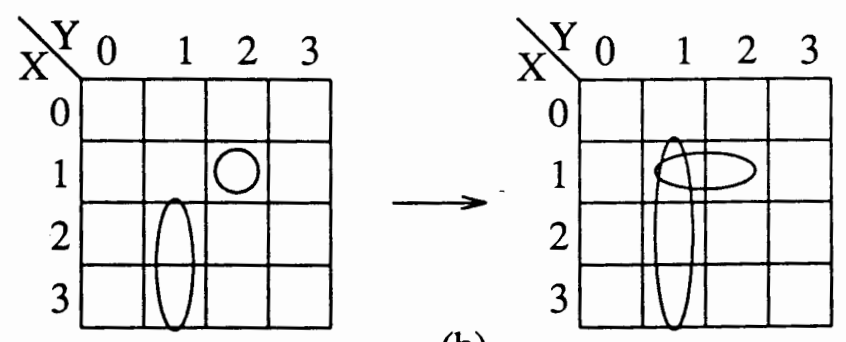

(b)

Figure 31. Difference 2 exorlinking $\left(S_{i} \cap R_{i}=\varnothing, S_{j} \cap R_{j}=\varnothing\right)$.
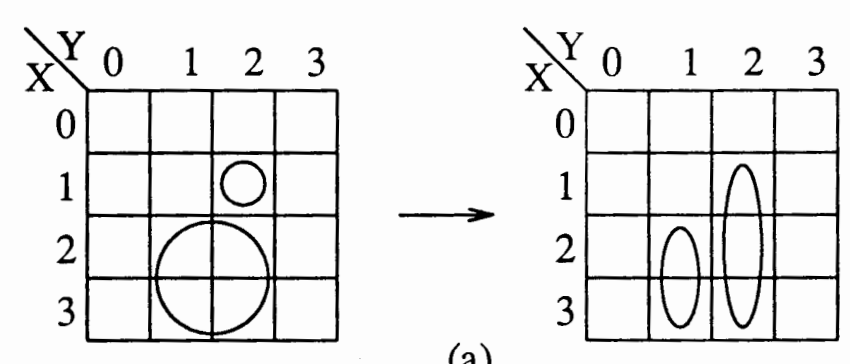

(a)
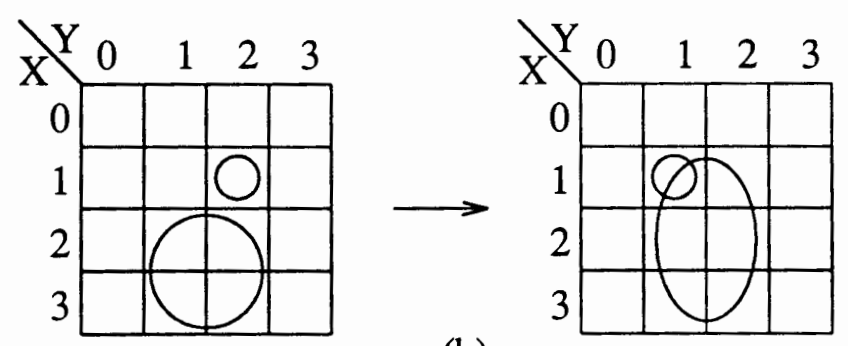

(b)

Figure 32. Difference 2 exorlinking $\left(S_{i} \cap R_{i}=\varnothing, S_{j} \supset R_{j}\right)$.

$$
T_{R} \otimes T_{S}=X^{1} Y^{2} \otimes X^{23} Y^{12}=X^{123} Y^{12} \oplus X^{1} Y^{1}
$$

as shown in Figure 32b. 
The operation RESHAPE of EXMIN [Sasa 90a] generates the same result as Figure 32a. The operation distance 1 secondary xlinking of EXORCISM [Perk 89], and XEXPAND-2 of EXMIN [Sasa 90a] generate the same result as Figure 32b.

3. $S_{i} \cap R_{i}=\varnothing, S_{j} \backslash R_{j}$

Given $T_{S}=X^{23} Y^{12}$, and $T_{R}=X^{1} Y^{23}$

$T_{S} \otimes T_{R}=X^{23} Y^{12} \otimes X^{1} Y^{23}=X^{123} Y^{23} \oplus X^{23} Y^{13}$

as shown in Figure 33a.

$T_{R} \otimes T_{S}=X^{1} Y^{23} \otimes X^{23} Y^{12}=X^{123} Y^{12} \oplus X^{1} Y^{13}$

as shown in Figure 33b.
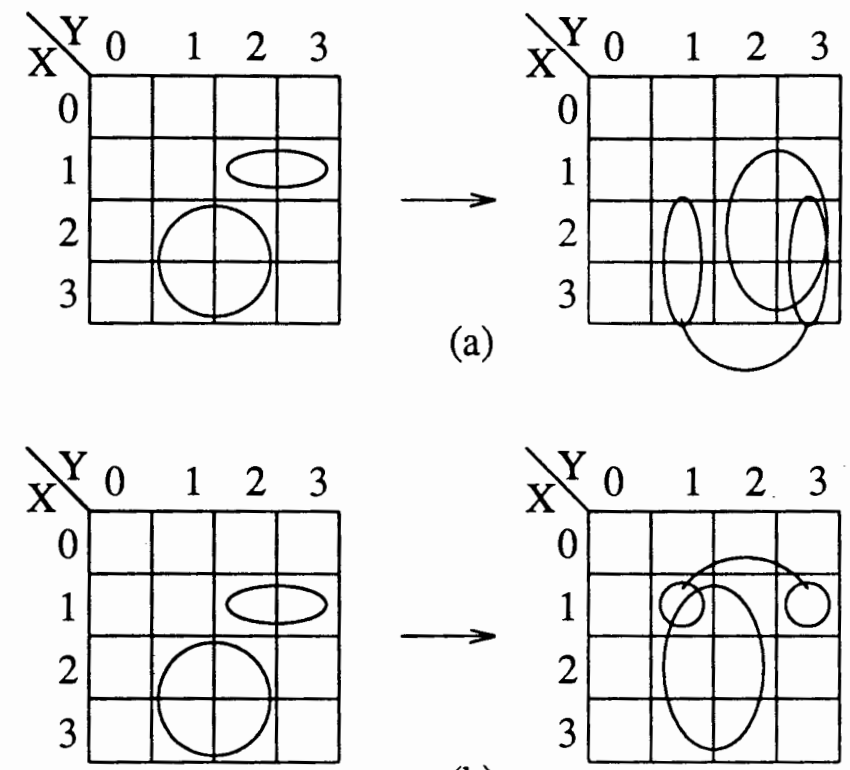

(b)

Figure 33. Difference 2 exorlinking $\left(S_{i} \cap R_{i}=\varnothing, S_{j} \backslash R_{j}\right)$.

No compatible operation can be found in the previous literatrue.

4. $S_{i} \supset R_{i}, S_{j} \supset R_{j}$

Given $T_{S}=X^{123} Y^{12}$, and $T_{R}=X^{23} Y^{2}$

$$
T_{S} \otimes T_{R}=X^{123} Y^{12} \otimes X^{23} Y^{2}=X^{1} Y^{2} \oplus X^{123} Y^{1}
$$


as shown in Figure 34a.

$$
T_{R} \otimes T_{S}=X^{23} Y^{2} \otimes X^{123} Y^{12}=X^{1} Y^{12} \oplus X^{23} Y^{1}
$$

as shown in Figure 34b.
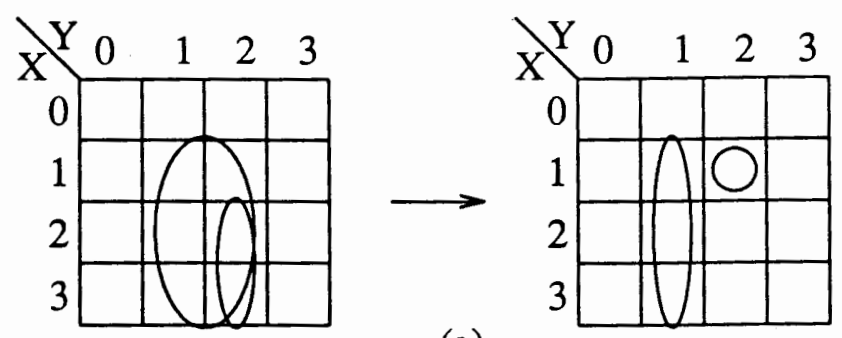

(a)
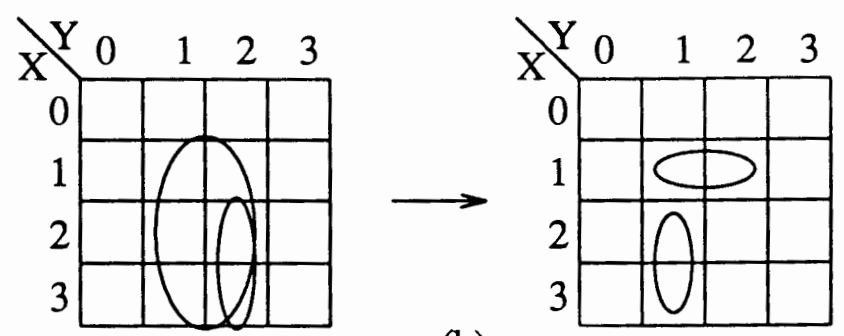

(b)

Figure 34. Difference 2 exorlinking $\left(S_{i} \supset R_{i}, S_{j} \supset R_{j}\right)$.

This operation is equivalent to the secondary unlink of EXORCISM [Perk 89] and XREDUCE-2 of EXMIN [Sasa 90a].

5. $S_{i} \supset R_{i}, S_{j} \subset R_{j}$

Given $T_{S}=X^{123} Y^{1}$, and $T_{R}=X^{1} Y^{12}$

$$
T_{S} \otimes T_{R}=X^{123} Y^{1} \otimes X^{1} Y^{12}=X^{23} Y^{12} \oplus X^{123} Y^{2}
$$

as shown in Figure 35a.

This operation is equivalent to DUAL-COMPLEMENT of EXMIN [Sasa 90a].

$$
T_{R} \otimes T_{S}=X^{1} Y^{12} \otimes X^{123} Y^{1}=X^{23} Y^{1} \oplus X^{1} Y^{2}
$$

as shown in Figure 35b.

This operation is equivalent to primary unlink of EXORCISM [Perk 89], and X- 

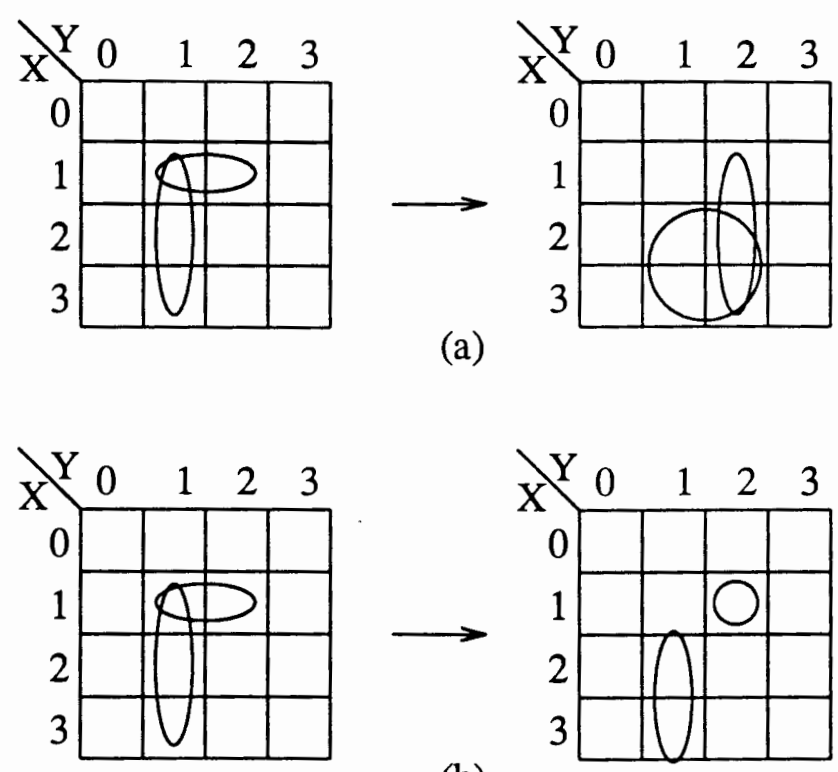

(b)

Figure 35. Difference 2 exorlinking $\left(S_{i} \supset R_{i}, S_{j} \subset R_{j}\right)$.

REDUCE-1 of EXMIN [Sasa 90a].

6. $S_{i} \supset R_{i}, S_{j} \backslash R_{j}$

Given $T_{S}=X^{123} Y^{12}$, and $T_{R}=X^{1} Y^{23}$

$$
T_{S} \otimes T_{R}=X^{123} Y^{12} \otimes X^{1} Y^{23}=X^{23} Y^{23} \oplus X^{123} Y^{13}
$$

as shown in Figure 36a.

$$
T_{R} \otimes T_{S}=X^{1} Y^{23} \otimes X^{123} Y^{12}=X^{23} Y^{12} \oplus X^{1} Y^{13}
$$

as shown in Figure 36b.

No compatible operation can be found in the previous literature.

7. $S_{i} \backslash R_{i}, S_{j} \backslash R_{j}$

Given $T_{S}=X^{23} Y^{12}$, and $T_{R}=X^{12} Y^{23}$

$$
X^{23} Y^{12} \oplus X^{12} Y^{23}=X^{13} Y^{23} \oplus X^{23} Y^{13}
$$

as shown in Figure 37a.

$$
X^{12} Y^{23} \oplus X^{23} Y^{12}=X^{13} Y^{12} \oplus X^{12} Y^{13}
$$



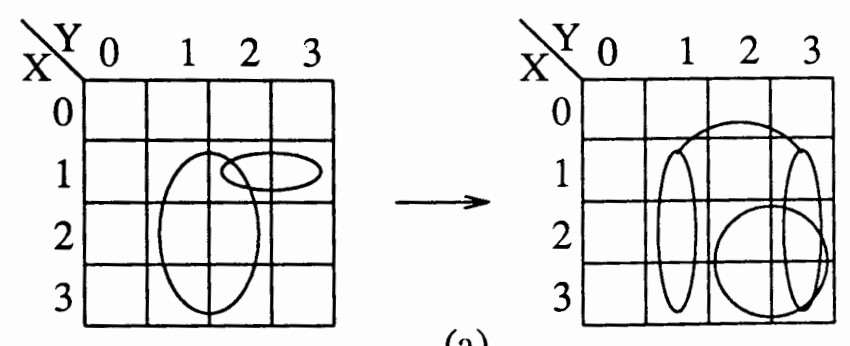

(a)
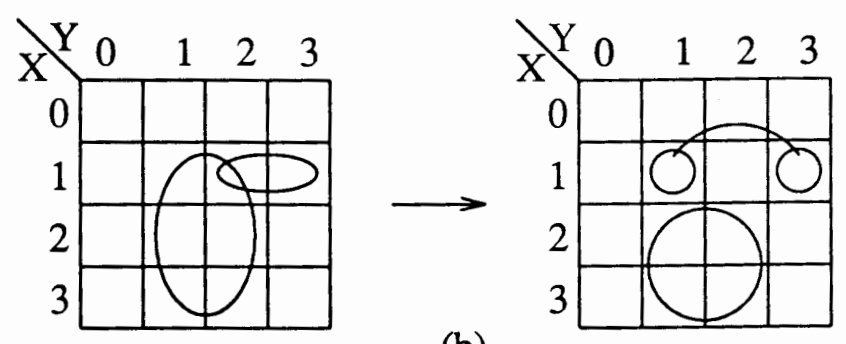

(b)

Figure 36. Difference 2 exorlinking $\left(S_{i} \supset R_{i}, S_{j} \backslash R_{j}\right)$.
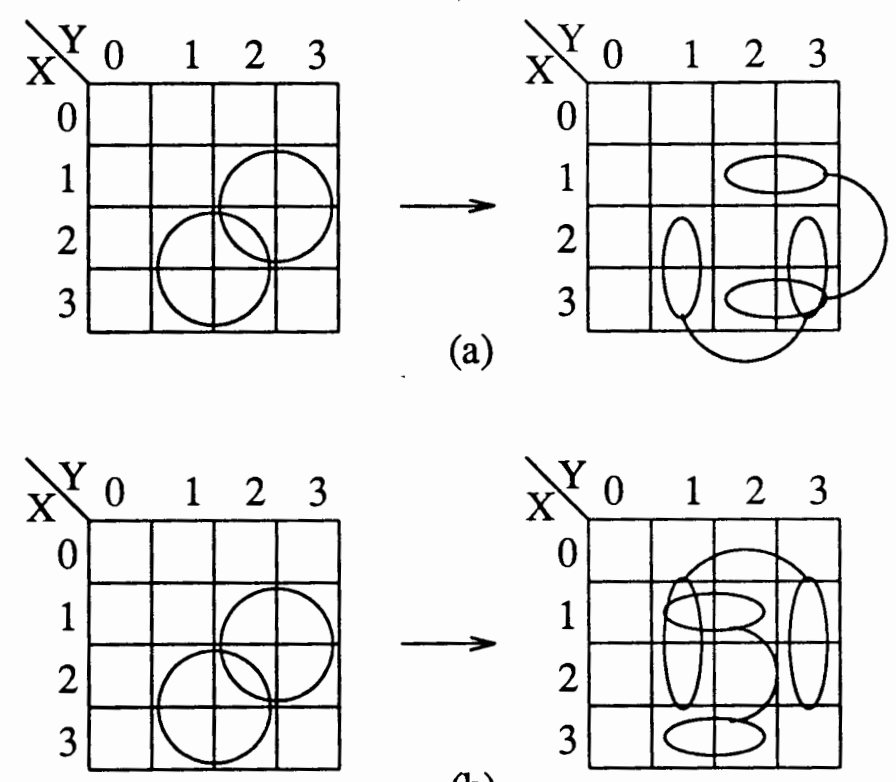

(b)

Figure 37. Difference 2 exorlinking $\left(S_{i} \backslash R_{i}, S_{j} \backslash R_{j}\right)$. as shown in Figure 37b. 
No compatible operation can be found in the previous literatrue.

The following two tables comparing the difference 2 operations of exorlinking with xlinking of EXORCISM [Perk 89] and operations of EXMIN [Sasa 90a].

TABLE VIII

COMPARISON OF EXORLINKING WITH XLINKING

\begin{tabular}{|c|c|c|c|c|c|c|c|}
\hline & $\begin{array}{l}S_{i} \cap R_{i}=\varnothing \\
S_{j} \cap R_{j}=\varnothing\end{array}$ & $\begin{array}{c}S_{i} \cap R_{i}=\varnothing \\
S_{j} \supset R_{j}\end{array}$ & $\begin{array}{c}S_{i} \cap R_{i}=\varnothing \\
S_{j} \backslash R_{j}\end{array}$ & $\begin{array}{l}S_{i} \supset R_{i} \\
S_{j} \supset R_{j}\end{array}$ & $\begin{array}{l}S_{i} \supset R_{i} \\
S_{j} \subset R_{j}\end{array}$ & $\begin{array}{l}S_{i} \supset R_{i} \\
S_{j} \backslash R_{j} \\
\end{array}$ & $\begin{array}{l}S_{S} \backslash R_{i} \\
\left.S_{j}\right) R_{j} \\
\end{array}$ \\
\hline$T_{S} \otimes T_{R}$ & $\begin{array}{c}\text { distance } 2 \\
\text { primary } \\
\text { xlinking }\end{array}$ & $\begin{array}{c}\text { distance } 1 \\
\text { secondary } \\
\text { xlinking }\end{array}$ & & $\begin{array}{l}\text { distance 1 } \\
\text { secondary } \\
\text { unlinking }\end{array}$ & & & \\
\hline$T_{R} \otimes T_{S}$ & $\begin{array}{c}\text { distance } 2 \\
\text { primary } \\
\text { xlinking }\end{array}$ & & & $\begin{array}{l}\text { distance 1 } \\
\text { secondary } \\
\text { unlinking }\end{array}$ & $\begin{array}{l}\text { distance } 2 \\
\text { primary } \\
\text { unlinking }\end{array}$ & & \\
\hline
\end{tabular}

TABLE IX

COMPARISON OF EXORLINKING WITH OPERATIONS IN EXMIN

\begin{tabular}{||c||c|c|c|c||}
\hline \hline & $S_{i} \cap R_{i}=\varnothing$ & $S_{i} \cap R_{i}=\varnothing$ & $S_{i} \cap R_{i}=\varnothing$ & $S_{i} \supset R_{i}$ \\
& $S_{j} \cap R_{j}=\varnothing$ & $S_{j} \supset R_{j}$ & $S_{j} \gamma R_{j}$ & $S_{j} \supset R_{j}$ \\
\hline \hline$T_{S} \otimes T_{R}$ & X-EXPAND-1 & RESHAPE & & X-REDUCE-2 \\
\hline$T_{R} \otimes T_{S}$ & X-EXPAND-1 & X-EXPAND-2 & & X-REDUCE-2 \\
\hline
\end{tabular}

\begin{tabular}{|l||c|c|c|}
\hline & $S_{i} \supset R_{i}$ & $S_{i} \supset R_{i}$ & $\left.S_{i}\right) R_{i}$ \\
& $S_{j} \subset R_{j}$ & $S_{j} \gamma R_{j}$ & $S_{j} \gamma R_{j}$ \\
\hline$T_{S} \otimes T_{R}$ & DUAL-COMPLEMENT & & \\
\hline$T_{R} \otimes T_{S}$ & X-REDUCE-1 & & \\
\hline
\end{tabular}

As we discussed in section III.3.3. (see page 33), difference 2 operations do not directly reduce the number of cubes in an ESOP. However, these operations provide opportunities for reducing the cost of ESOPs at later stages. Exorlinking has more difference 2 operations than approaches of EXMIN [Sasa 90a] and EXORCISM [Hell 88, Perk 89], which means it provides more opportunities for reducing the cost of ESOPs than 
EXMIN or EXORCISM.

Example III.20: Given is an ESOP with three terms: $T_{1}=X^{12} Y^{23}, T_{2}=X^{23} Y^{12}$ and $T_{3}=$ $X^{0} Y^{13}$. Any pairs of these three terms are different by two, but no operations in EXORCISM or EXMIN can be applied to these terms. We can, however, apply exorlinking to these terms. In Figure 38, three terms $T_{1}, T_{2}$ and $T_{3}$ are represented by three cubes $A, B$ and $C$, respectively. $A \otimes B$ generates $A^{\prime}$ and $B^{\prime} ; A^{\prime} \otimes C$ generates $A^{\prime \prime}$. The ESOP with three cubes is minimized to an ESOP with two cubes.

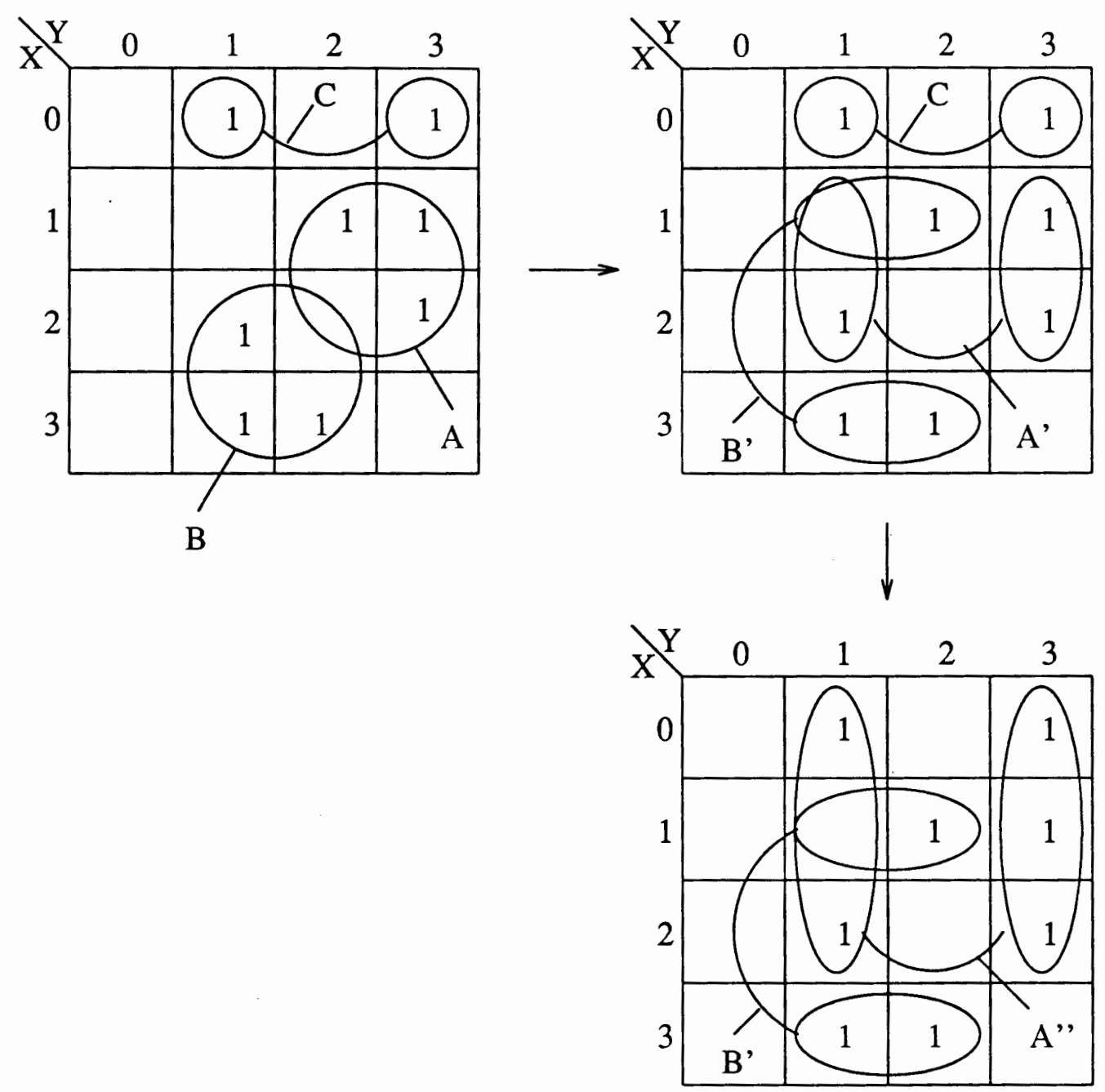

Figure 38. An example of ESOP minimization by difference 2 exorlinking. 


\section{IV.4. DIFFERENCE 3 EXORLINKING}

Difference 3 exorlink generates three resultant terms from two given terms. If $S_{i}$ $\supset R_{i}, S_{j} \cap R_{j}=\varnothing$, and $S_{k} \cap R_{k}=\varnothing$, the operation is equivalent to distance 2 secondary xlinking of EXORCISM [Perk 89].

Difference 3 exorlinking increases the number of terms in the ESOP. However, as we discussed in section III.3.4. (see page 34), increasing the number of terms may help to reduce the number of terms at the later stage and get better results.

Example III.21: In binary logic, a given ESOP with 4 cubes is as follows:

$$
000 x, 0 x 11, x 11 x, 1010 .
$$

The ESOP is shown in Figure 39a. Any pair of these cubes differ by 3. So, there are no difference 1 or difference 2 operations that can be performed. Performing difference 3 exorlinking on the first two cubes, we get:

$$
000 x \otimes 0 x 11=0111 \oplus 00 x 1 \oplus 0000 .
$$

Replacing the first two cubes by these three cubes, we get a new ESOP with five cubes:

$$
0111,00 x 1,0000, x 11 x, 1010,
$$

which is shown in Figure 39b. Two of the above cubes differ by 2:0000 and 1010. We can perform difference 2 exorlinking on them:

$$
0000 \otimes 1010=x 010 \oplus 00 x 0 .
$$

After this operation, the ESOP contains five cubes:

$$
0111,00 x 1, x 010, x 11 x, 00 \times 0,
$$

which is shown in Figure 39c.

Now, the cubes $00 x 1$ and $00 \times 0$ differ by 1 , we can perform difference 1 exorlinking on them:

$$
00 x 1 \otimes 00 x 0=00 x x .
$$

The ESOP now contains four cubes: 


$$
0111,00 x x, x 010, x 11 x
$$

which is shown in Figure 39d.

Performing difference 2 exor linking on cubes $x 010$ and $x 11 x$, we obtain:

$$
x 010 \otimes x 11 x=x x 10 \oplus x 111 .
$$

The ESOP is

$$
0111,00 x x, x x 10, x 111
$$

which is shown in Figure 39e.

Cubes 0111 and $x 111$ can be combined to one cube:

$$
0111 \otimes x 111=1111
$$

The final result is an ESOP with three cubes as shown in Figure 39f. By using difference 3 exorlinking, the number of the ESOP is temporarily increased, but it helps to jump out of the local minimum and achieve a better result.

\section{IV.5. SUMMARY}

Xlinking uses one formula to present the primary xlinking for any distances. Another formula is used for distance 2 secondary xlinking. Separate rules are used for unlinking. There are no general formulas in EXMIN. Each operation is presented by a separate formula. However, there are more operations in difference 1 and difference 2 in EXMIN than in EXORCISM. That is one of the reasons that EXMIN generates better results than EXORCISM [Sasa 90a]. Exorlinking is described by one formula. It contains all the possible operations in xlinking approach. In other words, it can link any two terms in a given ESOP without condition. All the operations used in EXORCISM and EXMIN are included in exorlinking as special cases. In xlinking approach, the basic method is to perform different cube operations iteratively to combine or reshape cubes. More operations will provide more opportunity to minimize the ESOPs. This is the reason that exorlinking is superior than xlinking and the operations in EXMIN. 
$\mathrm{X} 3 \mathrm{X} 4$

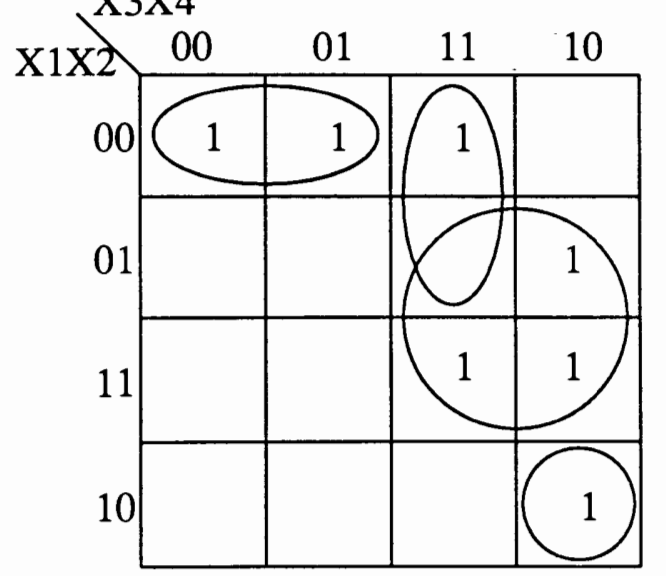

(a)

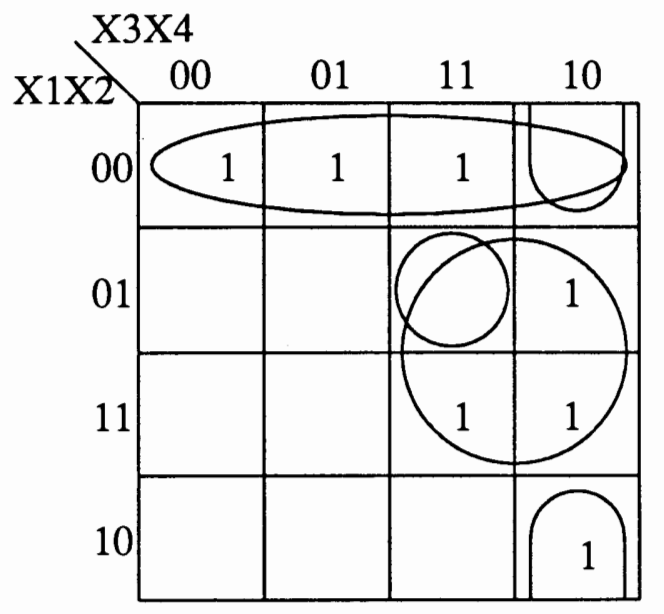

(d)

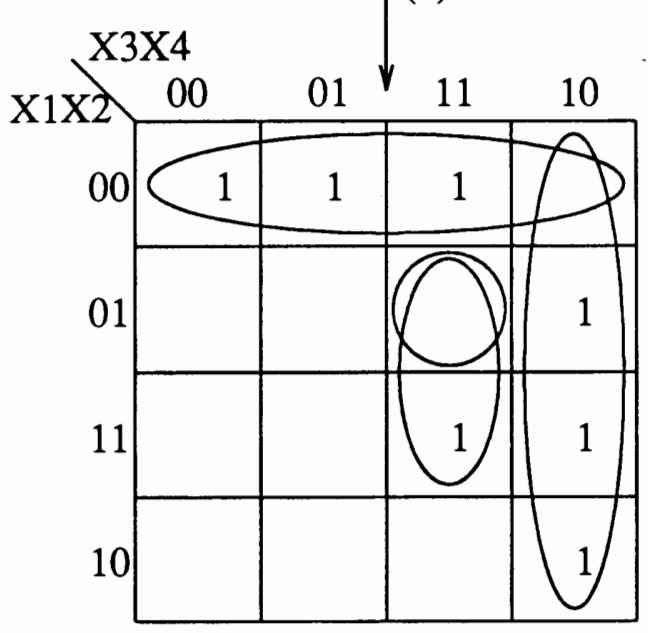

(e)

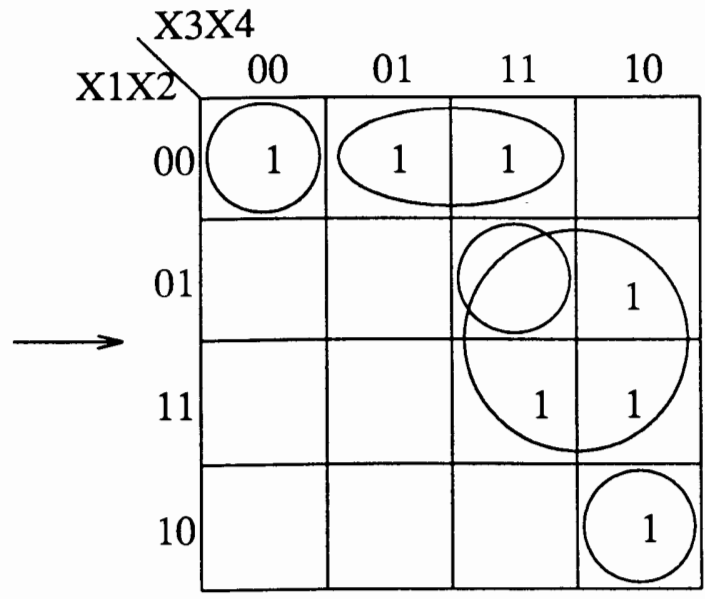

(b)

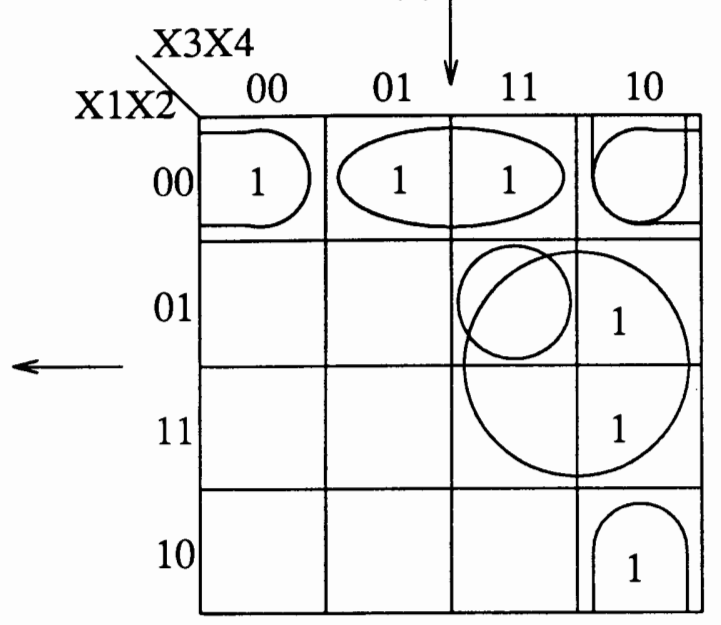

(c)

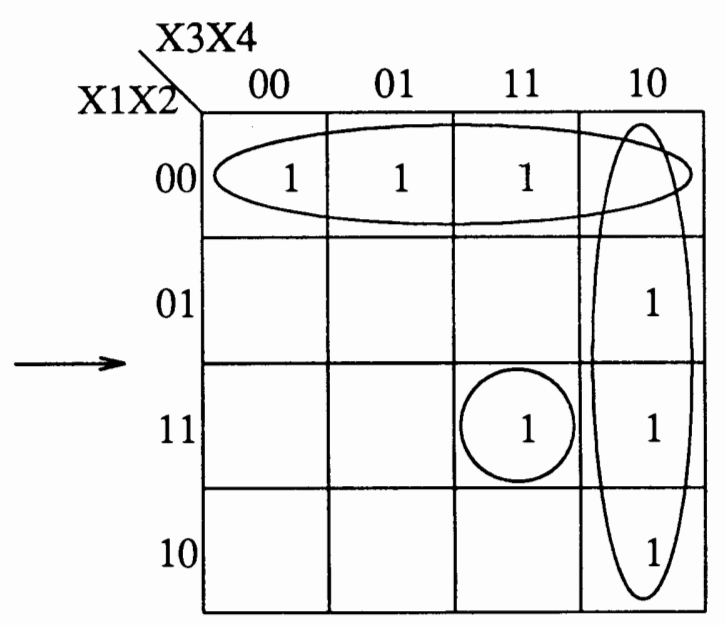

(f)

Figure 39. An example of ESOP minimization by difference 3 exorlinking. 


\section{CHAPTER V}

\section{ALGORITHM OF EXORCISM-MV-2 PROGRAM}

\section{V.1. THE ALGORITHM OF EXORCISM}

EXORCISM uses the following algorithm to minimize an ESOP:

1. For each pair of cubes in the ESOP, do distance 1 primary xlinking.

2. For each pair of cubes in the ESOP, do distance 2 primary xlinking.

3. For each pair of cubes in the ESOP, do distance 1 secondary xlinking.

4. For each pair of cubes in the ESOP, do distance 2 secondary xlinking.

5. If the cost is reduced, go to step 1 , else go to step 6 .

6. Do unlinking.

7. If time has not exceeded the time limit, then go to step 1, else stop.

\section{V.2. THE ALGORITHM OF EXMIN}

1. For each pair of cubes in the ESOP, do X-MERGE if possible.

2. For each pair of cubes in the ESOP, do the following operations if possible: RESHAPE, DUAL-COMPLEMENT, X-EXPAND-1, AND X-EXPAND-2.

3. For the cubes modified by the above operations, do X-MERGE if possible.

4. In step 3, if X-MERGE is performed, go to step 2, else go to step 5.

5. In step 3, if X-EXPAND-1 or X-EXPAND-2 is performed, go to step 2, else go to step 6. 
6. For each pair of cubes in the ESOP, do X-REDUCE-1 and X-REDUCE-2 if possible.

7. If the number of cubes is reduced, go to step 1, else stop.

While EXMIN has more operations for a difference 2 pair of cubes than EXORCISM has, it also introduces a new problem: it may fall into an infinite loop. Sasao gave the following example to show such a case.

Example III.22:

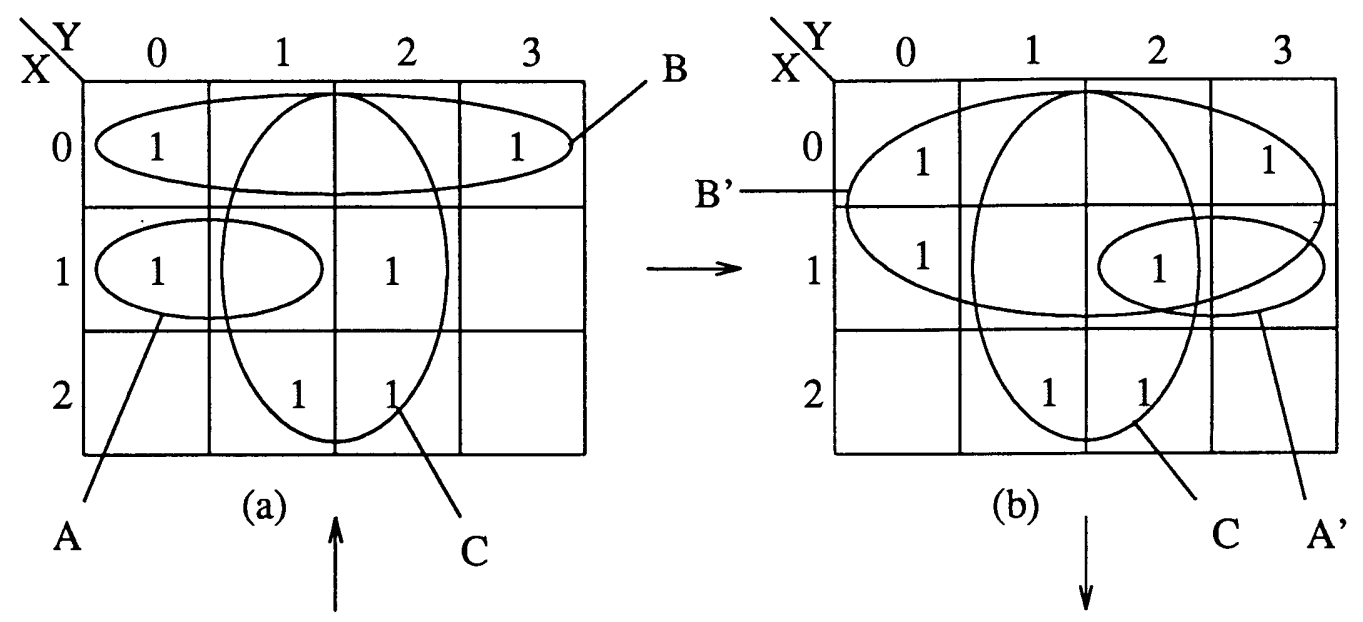

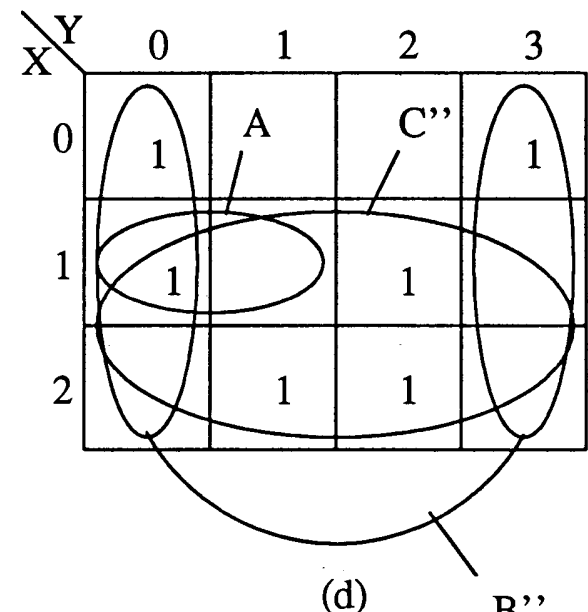

(d)

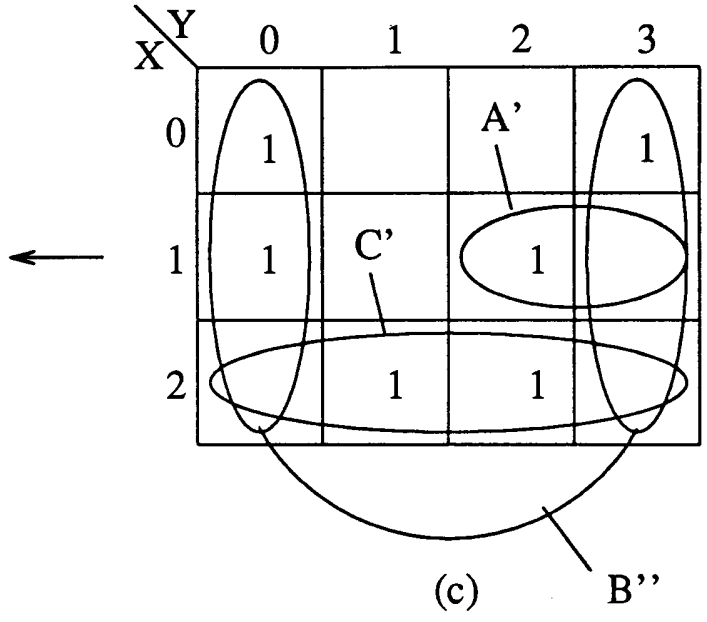

Figure 40. An example of infinite loop in EXMIN.

In Example III.22, an ESOP with three cubes $A=[010-1100], B=[100-1111]$ and $C$ 
$=[111-0110]$ is shown in Figure 40a. Performing X-EXPAND-2 on cubes $A$ and $B$, we get $A^{\prime}$ and $B^{\prime}$ in Figure 40b. Performing DUAL-COMPLEMENT on $B^{\prime}$ and $C$, we get $B^{\prime \prime}$ and $C^{\prime}$ as shown in Figure 40c. Performing X-EXPAND-2 on cubes $A^{\prime}$ and $C^{\prime}$, we get cube $A$ and $C^{\prime \prime}$ as shown in Figure 40d. Performing DUAL-COMPLEMENT on $B^{\prime \prime}$ and $C^{\prime \prime}$, we get cubes $B$ and $C$, which is the original ESOP as shown in Figure 40a.

For avoiding the problem of infinite loop, Sasao separates the difference 2 operations to two groups: X-REDUCE-1 and X-REDUCE-2 form one group, and the rest of the operations form another group. Two groups of operations are performed separately (step 2 and step 6 in above).

\section{V.3. THE NEW ALGORITHM}

As we discussed in section 4.3 , the main purpose of difference 2 operations is to provide opportunities for difference 1 or difference 0 operations. So, the new algorithm is: instead of doing all possible difference 2 operations, perform only those difference 2 operations which will lead to difference 0 or difference 1 operations. More specifically, if two cubes, $A$ and $B$, are different by 2 , then $A \otimes B$ generates resultant cubes $C_{1}$ and $C_{2}$, $B \otimes A$ generates resultant cubes $D_{1}$ and $D_{2}$. We check the cubes $C_{1}, C_{2}, D_{1}$, and $D_{2}$ with all the cubes in the ESOP except cubes $A$ and $B$. If difference 0 or difference 1 operations are possible, we perform the difference 2 operation $A \otimes B$ or $B \otimes A$ followed by difference 0 or difference 1 operations. Otherwise, this difference 2 operation is not performed.

Example III.23: Given an ESOP with 5 cubes:

$$
001101101111010 \times 10 \times 1 .
$$

If we perform all the possible difference 2 operations on these cubes, the procedure is shown in Figure 41. 


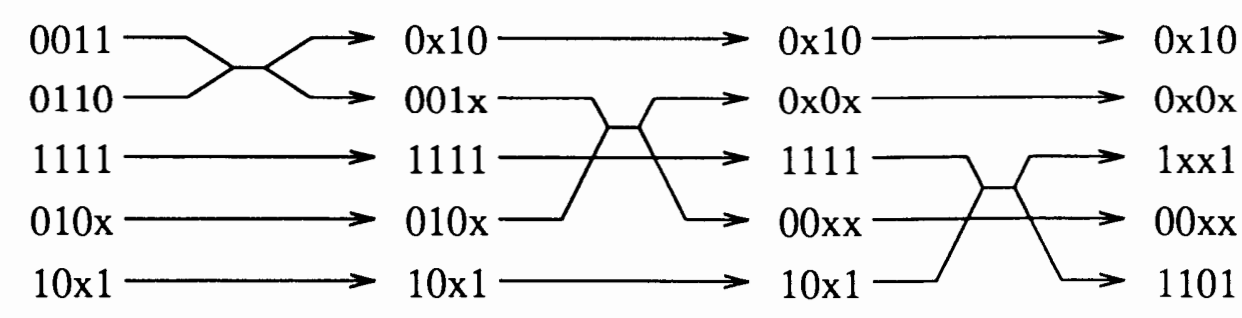

(a)

(b)

(c)

(d)

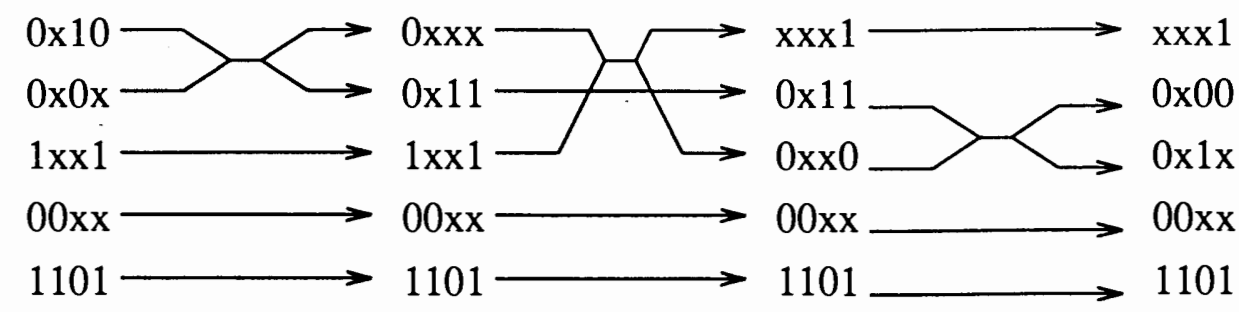

(d)

(e)

(f)

$(\mathrm{g})$

Figure 41. An example of performing all possible difference 2 operations.

Figure 42 shows the procedure using Karnaugh maps. From one map to the next map, two cubes are reshaped. Figure 42a shows the original ESOP with 5 cubes. After six operations, the same function is represented by another ESOP with 5 cubes as shown in Figure 42g. The number of cubes has not been reduced so far.

Our new approach is shown in Figure 43:

1. Perform all possible remove_equal and difference 1 exorlinking operations. In this example, none of these operations are possible now (see Figure 43a).

2. Check if two cubes differ by 2 . For instance, cube 0011 and 0110 differ by 2 as shown in Figure 43a.

3. Check the two pairs of resultant cubes with other cubes in the array to see if we can find two cubes that differ by 0 or by 1 . In the example, cube 0011 and 0110 generate two pairs of resultant cubes:

$$
0110 \otimes 0011=0 x 11 \oplus 011 x
$$

as shown in Figure 43b1, and 


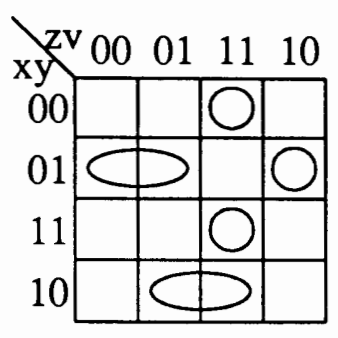

(a)

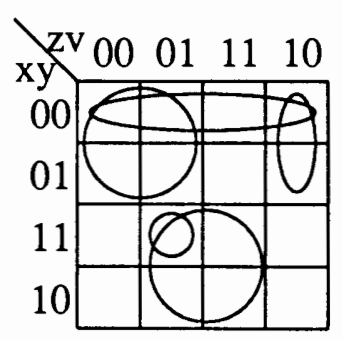

(d)

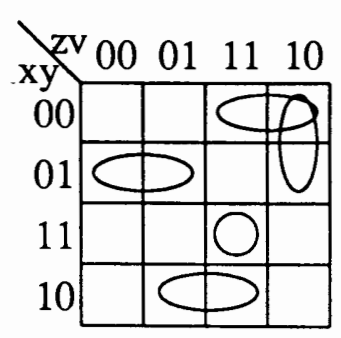

(b)

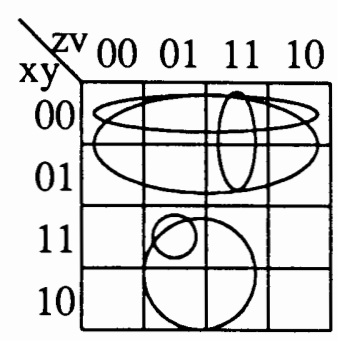

(e)

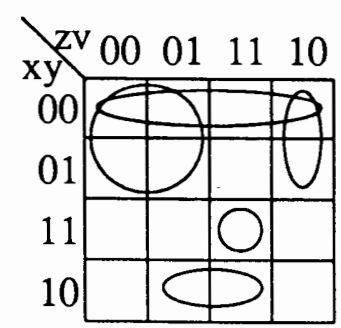

(c)

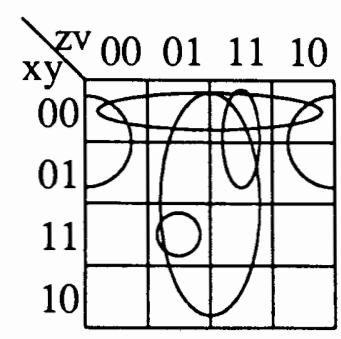

(f)

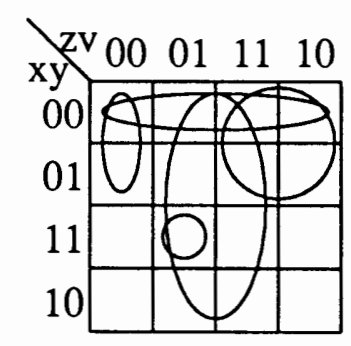

(g)

Figure 42. Karnaugh maps for Example III.23.

$$
0011 \otimes 0110=0 x 10 \oplus 001 x
$$

as shown in Figure $43 \mathrm{~b} 2$.

We check these four resultant cubes with the rest of the cubes in the array:

\section{$1111010 x 10 \times 1$}

and we can find that cubes $011 x$ and $010 x$ are different by 1 as shown in Figure $43 \mathrm{~b} 1$.

4. In step 3, if we can find two cubes that differ by 0 or different by 1 , we perform the difference 2 exorlinking and then perform the remove_equal or difference 1 exorlinking operation. For instance, in step 3 we found two cubes $011 x$ and $010 x$ that 

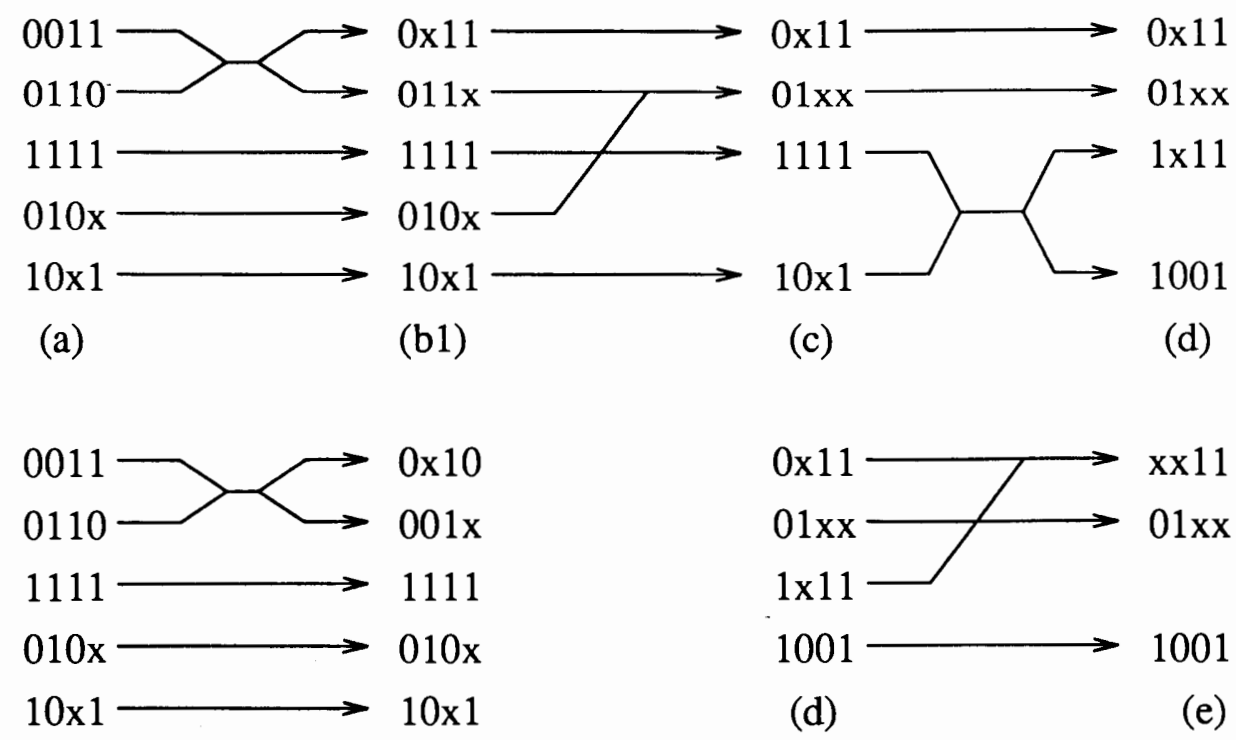

(a) (b2)

Figure 43. Conditionally performing difference 2 exorlinking.

differ by 1 , we perform

$$
0110 \otimes 0011=0 x 11 \oplus 011 x
$$

and then we perform

$$
011 x \otimes 010 x=01 x x
$$

as shown in Figure 43c.

After performing remove_equal or difference 1 exorlinking, we go back to step 1 . If we can not find any two cubes that differ by 0 or by 1 in step 3 , we do not perform difference 2 operation, and go back to step 2 to check other two cubes.

We continue this loop as shown in Figure 43d and 43e. If the number of cubes has not been reduced for certain number of iterations, we go to next stage of minimization. Figure 44 shows this approach in the Karnaugh maps. Comparing the Figure 41 with the Figure 43, we can see that our new approach is more efficient. 


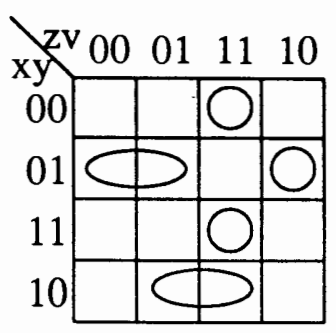

(a)

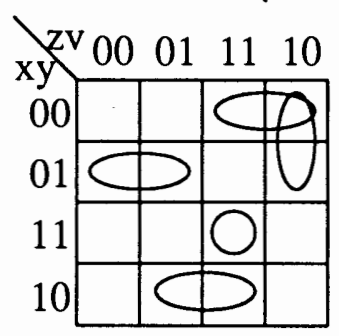

(b1)

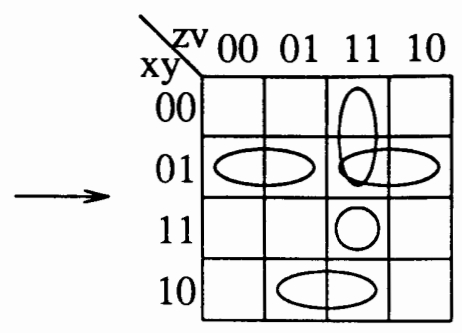

(b2)

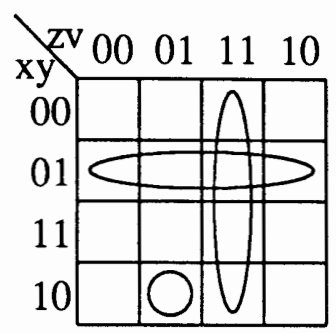

(e)

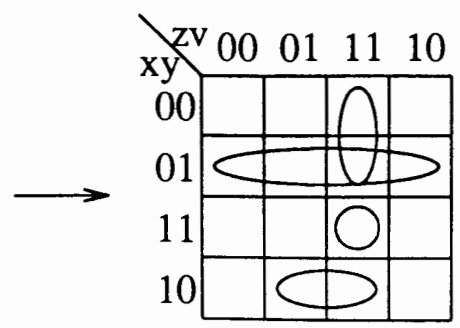

(c)

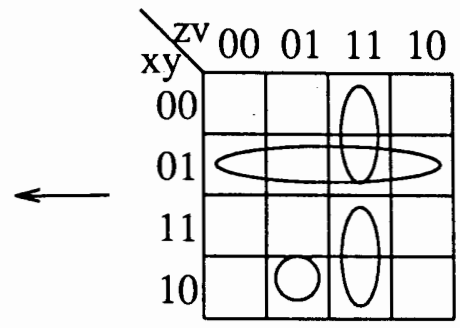

(d)

Figure 44. Karnaugh maps corresponding to Figure 43.

\section{V.4. MINIMIZATION OF MULTIPLE OUTPUT FUNCTIONS}

There are two ways to minimize a multiple output function:

1. decompose the multiple output functions to single output functions, minimize each single output function separately, and then minimize the set of functions again;

2. minimize the multiple output function directly.

Both the EXORCISM and the EXMIN use the first method. In our program, we let the user to select which method to use. The following procedure shows our algorithm.

1. If the function is a multiple output one and the option "decomposition to single output" is selected, then go to step 2; otherwise, go to step 5 .

2. Decompose the function to a set of single output functions.

3. Minimize each single output function separately. 
4. Combine the minimized single output functions to a multiple output function.

5. Minimize the function.

Example III.24 shows this procedure.

Example III.24: In Example II.11 (page 22), the multiple-output function $F(x, y, z)$ can be represented by the following array of cubes:

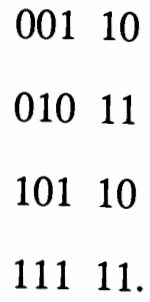

The first three symbols in each cube represent input variables, the last two symbols represent output variables. For instance, the first cube in the array, 00110 , means that when input combination is $x=0, y=0$, and $z=1$, the output variables $f_{0}=1$ and $f_{1}=$ 0 . Since this is a two output function, we can decompose it to two single output functions which is represented by the following array of 6 cubes:

\section{0 \\ 01010 \\ 10110 \\ 11110 \\ 01001}

11101.

By minimizing the first 4 cubes, we get

$$
\begin{aligned}
& 01 x \quad 10 \\
& x x 110 .
\end{aligned}
$$

By minimizing the last two cubes, we get 
Put these 4 cubes together, we get a solution, which is

$$
\begin{array}{ll}
01 x & 10 \\
x x & 10 \\
010 & 01 \\
111 & 01 .
\end{array}
$$

Minimize these 4 cubes again, we get the final result:

$$
\begin{aligned}
& \begin{array}{lll}
x & 11 & 01
\end{array} \\
& x x 110 \text {. } \\
& 01 x \quad 11
\end{aligned}
$$

which is the same result we showed in Example II.11.

By experimentation, we found that this method is on average better than the method without decomposition. Table $\mathrm{X}$ shows the comparison. In our algorithm, by default, the decomposition to a set of single output functions is used.

TABLE X

\section{MULTIPLE OUTPUT FUNCTION}

\begin{tabular}{||l||l|l|l|l||l|l|l|l||}
\hline \hline \multicolumn{1}{|c||}{} & \multicolumn{3}{c|}{ Decomposition to single output functions } & \multicolumn{3}{c|}{ Without decomposition } \\
\cline { 2 - 9 } & cube & AND & EXOR & Time & cube & AND & EXOR & Time \\
\hline \hline ADR4 & 31 & 114 & 40 & 7.5 & 31 & 106 & 40 & 5.4 \\
\hline LOG8 & 95 & 508 & 192 & 266.8 & 96 & 515 & 226 & 207.4 \\
\hline MLP4 & 62 & 301 & 86 & 38.2 & 62 & 311 & 96 & 43.8 \\
\hline NRM4 & 67 & 375 & 131 & 51.1 & 72 & 404 & 136 & 65.0 \\
\hline RDM8 & 31 & 110 & 42 & 8.5 & 31 & 111 & 44 & 10.7 \\
\hline ROT8 & 37 & 200 & 62 & 6.4 & 36 & 193 & 64 & 8.2 \\
\hline SQR8 & 114 & 533 & 202 & 264.4 & 126 & 622 & 240 & 352.4 \\
\hline WGT8 & 58 & 261 & 64 & 60.5 & 60 & 260 & 79 & 250.2 \\
\hline
\end{tabular}

In Table X, cube indicates how many cubes (terms) in the solution. AND indicates how many wires to AND gates. EXOR indicates how many wires to the EXOR gates. Time indicates the user time on a SPARC II station. 


\section{V.5. MINIMIZATION OF INCOMPLETELY SPECIFIED FUNCTIONS}

An incompletely specified function can be represented by an $\mathrm{ON}$-array of cubes and a DC-array of cubes. We can minimize an incompletely specified function by minimizing its $\mathrm{ON}$-array of cubes only. However, linking the $\mathrm{ON}$-array of cubes with the DCarray of cubes may generate better results.

Example III.25: Given is an ESOP with one ON-cube, $01 \times 1$, and two DC-cubes, $11 \times 1$ and $1 \times 10$, as shown in Figure 45a. The function can be represented by the ON-cube only. By linking the ON-cube with one of the DC-cubes, we get the cube $x 1 x 1$ as shown in Figure 45b, which is a better result than ON-cube $01 x 1$.

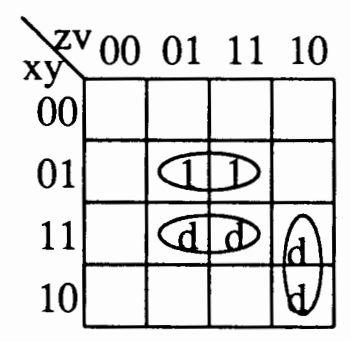

(a)

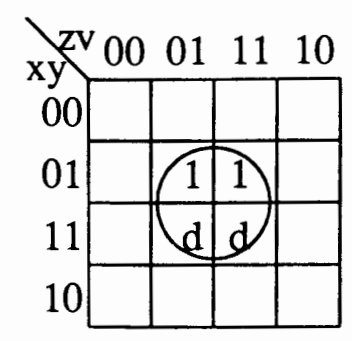

(b)

Figure 45. Example of minimizing an incompletely specified function.

Saul [Saul 90] pointed out that minimization of incompletely specified functions in ESOP form is difficult, because:

1. The DC-cubes may cover some minterms which are not in the DC-array, as shown in Figure 46.

2. The DC-array may not contain a cube that can be directly linked with a cube in ON-array because of positions or sizes of the DC-cubes, as shown in Figure 47a and 48a, respectively.

In Figure 46, cubes $x 101$ and $01 x 1$ are in the DC-array, and cube $x 100$ is an ONcube. We can not link the DC-cube $x 101$ with the ON-cube $x 100$, because the DC-cube 


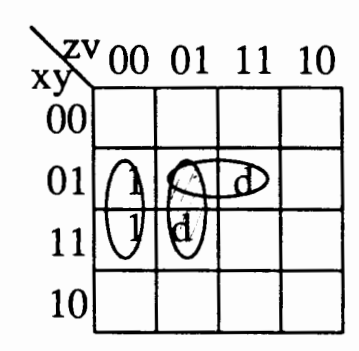

Figure 46. Linking DC-cubes.

$x 101$ contains a minterm 0101, which is a false minterm. This problem can be solved by making the DC-array disjoint. In a disjoint DC-array, each DC-minterm is covered by a cube once, and a false minterm is not covered by any cubes.

In Figure 47a, the ON-cube can not be linked with any one of the DC-cubes. If the DC-cubes are in the right position, however, they can be linked as shown in Figure $47 \mathrm{~b}$ and $47 \mathrm{c}$.

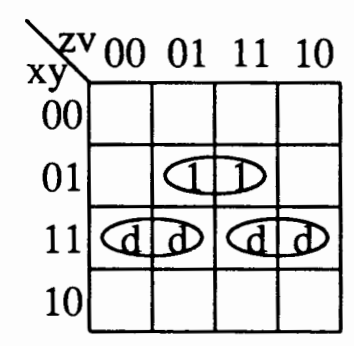

(a)

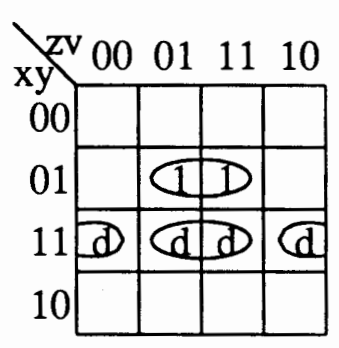

(b)

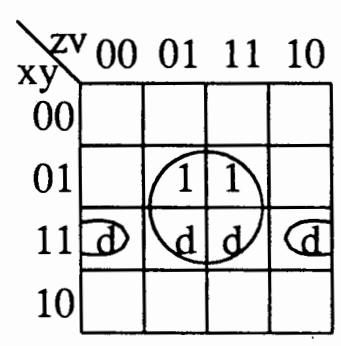

(c)

Figure 47. The position of DC-cubes.

In Figure 48a, the ON-cube can not be linked with the DC-cube, because the size of the DC-cube is larger than the size of the ON-cube. If we can separate the DC-cube properly, as shown in Figure 48b, then the ON-cube can be linked with one of the DCcubes, as shown in Figure 48c.

Saul [Saul 90] gave the following algorithm to link the ON-cubes with the DCcubes: 


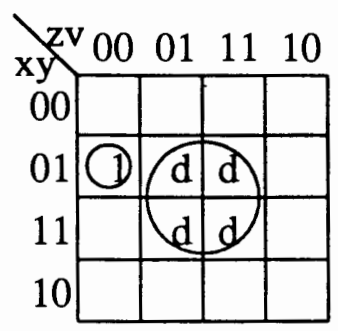

(a)

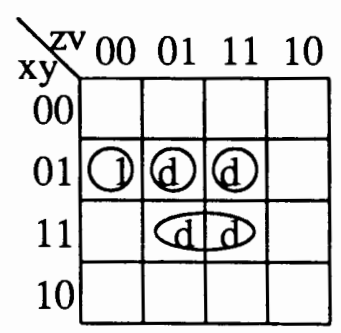

(b)

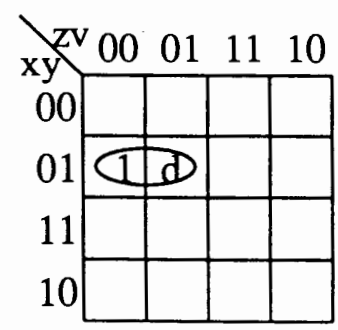

(c)

Figure 48. The size of DC-cubes.

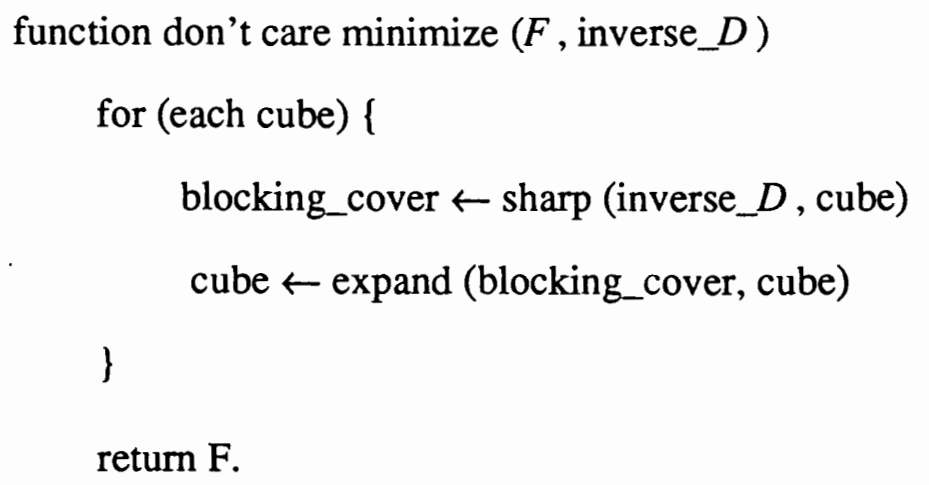

Here, $F$ is the $\mathrm{ON}$-array of cubes, $D$ is the DC-array of cubes, and inverse $D D$ is the complemented DC-array of cubes. Before calling this algorithm, the DC-array is converted to the disjoint sum of products representation, and then complemented. In the algorithm, "for each cube" means for each cube in the ON-array $F$. Inverse_ $D$ covers all the minterms that are not covered by the DC-array. Please note that inverse $D$ is an array of cubes. So, sharp(inverse_ $D$, cube) means

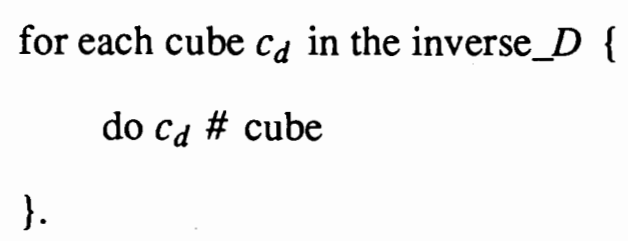

By using the sharp operation, block_cover contains the minterms that are not covered either by DC-array or by the cube. The operation expand is carried out by expanding each cube in such a way that it does not intersect the cube with the blocking_cover. Let us look at the following example to make this procedure clear. 
Example III.26: Given an ON-array with one cube, and a DC-array with two cubes as shown in Figure 49a.

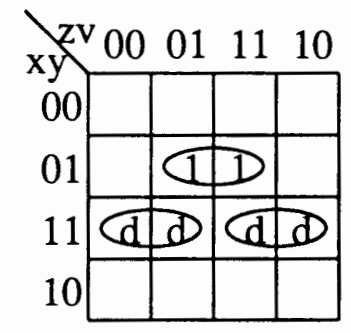

(a)

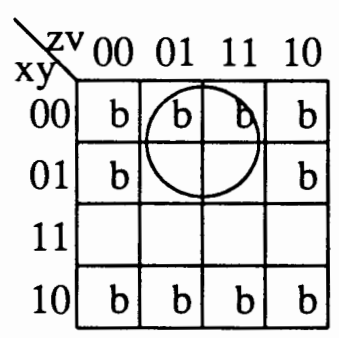

(d)

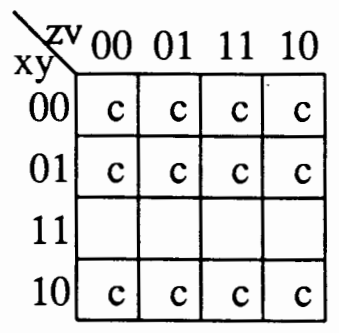

(b)

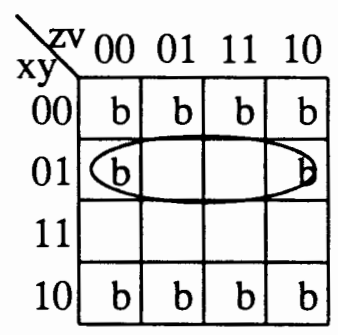

(e)

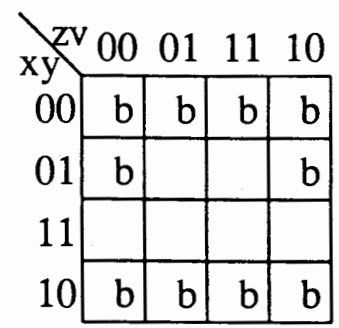

(c)

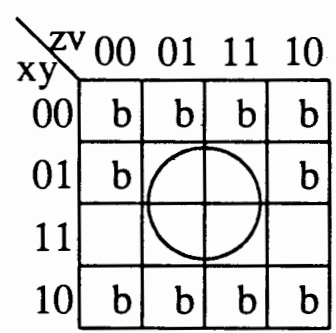

(f)

Figure 49. Linking DC-cubes by Saul's algorithm.

The two DC-cubes can not be linked with the ON-cube because of their positions. According to Saul's algorithm, the following operations will be performed:

1. Generate inverse_ $D$ : Inverse_ $D$ is an array of cubes which should cover all the minterms marked as "c" in Figure 49b. Inverse_ $D$ can be in the form of disjoint SOPs or non-disjoint SOPs.

2. Generate block_cover: Performing the sharp operation on inverse $\_D$ and the ONcube, we can get an array of cubes called block_cover. Block_cover contains all the minterms marked as "b" in Figure 49c. These minterms are not covered by DC-cubes and are not covered by the ON-cube.

3. Expanding the ON-cube: There are three ways to expand the ON-cube $01 \times 1$ as shown in Figure 49d, 49e and 49f, respectively. In Figure 49d and 49e, the 
expanded ON-cube intersects the block_cover, which means such an expanding is impossible. So, the only possible way to expand the ON-cube is shown in Figure $49 \mathrm{f}$.

Saul's algorithm has an obvious limitation: it tries to reduce the number of connections but does not consider the possibility of reducing the number of cubes. There are two possible ways to reduce the number of cubes:

1. Remove an ON-cube if it is equal to a DC-cube.

2. Delete an ON-cube if it is contained by a DC-cube.

The following two examples show the above two cases respectively.

Example III.27: Given is an ON-array of three cubes

0101

0111

1101

and a DC-array of one cube

\section{1}

as shown in Figure 50a. After minimizing the ON-array, we get two ON-cubes $x 1 \times 1$ and 1111 (Figure 50b). By comparing the ON-cubes with the DC-cube (Figure 50c), we find out that the ON-cube 1111 is equal to the DC-cube 1111 . We remove these two cubes, and get the result $x 1 \times 1$ which is one cube less than the minimized ON-array (Figure $50 \mathrm{~d})$.

Example III.28: Given is the same ON-array as in Example III.27, and a DC-array of one cube $111 x$ as shown in Figure 51a. After minimizing the ON-cubes as shown in Figure 51b, we can find out that the DC-cube $111 x$ contains the ON-cube 1111 as shown in Figure 51c. We delete the ON-cube 1111. The result is shown in Figure 51d, which is the same as Example III.27: 


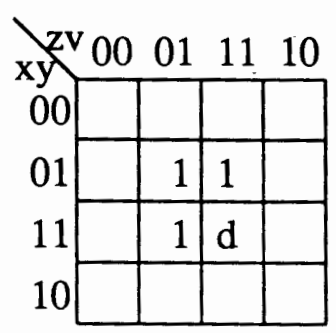

(a)

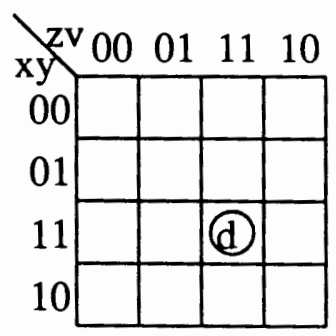

(c)

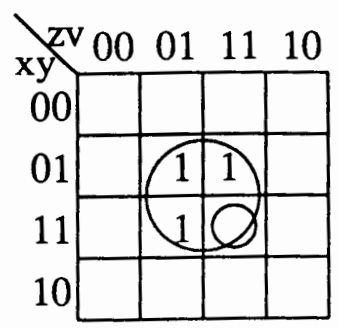

(b)

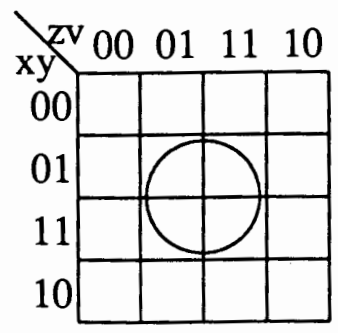

(d)

Figure 50. An ON-cube is equal to a DC-cube.

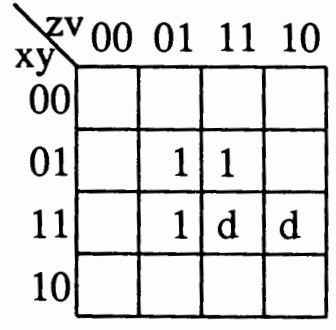

(a)

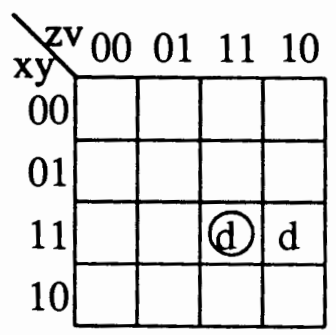

(c)

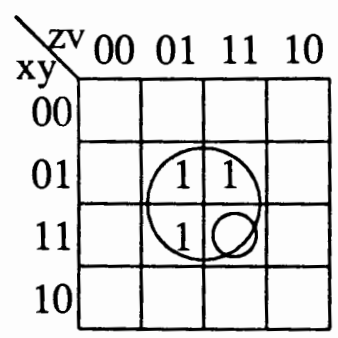

(b)

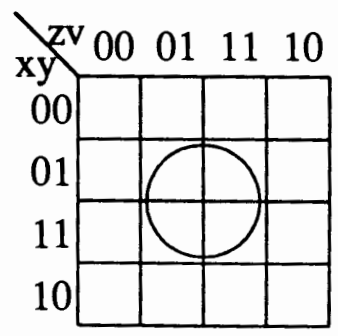

(d)

Figure 51. An ON-cube is contained by a DC-cube. 
To check whether a DC-cube contains or is equal to an ON-cube, we use the disjoint sharp operation:

$$
\mathrm{R} \leftarrow \text { ON-cube \# } \#_{d} \text { DC-cube. }
$$

If the sharp operation returns an empty cube, this means that the DC-cube contains the ON-cube, or is equal to it. The ON-cube can then be removed.

Based on the above discussion, our approach to minimize incompletely specified functions is described by the following procedure:

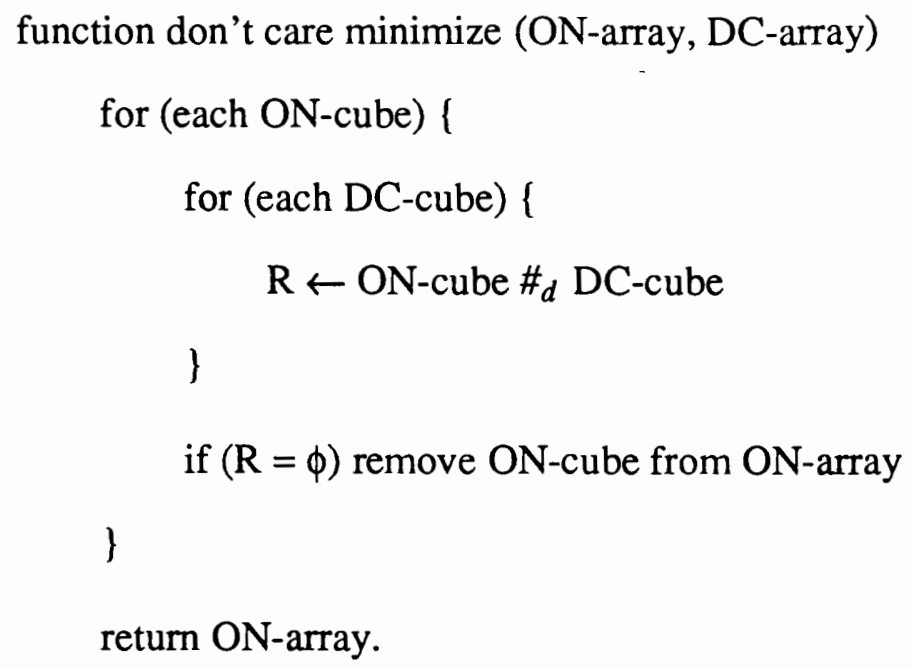

If no more ON-cubes can be removed by this method, we can perform difference 2 exorlinking on the ON-array in order to reshape the ON-cubes. Example III.29 shows that reshape may help to reduce the $\mathrm{ON}$-cubes.

Example III.29: Given ON-cubes

and DC-cubes

as shown in Figure 52a. The minimization is carried out by the following steps: 


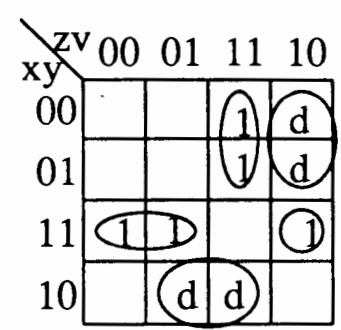

(a)

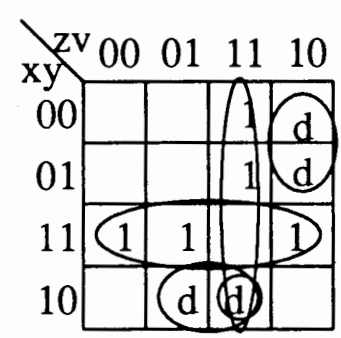

(c)

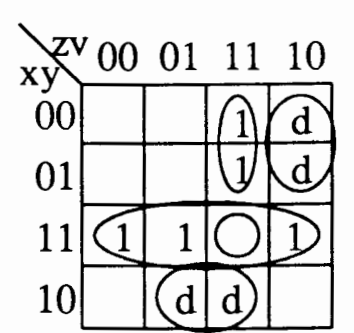

(b)

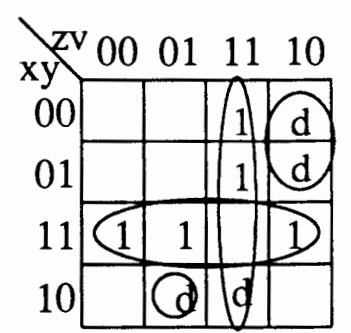

(d)

Figure 52. Minimization of an incompletely specified function.

1. Perform the don't care minimization procedure. None of the ON-cubes can be removed.

2. Reshape the ON-cubes as shown in Figure 52b. Again, none of the ON-cubes can be removed.

3. Reshape the ON-cubes as shown in Figure 52c, we get three ON-cubes:

$$
\begin{aligned}
& 11 x x \\
& x x 11 \\
& 1011 .
\end{aligned}
$$

Since the DC-cube $10 \times 1$ contains the ON-cube 1011 , the operation

$$
1011 \#_{d} 10 \times 1
$$

generates an empty cube. So, the ON-cube 1011 can be removed. The final result is an array of two cubes: $11 x x$ and $x x 11$.

By performing sharp and difference 2 exorlinking iteratively, the number of $\mathrm{ON}$ - 
cubes can be reduced, which serves our primary goal: minimizing the number of terms in the ESOPs. After we tried all possible reshapes, the next step is to achieve our secondary goal: minimizing the number of connections. This is done by trying all possible expanding operations of ON-cubes, as shown in Figure 49.

The next section presents our whole algorithm.

\section{V.6. THE ALGORITHM OF EXORCISM-MV-2}

We take the disjoint cubes as our starting point. The pairs of equal cubes are removed and difference 1 exorlinking operations are performed iteratively. Then difference 2 exorlinking operations are executed which may provide opportunities for difference 1 exorlinking. If difference 2 exorlinking can not further improve the cost function, difference 3 exorlinking is performed. After certain loops, if no further difference 1 exorlinking operations are possible, difference 2 exorlinking operations are performed to minimize the number of connections.

In the case of a multi-output function, by default, the function is first transformed from a multiple-output array to single-output arrays. We minimize each single-output array, and then minimize the whole function, by using the methods discussed in section V.4.

For incompletely specified functions, the ON-array is minimized first. Then the disjoint sharp operation is executed between each cube in the $\mathrm{ON}$-array and the cubes in the DC-array. If the disjoint sharp operation generates an empty cube, the ON-cube will be removed from the $\mathrm{ON}$-array. If no more cubes in the $\mathrm{ON}$-array can be sharped out, difference 2 exorlinking operations are performed in order to provide further opportunities. Next, we try to expand each ON-cube into DC-cubes. Successful application of these operations decreases the numbers of connections. 
Since our algorithm is a heurist one, we do not know whether or not we have got the minimum results. So, we need some criteria to stop the program. The following methods can be used as termination criteria:

1. Cost functions. We can use a cost function as a termination criteron. For instance, we can stop the program if the number of terms in the current solution meets a preset number.

2. Number of loops. We can stop the program after a certain number of loops. This is a simple method. But the quality of the results is not guaranteed.

3. Execution time. We can stop the program if time limit has been exceeded. This is the method used in EXORCISM.

4. Improvements of the current solution. By this method, the program is controlled by comparing the current result with the previous result. If no improvement for a certain number of loops, the program goes to the next step. This is the method used in ESPRESSO and EXMIN. In our program this is the method used by default. The user can also select other methods as options.

Algorithm to Minimize MIESOP Expressions for Incompletely Specified Functions.

Input: ON-array and DC-array of disjoint cubes for a multi-valued input function.

1. $F:=O N ; D:=D C$.

2. SOLUTION $:=\mathrm{F}$, MIN_COST $:=\operatorname{COST}(\mathrm{F})$. (MIN_COST will be updated in the steps below to reflect always the lowest cost of solutions obtained until now. This solution is also stored).

3. If the option "do not decompose the function to single output function" is selected, go to step 5; else go to step 4 .

4. Decompose the function to a set of single output functions. For each single output function, perform the steps 5 to 8 . 
5. Perform all possible remove_equal operations.

6. Perform all possible difference 1 exorlinking operations.

7. For each pair of cubes in ESOP, check if a difference 2 exorlink is possible. If it is possible, further check if it makes a remove_equal operation or a difference 1 exorlink operation possible. If it is possible, perform the difference 2 exorlink operation and then perform remove_equal or difference 1 exorlink operation. Otherwise, do nothing.

8. Check the number of cubes. If the number of cubes has not been reduced for certain number of iterations go to 9 , else go to 5 .

9. If the option "do not decompose the function to single output function" is selected, go to step 11 .

If the single functions have been combined to a multiple output function, go to step 11.

If all the single output functions are minimized, go to step 10 ;

else go to step 5 to minimize the next single output function.

10. Combine the single output functions to a multiple output function.

11. Perform difference 3 exorlink operation iteratively.

12. Check the number of cubes. If the number of cubes has not been reduced for certain number of iterations go to 13 , else go to 5 .

13. Check all possible difference 2 exorlinking operations. For each difference 2 exorlink operation, if it reduces the cost, perform the difference 2 exorlink operation, otherwise, do nothing.

14. If the option "don't care" is not selected, go to 21, else go to 15.

15. If $\mathrm{D}$ is empty (no dc cubes) go to 21 , else go to 16 . 
16. Perform disjoint sharp between each cube in $\mathrm{F}$ and all cubes in $\mathrm{D}$.

17. Minimize $\mathrm{F}$ again (perform the steps from 5 to 11 )

18. If the number of cubes has not been reduced for certain number of iterations, go to 19 , else goto 15 .

19. Expand each cube in $\mathrm{F}$ into $\mathrm{D}$ if possible.

20. Check the cost. If the cost has no improvement for certain number of iterations go to 21 , else go to 19 .

21. Print the output and stop the program. 


\section{CHAPTER VI}

\section{EVALUATION OF RESULTS OF EXORCISM-MV-2}

Exorcism-mv-2 was tested on a set of MCNC benchmarks, as illustrated in Tables XI, XII, XIII. All the benchmarks are run on the Sparc II station. The time is the user time in seconds.

TABLE XI

EXPERIMENTAL RESULTS OF FUNCTIONS WITH 1 BIT AND 2 BIT DECODERS

\begin{tabular}{||l||l|l|l|l||l|l|l|l||}
\hline \hline \multicolumn{1}{|c||}{} & \multicolumn{4}{c||}{ bit decoder } & \multicolumn{4}{c|}{ 2 bit decoder } \\
\cline { 2 - 9 } & cube & AND & EXOR & Time & cube & AND & EXOR & Time \\
\hline \hline ADR4 & 31 & 114 & 40 & 4.5 & 11 & 46 & 14 & 4.7 \\
\hline LOG8 & 95 & 508 & 192 & 266.8 & 85 & 663 & 163 & 500.3 \\
\hline MLP4 & 62 & 301 & 86 & 38.2 & 51 & 380 & 75 & 89.6 \\
\hline NRM4 & 67 & 375 & 131 & 51.1 & 53 & 429 & 86 & 66.8 \\
\hline RDM8 & 31 & 110 & 42 & 8.5 & 26 & 152 & 38 & 12.7 \\
\hline ROT8 & 37 & 200 & 62 & 6.4 & 26 & 201 & 51 & 9.1 \\
\hline SQR8 & 114 & 533 & 202 & 264.4 & 96 & 692 & 161 & 407.7 \\
\hline WGT8 & 58 & 261 & 64 & 60.5 & 22 & 120 & 29 & 64.3 \\
\hline
\end{tabular}

Table XI presents, for each function, the total number of cubes, the number of inputs to AND gates, the number of inputs to EXOR gates, and the user time. All these data are presented first for EXOR PLAs with 1-bit and next for 2-bit decoders. Table XII does the same for 3-bit decoders.

While Table XI and XII present the results on arithmetic functions, Table XIII shows the results on different kinds of functions from MCNC benchmarks. Three different cost functions are used for evaluating the results. $C_{T}$ measures the number of terms in the results. $C_{L}$ measures the number of literals in the results. $C$ is the cost function we introduced at page 31 . 
TABLE XII

EXPERIMENTAL RESULTS OF FUNCTIONS WITH 3 BIT DECODERS

\begin{tabular}{||l||l|l|l|l|}
\hline \multicolumn{1}{|c||}{} & \multicolumn{4}{c|}{ 3 bit decoder } \\
\cline { 2 - 5 } & cube & AND & EXOR & Time \\
\hline \hline ADR4 & 10 & 50 & 15 & 5.0 \\
\hline LOG8 & 41 & 307 & 112 & 544.2 \\
\hline MLP4 & 29 & 174 & 54 & 94.5 \\
\hline NRM4 & 26 & 170 & 46 & 73.3 \\
\hline RDM8 & 19 & 102 & 37 & 14.8 \\
\hline ROT8 & 16 & 102 & 26 & 11.6 \\
\hline SQR8 & 66 & 447 & 267 & 495.8 \\
\hline WGT8 & 10 & 78 & 17 & 65.9 \\
\hline
\end{tabular}

Figure 53 and 54 are scatter plots of number of terms versus execution time and number of variables versus execution time respectively. Both figures show week correlations between the independent variables and dependent variables. From these two figures we can see that the execution time depends more on the number of terms than on the number of variables. Since our basic algorithm is to perform cube operations iteratively, the execution time needed is mainly determined by the number of loops the program runs and the number of cubes in the array. In each loop, we check the difference for each pair of cubes. There are $(n \times(n-1)) / 2$ different pair of cubes in an array of $n$ cubes. So, if we go through one loop only, the time complexity is $O\left(n^{2}\right)$. Since we run the program for many loops, we can predict intuitively that the time complexity is higher than $O\left(n^{2}\right)$.

For analysis time complexity, we used multiple regression technique provide by a statistical package -- SPSS. The number of terms in the initial form and the number of variables in the form are taken as independent variables. The execution time is used as dependent variable. The regression generates the following results:

$$
\text { Execution Time }=-3.96+1.20 \times \operatorname{VARS}+2.32 \times\left(\frac{\text { TERM }}{100}\right)^{3}-0.11 \times e^{\left(\frac{T E R M}{100}\right)}
$$

where VARS is the number of variables in the form which equals to number of input vari- 
TABLE XIII

EXPERIMENTAL RESULTS OF EXORCISM-MV-2

\begin{tabular}{|c|c|c|c|c|c|c|c|c|}
\hline & \multirow{2}{*}{$\begin{array}{l}\text { input } \\
\text { var. }\end{array}$} & \multirow{2}{*}{$\begin{array}{l}\text { output } \\
\text { var. }\end{array}$} & \multicolumn{2}{|c|}{ INTTIAL FORM } & \multicolumn{4}{|c|}{ MINIMIZED FORM } \\
\hline & & & $C_{T}$ & $C_{L}$ & $C_{T}$ & $C_{L}$ & $C$ & Time \\
\hline हा2 & 15 & 9 & 57 & 358 & 28 & 163 & 28.46 & 2.0 \\
\hline bw & 5 & 28 & 26 & 369 & 22 & 320 & 22.87 & 2.1 \\
\hline clip & 9 & 5 & 163 & 1340 & 63 & 491 & 63.35 & 55.8 \\
\hline $\operatorname{con} 1$ & 7 & 2 & 10 & 44 & 9 & 37 & 9.84 & 0.1 \\
\hline duke2 & 22 & 29 & 103 & 1170 & 79 & 918 & 79.78 & 91.7 \\
\hline$e \times 5$ & 8 & 63 & 183 & 2739 & 78 & 860 & 78.31 & 61.1 \\
\hline inc & 7 & 9 & 34 & 229 & 28 & 174 & 28.76 & 3.5 \\
\hline misex 1 & 8 & 7 & 14 & 106 & 12 & 85 & 106.8 & 0.6 \\
\hline misex 2 & 25 & 18 & 28 & 217 & 27 & 210 & 27.97 & 3.5 \\
\hline $\mathrm{rd} 53$ & 5 & 3 & 31 & 183 & 15 & 68 & 15.37 & 0.3 \\
\hline rd73 & 7 & 3 & 127 & 999 & 39 & 192 & 39.19 & 9.8 \\
\hline rd84 & 8 & 4 & 255 & 2253 & 58 & 325 & 58.14 & 46.4 \\
\hline $\mathrm{saO} 2$ & 10 & 4 & 93 & 893 & 28 & 277 & 28.31 & 13.0 \\
\hline squar5 & 5 & 8 & 26 & 127 & 19 & 78 & 19.61 & 0.9 \\
\hline table 3 & 14 & 14 & 182 & 2767 & 166 & 2506 & 166.9 & 173.3 \\
\hline table 5 & 17 & 15 & 167 & 2687 & 156 & 2462 & 156.92 & 60.4 \\
\hline t481 & 16 & 1 & 980 & 13705 & 23 & 197 & 23.01 & 186.2 \\
\hline vg2 & 25 & 8 & 219 & 2570 & 184 & 2000 & 184.78 & 115.3 \\
\hline $5 \times \mathrm{xp} 1$ & 7 & 10 & 71 & 437 & 32 & 170 & 32.39 & 4.8 \\
\hline 9 sym & 9 & 1 & 145 & 1294 & 51 & 425 & 51.33 & 67.6 \\
\hline xor5 & 5 & 1 & 16 & 96 & 5 & 10 & 5.625 & 0.1 \\
\hline Adr4 & 8 & 5 & 155 & 1215 & 31 & 154 & 31.13 & 4.5 \\
\hline $\log 8$ & 8 & 8 & 408 & 3395 & 95 & 700 & 95.21 & 266.8 \\
\hline Mlp4 & 8 & 8 & 234 & 1909 & 62 & 387 & 62.20 & 38.2 \\
\hline Nrm4 & 8 & 5 & 313 & 2620 & 67 & 506 & 67.19 & 51.1 \\
\hline Rdm8 & 8 & 8 & 174 & 1295 & 31 & 152 & 31.12 & 8.5 \\
\hline Rot8 & 8 & 5 & 280 & 2355 & 37 & 262 & 37.11 & 6.4 \\
\hline Sqr8 & 8 & 16 & 505 & 4146 & 114 & 735 & 114.18 & 264.4 \\
\hline Wgt8 & 8 & 4 & 314 & 2757 & 58 & 325 & 58.12 & 60.5 \\
\hline
\end{tabular}

ables + number of output variables, and TERM is the number of terms in the initial form.

The above equation shows that the time complexity is $O\left(n^{3}\right)$ which is polynomial. Since we use the improvements of the current solution as termination criteria, if the current solution has been improved, the program will keep looping, otherwise it will stop. So, the execution time is not a deterministic one. The $\mathrm{R}$ square of the regression is 0.72 , which gives us a measurement of the randomness in the execution time. Detailed 


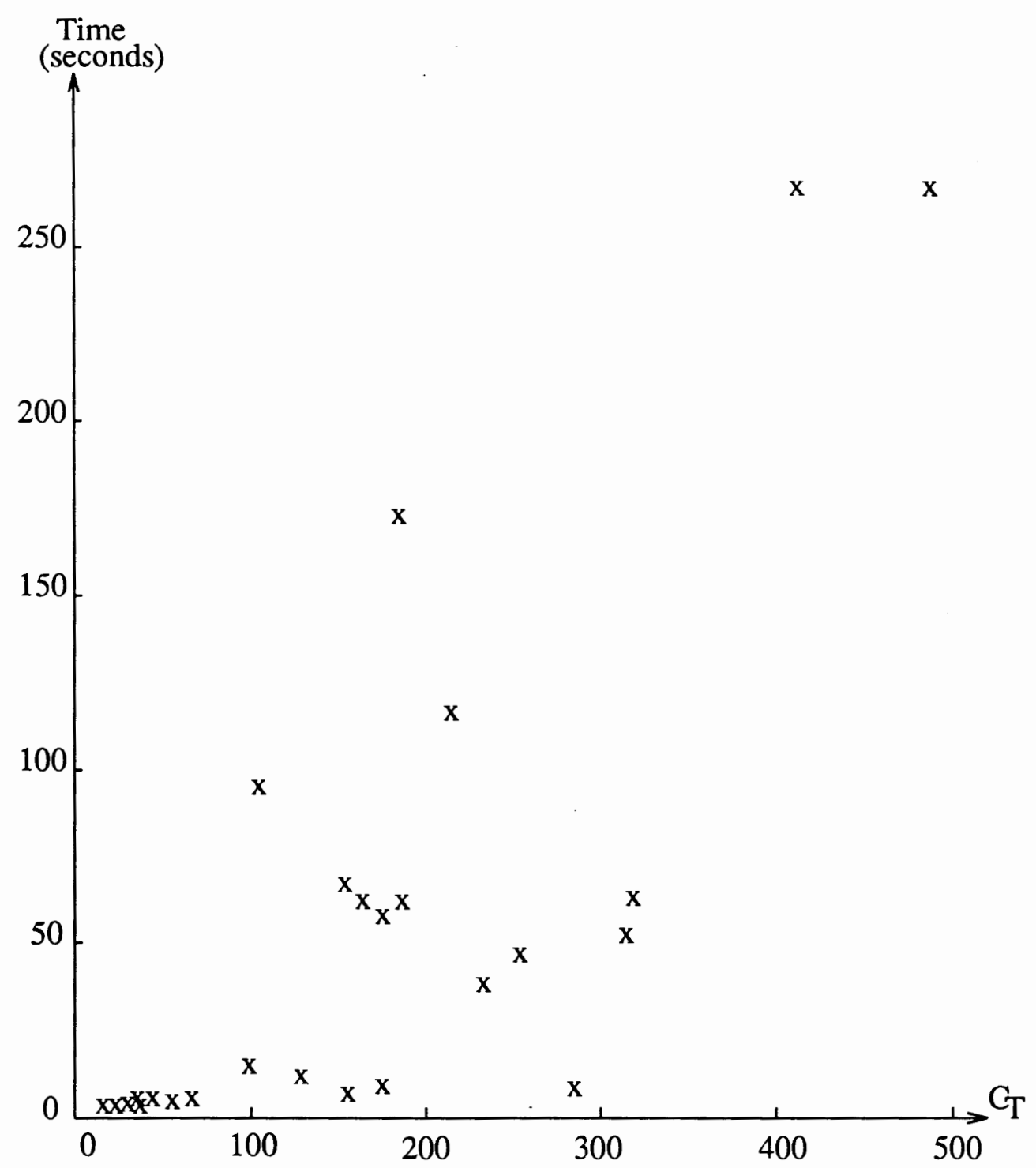

Figure 53. Scatter plot of number of terms versus execution time.

discussion on multiple regression can be found in any standard statistics books, for instance [Mend 87].

Table XIV and XV compare our results with those of EXMIN [Sasa 90a] and EXMIN-2 [Sasa 92] respectively. Since no timing information are provided in [Sasa 90a] and [Sasa 92] for these benchmarks, we can compare only cost functions in these two tables. While Table XIV compares $C_{T}$ only, Table XV compares all three cost functions. 


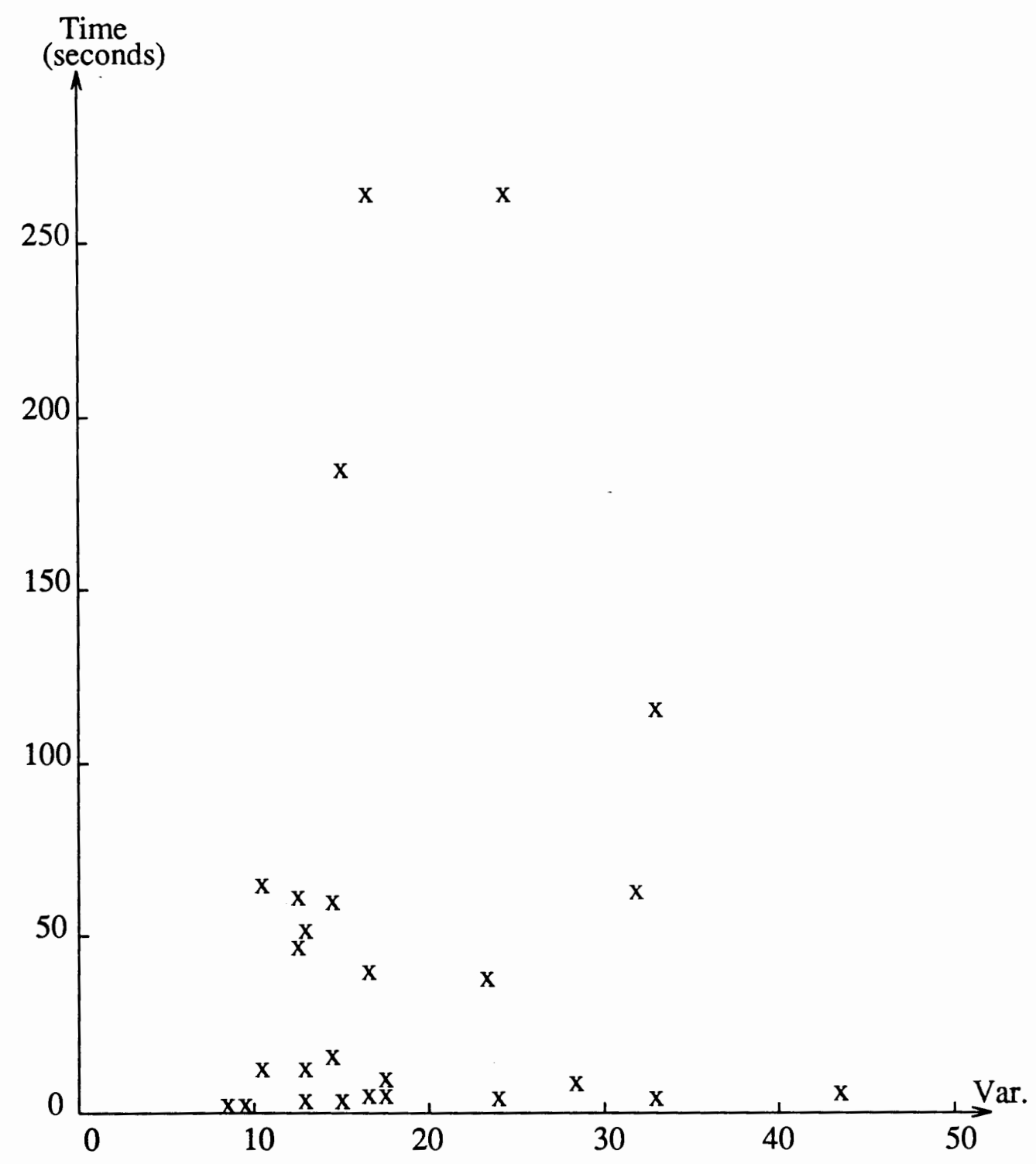

Figure 54. Scatter plot of number of variables versus execution time.

Table XVI and XVII compare other benchmarks with EXMIN-2. Both time and cost comparisons are shown in these two tables. Table XVI compares the execution time needed for achieving approximately the same cost functions $\left(C_{T}\right)$. At the end of each loop, the cost function is checked. The program will stop if the cost of the current solution is less than or equal to the target. We can see that in most cases our program generates the same or better results in shorter time. Table XVII compares the cost functions when the same time limits are given. Again, in most cases our program generates better 
TABLE XIV

COMPARISON OF EXORCISM-MV-2 WITH EXMIN

\begin{tabular}{||l||l|l||l|l|}
\hline \hline \multicolumn{1}{|c||}{} & \multicolumn{2}{c||}{ EXMIN } & \multicolumn{2}{|c|}{ EXORCISM-MV-2 } \\
\cline { 2 - 5 } & 1 bit & 2 bit & 1 bit & 2 bit \\
\hline \hline ADR4 & 32 & 11 & 31 & 11 \\
\hline LOG8 & 105 & 105 & 95 & 85 \\
\hline MLP4 & 66 & 56 & 62 & 51 \\
\hline NRM4 & 76 & 62 & 67 & 53 \\
\hline RDM8 & 31 & 28 & 31 & 26 \\
\hline ROT8 & 37 & 32 & 37 & 26 \\
\hline SQR8 & 121 & 121 & 114 & 96 \\
\hline WGT8 & 66 & 26 & 58 & 22 \\
\hline
\end{tabular}

TABLE XV

COMPARISON OF EXORCISM-MV-2 WITH EXMIN-2

\begin{tabular}{||l||l|l|l||l|l|l|l|}
\hline \hline \multicolumn{1}{|c||}{} & \multicolumn{3}{c||}{ EXMIN-2 } & \multicolumn{4}{c|}{ EXORCISM-MV-2 } \\
\cline { 2 - 8 } & $C_{T}$ & $C_{L}$ & $C$ & $C_{T}$ & $C_{L}$ & $C$ & Time \\
\hline \hline Adr4 & 31 & 162 & 31.13 & 31 & 154 & 31.13 & 4.5 \\
\hline Log8 & 99 & 690 & 99.20 & 95 & 700 & 95.21 & 266.8 \\
\hline Mlp4 & 63 & 395 & 63.21 & 62 & 387 & 62.20 & 38.2 \\
\hline Nrm4 & 71 & 533 & 71.20 & 67 & 506 & 67.19 & 51.1 \\
\hline Rdm8 & 32 & 161 & 32.12 & 31 & 152 & 31.12 & 8.5 \\
\hline Rot8 & 37 & 257 & 37.11 & 37 & 262 & 37.11 & 6.4 \\
\hline Sqr8 & 112 & 747 & 112.18 & 114 & 735 & 114.18 & 264.4 \\
\hline Wgt8 & 59 & 330 & 59.12 & 58 & 325 & 58.12 & 60.5 \\
\hline Adr4* & 11 & 60 & 11.05 & 11 & 60 & 11.05 & 4.7 \\
\hline Log8* & 92 & 944 & 92.28 & 85 & 826 & 85.24 & 500.3 \\
\hline Mlp4* & 50 & 412 & 50.22 & 51 & 455 & 51.24 & 89.6 \\
\hline Nrm4* & 52 & 521 & 52.20 & 53 & 515 & 53.20 & 66.8 \\
\hline Rdm8* & 26 & 181 & 26.14 & 26 & 190 & 26.15 & 12.7 \\
\hline Rot8* & 26 & 242 & 26.10 & 26 & 252 & 26.11 & 9.1 \\
\hline Sgr8* & 108 & 1078 & 108.26 & 96 & 853 & 96.21 & 407.7 \\
\hline Wgt8* & 25 & 159 & 25.06 & 22 & 149 & 22.05 & 64.3 \\
\hline
\end{tabular}

* with 2 bit decoders

results.

Table XVIII compares numbers of resultant terms for Espresso and Exorcism-mv-

2. Table XIX shows the experimental results for the minimization of incompletely 
TABLE XVI

COMPARISON OF EXORCISM-MV-2 WITH EXMIN-2

FOR THE SAME COST FUNCTIONS

\begin{tabular}{|c|c|c|c|c|c|c|c|c|}
\hline & \multicolumn{4}{|c|}{ EXMIN-2 } & \multicolumn{4}{|c|}{ EXORCISM-MV-2 } \\
\hline & $C_{T}$ & $C_{L}$ & $C$ & Time & $C_{T}$ & $C_{L}$ & $C$ & Time \\
\hline Бा2 & 28 & 164 & 28.46 & 4 & 28 & 163 & 28.46 & 3.5 \\
\hline clip & 68 & 517 & 68.39 & 55 & 66 & 490 & 66.37 & 49.2 \\
\hline rd53 & 15 & 60 & 15.33 & 2 & 15 & 60 & 15.33 & 1.2 \\
\hline $\mathrm{rd} 73$ & 42 & 221 & 42.22 & 20 & 40 & 207 & 40.21 & 8 \\
\hline rd84 & 59 & 330 & 59.15 & 45 & 59 & 338 & 59.15 & 69.3 \\
\hline $\mathrm{SaO} 2$ & 29 & 308 & 29.35 & 8 & 29 & 302 & 29.34 & 6.6 \\
\hline$t 481$ & 13 & 53 & 13.00 & 677 & 23 & 197 & 23.01 & 379.1 \\
\hline $\operatorname{vg} 2$ & 184 & 1992 & 184.78 & 163 & 184 & 2000 & 184.78 & 241.3 \\
\hline $5 \times 1$ & 34 & 186 & 34.43 & 13 & 33 & 190 & 33.43 & 1.7 \\
\hline 9 sym & 53 & 433 & 53.32 & 27.7 & 52 & 431 & 52.32 & 27.4 \\
\hline
\end{tabular}

TABLE XVII

COMPARISON OF EXORCISM-MV-2 WITH EXMIN-2 FOR THE SAME EXECUTION TIME

\begin{tabular}{|l||l|l|l|l||l|l|l|l|}
\hline \multicolumn{1}{|c||}{} & \multicolumn{3}{|c|}{ EXMIN-2 } & \multicolumn{1}{c|}{ EXOKCISM-MV-2 } \\
\cline { 2 - 9 } & $C_{T}$ & $C_{L}$ & C & Time & $C_{T}$ & $C_{L}$ & C & Time \\
\hline b12 & 28 & 164 & 28.46 & 4 & 28 & 163 & 28.46 & 3.5 \\
\hline clip & 68 & 517 & 68.39 & 55 & 64 & 485 & 64.36 & 55.2 \\
\hline rd53 & 15 & 60 & 15.33 & 2 & 15 & 60 & 15.33 & 1.2 \\
\hline rd73 & 42 & 221 & 42.22 & 20 & 39 & 192 & 39.19 & 19.8 \\
\hline rd84 & 59 & 330 & 59.15 & 45 & 62 & 351 & 62.16 & 45.5 \\
\hline sa02 & 29 & 308 & 29.35 & 8 & 29 & 294 & 29.33 & 7.4 \\
\hline t481 & 13 & 53 & 13.00 & 677 & 23 & 197 & 23.01 & 415 \\
\hline vg2 & 184 & 1992 & 184.78 & 163 & 184 & 2000 & 184.78 & 242.6 \\
\hline 5xp1 & 34 & 186 & 34.43 & 13 & 32 & 171 & 32.39 & 12.4 \\
\hline 9sym & 53 & 433 & 53.33 & 27.7 & 52 & 431 & 52.32 & 27.4 \\
\hline
\end{tabular}

specified functions. The results of minimizing $\mathrm{ON}$-cubes only are compared with the results of minimizing $\mathrm{ON}$ - and DC-cubes. The results show that better results are achieved when DC-cubes are taken into account. 
TABLE XVIII

COMPARISON OF OUR RESULTS WTTH ESPRESSO

\begin{tabular}{|c|c|c|c|c|c|c|c|}
\hline & \multirow{2}{*}{$\begin{array}{l}\text { input } \\
\text { var. }\end{array}$} & \multirow{2}{*}{$\begin{array}{l}\text { output } \\
\text { var. }\end{array}$} & \multirow{2}{*}{$\begin{array}{l}\text { ESPRESSO } \\
\text { cube }\end{array}$} & \multicolumn{4}{|c|}{ EXORCISM-MV-2 } \\
\hline & & & & cube & AND & EXOR & Time \\
\hline 612 & 15 & 9 & 43 & 28 & 123 & 40 & 2.0 \\
\hline bw & 5 & 28 & 22 & 22 & 81 & 239 & 2.1 \\
\hline clip & 9 & 5 & 120 & 66 & 380 & 111 & 32.8 \\
\hline con1 & 7 & 2 & 9 & 9 & 28 & 9 & 0.1 \\
\hline ex5 & 8 & 63 & 74 & 78 & 456 & 404 & 61.1 \\
\hline inc & 7 & 9 & 30 & 28 & 117 & 57 & 3.5 \\
\hline misex 1 & 8 & 7 & 12 & 12 & 48 & 37 & 0.6 \\
\hline misex 2 & 25 & 18 & 28 & 27 & 172 & 38 & 3.5 \\
\hline $\mathrm{rd} 53$ & 5 & 3 & 31 & 15 & 47 & 21 & 0.3 \\
\hline rd73 & 7 & 3 & 127 & 41 & 154 & 51 & 11.2 \\
\hline rd84 & 8 & 4 & 255 & 58 & 259 & 66 & 46.4 \\
\hline sao2 & 10 & 4 & 58 & 28 & 225 & 52 & 13.0 \\
\hline squar5 & 5 & 8 & 25 & 19 & 50 & 28 & 0.9 \\
\hline table3 & 14 & 14 & 175 & 166 & 1848 & 658 & 173.3 \\
\hline table5 & 17 & 15 & 158 & 156 & 1860 & 604 & 228.7 \\
\hline t481 & 16 & 1 & 481 & 23 & 174 & 23 & 201.0 \\
\hline vg2 & 25 & 8 & 110 & 184 & 1807 & 193 & 115.3 \\
\hline $5 \times \mathrm{xp} 1$ & 7 & 10 & 65 & 32 & 117 & 53 & 4.8 \\
\hline 9 sym & 9 & 1 & 86 & 51 & 374 & 51 & 67.6 \\
\hline xor5 & 5 & 1 & 16 & 5 & & 5 & 0.1 \\
\hline
\end{tabular}

TABLE XIX

COMPARISON OF MINIMIZATION OF ON-CUBES WITH MINIMIZATION OF ON- AND DC-CUBES

\begin{tabular}{||l||l|l|l|l||l|l|l|l||}
\hline \multicolumn{1}{|c||}{} & \multicolumn{3}{c||}{ ON-cubes } & \multicolumn{4}{c|}{ ON-andDC-cubes } \\
\cline { 2 - 9 } & cube & AND & EXOR & Time & cube & AND & EXOR & Time \\
\hline bench & 28 & 149 & 47 & 2.5 & 24 & 121 & 54 & 6.1 \\
\hline exam & 183 & 1467 & 353 & 284.9 & 160 & 1263 & 362 & 1504.1 \\
\hline p1 & 64 & 411 & 235 & 21.8 & 62 & 400 & 338 & 98.7 \\
\hline p3 & 40 & 262 & 119 & 10.6 & 39 & 252 & 143 & 51.5 \\
\hline test1 & 166 & 1056 & 448 & 330.6 & 164 & 1037 & 493 & 476.6 \\
\hline
\end{tabular}




\section{CHAPTER VII}

\section{CONCLUSION}

A new cube operation and algorithm for MIESOP minimization along with the efficient program to minimize such forms have been introduced. No algorithms have been published so far for MIESOP solutions to multi-output and incompletely specified functions.

One approach to minimize ESOPs is to apply a set of cube operations iteratively on each pair of cubes in the array. Our new operation -- exorlinking is the most general opertion in this approach which can link any two cubes in the array in arbitrary distance. All the cube operations introduced previously in the literature in this approach are the special cases of this operation.

Exorcism-mv-2 was tested on many benchmark functions and compared to EXMIN [Sasa 90a] and others. The program in most cases gives the same or better solutions on binary and 4-valued completely specified functions. More importantly, it is able to minimize efficiently arbitrary-valued and incompletely specified functions, while the programs from literature are only for completely specified functions: those from [Saul 90] and [Bess 91] only for binary variables, and the program from [Sasa 90a] for 2valued and 4-valued variables. Additionally, as in Espresso, the number of variables in our program is unlimited and the only constraint is the number of input cubes that are read, so very large functions can be minimized. 


\section{REFERENCES}

[Bess 83] Besslich, Ph.W., "Efficient Computer Method for EXOR Logic Design", Proc. IEE, Vol. 130, Part E, CDT, No. 6., pp. 203-206, 1983.

[Bess 91] Besslich, Ph. W., M.W. Riege, "An Efficient Program for Logic Synthesis of Mod-2 Sum Expressions", Euro ASIC'91, pp. 136-141, Paris, France, 1991.

[Bran 91] Brand, D., and T. Sasaso, "On the minimization of AND-EXOR expressions", 23rd IEEE Conference on Fault Tolerant Computing, pp. 1-9, 1990.

[Brow 90] Brown, F. M., "Boolean Reasoning", Kluwer Academic Publishers, 1990.

[CLi 91] Concurrent Logic, Inc., "CLi 6000 Series Field Programmable Gate Arrays. Preliminary Information", Dec. 1991, Rev. 1.3.

[Csan 92] Csanky, L., Perkowski, M., and I. Schaefer, "Canonical Restricted MixPolarity Exclusive Sums of Products and the Efficient Algorithm for Their Minimization", accepted for the publication in IEE proc. Pt. E., May, 1992.

[Flei 83] Fleisher, H., Tavel, M., and J. Yeager, "Exclusive-OR representations of Boolean functions", IBM J. Res. Develop., Vol. 27, pp. 412-416, July 1983.

[Flei 87] Fleisher, H., Tavel, M., and J. Yeager, "A Computer Algorithm for Minimizing Reed-Muller Canonical Forms", IEEE Trans. on Computers, Vol. C-36, No. 2, pp. 247 - 250, February 1987.

[FPGA 92] Proceedings of FPGA'92, 1992 ACM First International Workshop on Field-Programmable Gate Arrays, Hotel Durant, Berkeley, February 16-18, 1992.

[Froe 91] Froessl, J., and B. Eschermann, "Module Generation for AND/XOR-Fields (XPLAs)", Proc. IEEE ICCD-91 Conference, pp. 26-29, 1991.

[Fuji 86] Fujiwara, H., "Logic Testing and Design for Testability", Computer System Series, The MIT Press, 1986.

[Gr Grätzer, G., "Universal Algebra", 2nd edition,

[Gill 91] Gilliam, P., "A Practical Parallel Algorithm for the Minimization of Kroenecker Reed-Muller Expansions", M.S. Thesis, PSU EE Dept., 1991. 
[Gree 90] Green, D. H., "Reed-Mueller Canonical Forms with Mixed Polarity and Their Manipulations", IEE Proceedings, Vol. 137, Part E, No. 1, pp. 103113, January 1990.

[Gree 91] Green, D. H., "Families of Reed-Mueller canonical forms", International Journal of Electronics, pp. 259-280, February 1991, No. 2.

[Hell 88] Helliwell, M., and M.A. Perkowski:, "A Fast Algorithm to Minimize MultiOutput Mixed-Polarity Generalized Reed-Muller Forms", Proc. 25-th ACM/IEEE Design Automation Conference, pp. 427-432, June 12- June 15, 1988.

[Lui 92] Lui, P.K., and J.C. Muzio, "Boolean Matrix Transforms for the Minimization of Modulo-2 Canonical Expansions", IEEE Trans. on Computers, Vol. 41, No. 3, pp. 342-347, March 1992.

[Mend 87] Mendenhall, W., "Introduction to Probability and Statistics", 7th edtion, 1987, PWS Publishers.

[Papa 79] Papakonstantinou, G., "Minimization of modulo-2 sum of products", IEEE Trans. on Computers., Vol. C-28, pp. 163-167, February 1979.

[Perk 89] Perkowski, M., Helliwell, M., and P. Wu, "Minimization of multiple-valued input multi-output mixed-radix exclusive sums of products for incompletely specified boolean functions", Proc. of the 19th International Symposium on Multiple-valued Logic, pp. 256-263, May 1989.

[Perk 90] Perkowski, M., and M. Chrzanowska-Jeske, "An Exact Algorithm to Minimize Mixed-Radix Exclusive Sums of Products for Incompletely Specified Boolean Functions", Proc. of the ISCAS'90, International Symposium on Circuits and Systems, pp. 1652-1655, New Orleans, May 1990.

[Perk 91] Perkowski, M., and P. Johnson, "Canonical Multi-Valued Input ReedMueller Trees and Forms", Proc. of 3rd NASA Symposium on VLSI Design, pp. 11.3.1 - 11.3.13, University of Idaho, Oct 30-31, 1991.

[Perk 92] Perkowski, M., "Generalized Orthonormal Expansion and Some of Its Applications", Proc. Intern. Symp. on Multiple-Valued Logic, pp. 442 - 450, Sendai, Japan, May 1992.

[Prad 87] Pradhan, D.K., "Fault-Tolerant Computing. Theory and Techniques. Vol. I." Prentice-Hall, 1987.

[Robi 82] Robinson, J.P., and C.L. Yeh, "A Method for modulo-2 Minimization", IEEE Trans. on Computers, Vol. C-31, pp. 800-801, August 1982.

[Rude 85] Rudell, R.L., and A.L. Sangiovanni-Vincentelli, "ESPRESSO-MV: algorithms for multiple-valued logic minimization, Proc. IEEE Custom Integrated Circuits Conf., 1985. 
[Salm 89] Salmon, J. V., E.P. Pitty, E.P., and Abramson, M. S., "Syntactic Translation and Logic Synthesis in Gatemap", IEE Proceedings, Vol. 136, Part E., No. 4, pp. 321-328, July 1989.

[Sara 92] Sarabi, A., and M.A. Perkowski, "Fast Exact and Quasi-Minimal Minimization of Highly Testable Fixed-Polarity AND/XOR Canonical Networks", Proc. 1992 IEEE Design Automation Conference, pp. 30 - 35, June 1992.

[Sasa 78] T. Sasao, "An application of multiple-valued logic to a design of Programmable Logic Arrays", Proc. 8th Intern. Symp. on Multiple-Valued Logic (ISMVL), pp. 65 - 72, May 1978.

[Sasa 81] Sasao, T., "Multiple-valued decomposition of generalized boolean functions and the complexity of programmable logic arrays," IEEE Trans. Comput., Vol. C-30, pp. 635-643, Sept. 1981.

[Sasa 84] Sasao, T., "Input Variable Assignment and Output Phase Optimization of PLA's", IEEE Trans. on Comp., Vol. C-33, No. 10, pp. 879-894, October 1984.

[Sasa 86] Sasao, T., "MACDAS: Multi-level AND-OR circuit synthesis using twovariable genrators", Proc. of 23-rd Design Automation Conference, Las Vegas, pp. 86-93, June 1986.

[Sasa 90a] Sasao, T., "EXMIN: A simplification algorithm for Exclusive-OR-Sum-ofProducts expressions for multiple-valued input two-valued output functions", Proc. ISMVL-90, May 1990, pp.128-135.

[Sasa 90b] Sasao, T., and Besslich, Ph., "On the Complexity of MOD-2 Sum PLAs", IEEE Transactions on Computers, Vol. 39., No. 2, pp. 262-266, February 1990.

[Sasa 91a] Sasao, T., "A transformation of multiple-valued input two-valued output functions and its application to simplification of exclusive-or sum-ofproducts expressions", Proc. ISMVL-91, May 1991, pp. 270-279.

[Sasa 91b] Sasao, T., "On the complexity of some classes of AND-EXOR expressions", IEICE Technical Report FTS 91-35, October 1991.

[Sasa 92] Sasao, T., private communication between Dr. Sasao and Dr. Perkowski.

[Saul 90] Saul, J. M., "An Improved Algorithm for the Minimization of Mixed Polarity Reed-Mueller Representations", Proc. Intern. Conf. on Computer Design: VLSI in Computers and Processors, pp. 372 - 375, September 17-19, 1990,

[Saul 91] Saul, J. M., "An Algorithm for the Multi-level Minimization of Reed-Muller Representations", Proc. Intern. Conf. on Computer Design: VLSI in Computers and Processors, 1991, pp. 634-637. 
[Scha 91] Schaefer, I., and M. Perkowski, "Multiple-Valued Input Generalized ReedMuller Forms", Proc. of ISMVL'91, pp. 40-48, May 1991.

[Scha 92] Schaefer, I., and M. Perkowski, "Exact Minimization of Multiple-Valued Input Kronecker Reed-Muller Forms for Incompletely Specified Functions", Submitted to IEEE Transactions on Computers, April 1992.

[Stol 79] stoll, R. R., "Set Theory and Logic", Dover Publications, Inc. New York, 1979.

[Wu 82] Wu, X., Chen, X., and Hurst, S.L., "Mapping of Reed-Muller codfficients and the minimisation of exclusive OR-switching functions", IEE proc. E, Comput. \& Digital Tech., 1982, pp. 15-20.

[Wu 92] Wu, L-F., Perkowski, M., "Exact and Approximate Minimization of ReedMuller Trees for Cellular Logic with Application to CLI6000 Field Programmable Gate Arrays", Proc. Second International Workshop on FieldProgrammable Logic and Applications, Vienna, Austria, Aug. 31 - Sept. 2, 1992. 


\section{APPENDIX \\ MAN PAGE OF EXORCISM-MV-2}

NAME

EXORCISM-MV-2 -- EXOR CIrcuit Speedy Minimizer, for Multiple-valued logic, Version II.

SYNOPSIS

exorcism [inputfile][options].

The inputfile is an ascii file containing data of a given logic function. The inputfile should be prepared before hand. To minimize the logic function, type exorcism inputfile.

The optimized solution will be shown on the screen. For saving the solution in a file, type exorcism inputfile > outputfile

where outputfile is the output file name given by the user. DESCRIPTION

Exorcism-MV-2 takes an ESOP or a disjoint SOP as input. The function can be binary input or multiple-valued input, single output or multiple output, completely or incompletely specified. If the function is a multiple output function, the algorithm will translate it to a multiple-valued input description. If the function is incompletely specified, the program will separate it to an array of ON-cubes and an array of DC-cubes. The final result will be a minimal or near minimal logically equivalent set of product terms to represent the $\mathrm{ON}$-array and optionally terms which lies in the DC-array. 
Comments are allowed within the input by placing a pound sign "\#" as the first character on a line. Comments and unrecognized keywords are passed directly from the input file to standard output. Any white space (blanks, tabs, etc), except when used as a delimiter in an embedded command, is ignored.

\section{KEYWORDS}

The following keywords can be shown in the inputfile. [d] denotes a decimal number and [s] denotes a text string.

.i [d]

Specifies the number of input variables, if the function is binary input and binary output.

$.0[d]$

Specifies the number of output variables, if the function is binary input and binary output.

.pair [d]

Specifies the number of pairs of variables which will be paired together by using two-bit decoders. The rest of the line contains pairs of numbers which specify the binary variables of the XPLA which will be paired together. The XPLA will be reshaped so that any unpaired binary variables occupy the leftmost part of the array, then the paired multiple-valued columns, and finally any multiple-valued variables.

.$m v[d][d][d] \ldots[d]$

The first $d$ specifies the number of variables for multiple-valued functions. The second $d$ shows the number of binary input variables. The rest of the line gives information of each multiple-valued variables.

.$p[d]$

Specifies the number of product terms. 
.e (.end)

Marks the end of the input description.

\section{EXAMPLE 1:}

The first example is shown a description of a function with 4 binary input variables and 1 binary output variable. a - indicates the output is don't care corresponding to the input combination.

$$
\begin{aligned}
& . \mathrm{i} 4 \\
& .01 \\
& . p 7 \\
& 00011 \\
& 01011 \\
& 11001 \\
& 11111 \\
& 1110- \\
& 1001- \\
& 0011- \\
& . e
\end{aligned}
$$

\section{EXAMPLE 2:}

This example shows a description of a multiple-valued function with no binary variable and 3 multiple-valued variables, where the multiple-valued variables have sizes of 4,4 , and 3 , and the last multiple-valued variable is the "output".

.mv 30443

.p 3

11011101101

01010010110 
.$e$

\section{OPTIONS}

The command line options can be specified anywhere on the command line and must be separated by spaces.

-help

Provides a quick summary of the available command line options.

$-\mathbf{s}$

provides a short summary of the execution of the program including the initial cost of the function, the final cost, and the computer resources used.

EXAMPLE 3:

Given an input file test6, type

exorcism test6 -s

the output will be:

\# Portland State University, EXORCISM-MV Version \#2, Release date 5/18/92

\# XPLA is test 6 with 5 inputs and 1 outputs

\# ON-set cost is $c=31(31)$ in $=139$ out $=31$ tot $=170$

\# OFF-set cost is $c=1(1)$ in $=5$ out $=1$ tot $=6$

\# DC-set cost is $c=0(0)$ in $=0$ out $=0$ tot $=0$

\# $0 \mathrm{~K}$ bytes active out of $1 \mathrm{~K}$ bytes allocated

\# EXORCISM Time was $0.1 \mathrm{sec}$, cost is $\mathrm{c}=8(8)$ in $=22$ out $=8$ tot $=30$

i 5

.01

.p 8

$-0001$

111111

$-0-1$ 
$01-01$

$1-11$

$001-1$

$10-0-1$

$-1-1-1$

.$e$

$-\mathbf{t}$

Provides a trace showing the execution of the program. After each step of the algorithm a single line is printed which reports the processor time used, and the current cost of the function.

$-\mathbf{x}$

Suppresses printing of the solution.

EXAMPLE 4:

If we type

\section{exorcism test6 $-\mathrm{x}-\mathrm{s}$}

the output will be:

\# Portland State University, EXORCISM-MV Version \#2, Release date 5/18/92

\# XPLA is test6 with 5 inputs and 1 outputs

\# ON-set cost is $c=31(31)$ in $=139$ out $=31$ tot $=170$

\# OFF-set cost is $c=1(1)$ in $=5$ out $=1$ tot $=6$

\# DC-set cost is $c=0(0)$ in $=0$ out $=0$ tot $=0$

\# $0 \mathrm{~K}$ bytes active out of $1 \mathrm{~K}$ bytes allocated

\# EXORCISM Time was $0.1 \mathrm{sec}$, cost is $\mathrm{c}=8(8)$ in $=22$ out $=8$ tot $=30$

- mo

Select the option "minimize the multiple output directly". The multiple output function will not be decomposed to a set of single output functions 
if this option is selected.

$-d c$

Select the option "don't care". If this option is selected, the program will minimize the ON-array with the DC-array. Otherwise, only the ON-array will be minimized.

- do $[s]$

This option executes subprogram. [s] denotes a name of subprogram. Some useful subprograms are listed separately below:

-do echo

This echoes "-out fdr" to standard output, and to compute the complement of a function.

-do stats

Provide simple statistics on the size of the function.

\section{EXAMPLE 5:}

If we type

\section{exorcism test6 -do stats}

we will get the following output:

\# Portland State University, EXORCISM-MV Version \#2, Release date 5/18/92

\# XPLA is test6 with 5 inputs and 1 outputs

\# ON-set cost is $c=31(31)$ in $=139$ out $=31$ tot $=170$

$\#$ OFF-set cost is $c=0(0)$ in $=0$ out $=0$ tot $=0$

\# DC-set cost is $c=0(0)$ in $=0$ out $=0$ tot $=0$

If we use this option with the option $-s$, this option will be ignored. 